\title{
The neurocognitive underpinnings of the Simon effect: An integrative review of current research
}

\author{
Jesús Cespón ${ }^{1}$ • Bernhard Hommel ${ }^{2}$ - Margarethe Korsch $^{3} \cdot$ Daniela Galashan $^{3}$ \\ Accepted: 13 September 2020 / Published online: 7 October 2020 \\ (C) The Psychonomic Society, Inc. 2020, corrected publication 2020
}

\begin{abstract}
For as long as half a century the Simon task - in which participants respond to a nonspatial stimulus feature while ignoring its position - has represented a very popular tool to study a variety of cognitive functions, such as attention, cognitive control, and response preparation processes. In particular, the task generates two theoretically interesting effects: the Simon effect proper and the sequential modulations of this effect. In the present study, we review the main theoretical explanations of both kinds of effects and the available neuroscientific studies that investigated the neural underpinnings of the cognitive processes underlying the Simon effect proper and its sequential modulation using electroencephalogram (EEG) and event-related brain potentials (ERP), transcranial magnetic stimulation (TMS), and functional magnetic resonance imaging (fMRI). Then, we relate the neurophysiological findings to the main theoretical accounts and evaluate their validity and empirical plausibility, including general implications related to processing interference and cognitive control. Overall, neurophysiological research supports claims that stimulus location triggers the creation of a spatial code, which activates a spatially compatible response that, in incompatible conditions, interferes with the response based on the task instructions. Integration of stimulus-response features plays a major role in the occurrence of the Simon effect (which is manifested in the selection of the response) and its modulation by sequential congruency effects. Additional neural mechanisms are involved in supporting the correct and inhibiting the incorrect response.
\end{abstract}

Keywords Simon task - Event-related potentials · Electroencephalogram · Transcranial magnetic stimulation · Functional magnetic resonance imaging $\cdot$ Sequential congruency effects

\section{Introduction and scope of review}

The Simon task (Simon \& Small, 1969) represents - even more than the notorious Stroop task (Stroop, 1935; MacLeod, 1991) and the Flanker task (Eriksen \& Eriksen, 1974) - the signature tool to study stimulus-response compatibility (SRC) (Hommel, 2011a; Kornblum, Hasbroucq \&

Electronic supplementary material The online version of this article (https://doi.org/10.3758/s13415-020-00836-y) contains supplementary material, which is available to authorized users.

Jesús Cespón

jesuscespon@gmail.com

1 Basque Center on Cognition, Brain and Language, Mikeletegi Pasealekua, 69, 20009 Donostia/San Sebastián, Spain

2 Leiden Institute for Brain and Cognition and Leiden University Institute of Psychology, Leiden, The Netherlands

3 Department of Neuropsychology and Behavioral Neurobiology, University of Bremen, Bremen, Germany
Osman, 1990) and it is widely used to study cognitive functions in cognitive psychology and neuroscience, such as visuospatial attention, cognitive control, and motor planning. In a typical Simon task, participants respond to a non-spatial feature (e.g., colour, shape) of a lateralised stimulus by pressing one of two horizontally organised response buttons, so that each response button corresponds to one stimulus position. Although the stimulus position is irrelevant to performing the task, the reaction time (RT) is longer when the response location does not correspond to (is spatially incompatible with) the stimulus location (incompatible condition) than when it does (compatible condition). This spatial interference, known as Simon effect, is obtained with visual (Craft \& Simon, 1970), auditory (Simon \& Small, 1969), and somatosensory (Hasbroucq \& Guiard, 1992) stimulation, regardless of whether the participants respond by using hand, foot or eye movements (Leuthold \& Schröter, 2006). Some typical Simon task paradigms are shown in Fig. 1.1. In contrast to Stroop and Flanker effects, which reflect a mixture of stimulus-response (S-R) and stimulus-stimulus (S-S) conflicts (De Houwer, 2003; Fournier, Scheffers, Coles, Adamson, \& Villa Abad, 


\subsection{Representation of typical Simon tasks}

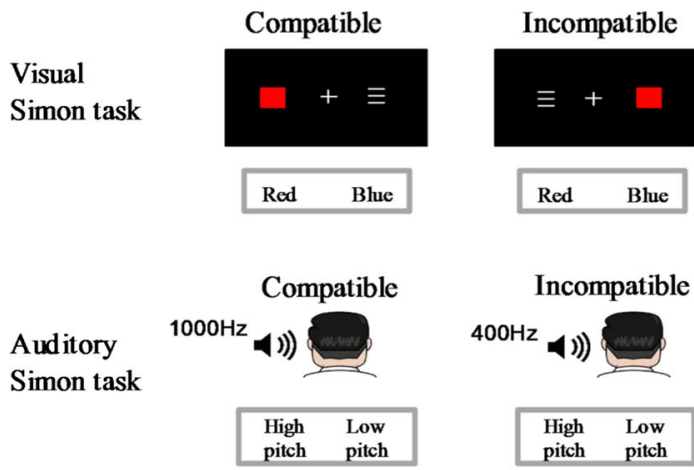

\subsection{Speed of processing and distributional analysis}
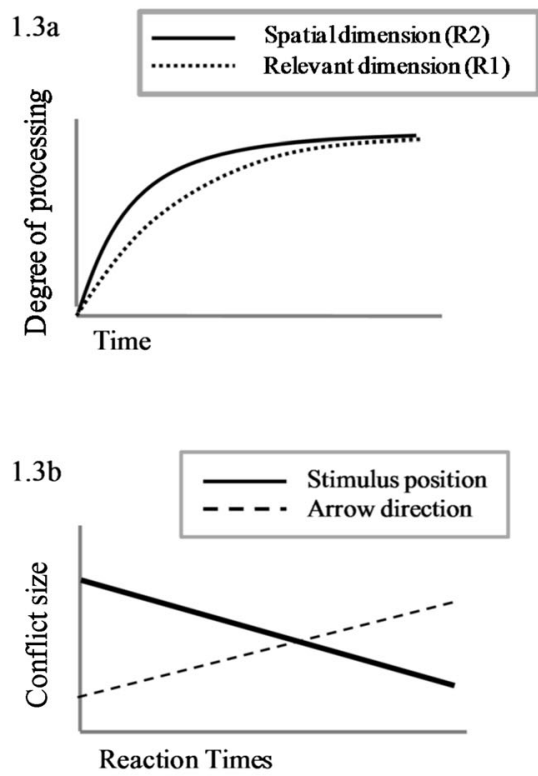

\subsection{General structure of dual route models}

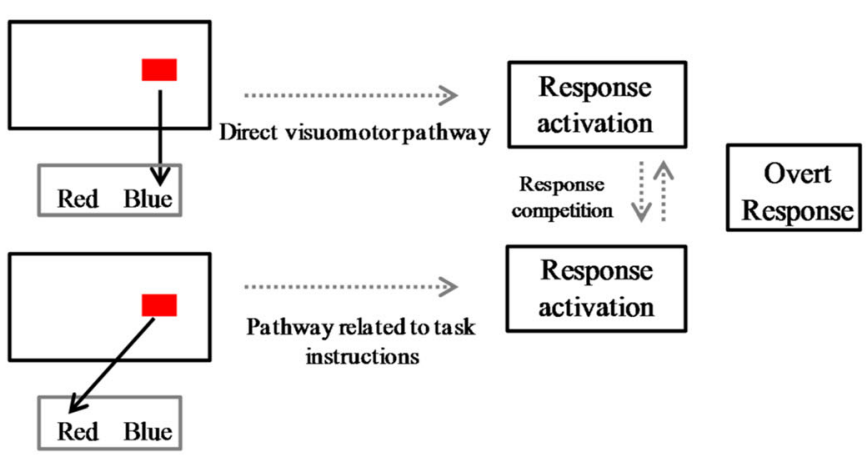

\subsection{Theoretical mechanisms to select the correct response}

Top-down cognitive control

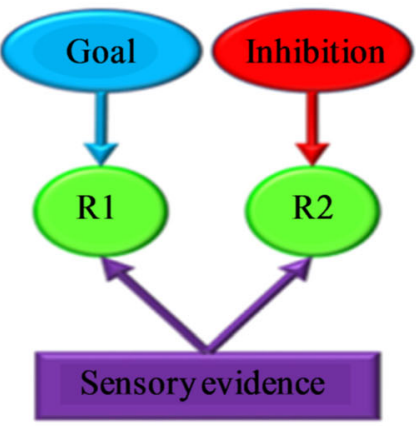

\section{Reciprocal inhibition}

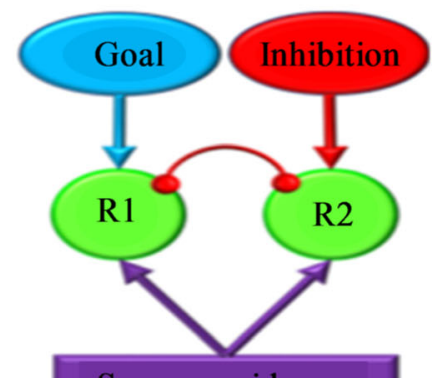

Sensory evidence

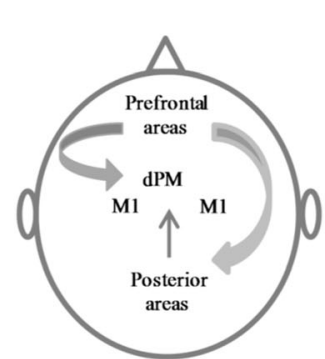

Involved brain areas

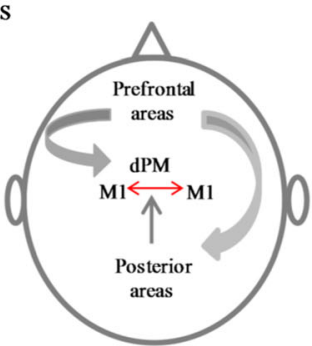

Fig. 1 Mechanisms related to interference and conflict resolution occurring during Simon task performance. Representation of typical visual and auditory Simon tasks (1.1). Dual route models (1.2) postulated differences in speed (1.3a) and strength (1.3b) of the competing responses as mechanisms giving rise to the Simon effect.

Distributional analysis of the reaction times suggested that speed of processing of the irrelevant dimension modulates the temporal dynamic of the interference (1.3c). Feedforward and reciprocal inhibition were proposed as mechanisms involved in resolving the spatial conflict occurring during the Simon task (1.4)

1997), the Simon effect can be unambiguously attributed to SR conflict (Kornblum, Hasbroucq, \& Osman, 1990). This simplicity and the large number of processes that it allows studying can explain the Simon task's growing popularity over the last decades (Hommel, 2011a).

In the following sections, we briefly outline the main theoretical accounts used to explain different aspects of the cognitive processes taking place in the Simon task, with a main focus on the cognitive control of the interference and its modulation by contextual factors (e.g., sequential congruency effects). In general, these theoretical accounts are limited since they were developed on the basis of the overt performance. For this reason, neurophysiological studies using electroencephalogram (EEG), event-related potentials (ERP), transcranial magnetic stimulation (TMS), and functional magnetic resonance imaging (fMRI) were conducted to obtain information in high temporal (EEG/ERP, TMS) and spatial (fMRI) resolution about neural activity occurring during the performance of Simon-type tasks. For each neurophysiological subsection, we briefly introduce the technique and then discuss to 
what extent the available research sheds light on several key theoretical issues (a table of contents of the manuscript is provided in Supplementary Information_S1 (Online Supplementary Material)). We performed a comprehensive review of all studies providing significant insights with respect to the theoretical issues that are formulated in the next section. Overall, all the relevant EEG/ERP and TMS studies, i.e., those studies using Simon tasks and analysing compatible and incompatible conditions and/or sequential congruency effects in healthy young subjects, were included in this paper. It is pertinent to state that we have mainly (although not exclusively) focused on visual Simon tasks with manual responses since it was, by far, the more used variant of the task. Also, for fMRI, we use information from a meta-analysis (appended as Supplementary Information), a label-based analysis, and some specific studies to evaluate the plausibility of specific theoretical accounts. Given the multidimensional nature of fMRI data (i.e., the fact that several thousand of voxels may be activated on each study), the meta-analysis represents an optimal strategy to reveal the main areas activated during conflict processing and interference resolution across all aggregated studies. Specific inclusion criteria for fMRI meta-analysis are provided in the corresponding section.

\section{Theoretical accounts and key questions}

The Simon effect itself and its modulation by contextual factors have stimulated various theoretical efforts. It is important to emphasise that not all theoretical models that are related to the Simon effect intend to explain all aspects of the effect. We first discuss those that actually do, and we see that they all assume that, in one way or another, the code of the relevant stimulus feature (e.g., colour or shape) or the corresponding response is involved in a conflict with the code of the irrelevant spatial stimulus feature or of the corresponding response. The key difference is that some approaches locate this conflict earlier in the processing chain than others. We refer to the models that aim to explain the Simon effect proper as Simon-effect models.

Other theoretical models do not aim to explain the effect itself but rather ask how and under what conditions the stimulus and/or response codes involved in the conflict are generated. Given that in the Simon task it is the spatial code that matters most, we refer to the models aiming to explain the generation of these codes as spatial-coding models. Similarly, some models have focused on the temporal relationship between the conflicting codes and asked whether and to what degree - the size and occurrence of the Simon effect are determined by this relationship. We refer to these models as time-difference models.

Even other theoretical models do not aim to explain the Simon effect proper or particular coding processes, but rather focus on the finding that the size of the Simon effect is systematically modulated by the compatibility of the previous trial (see below). As we discuss, there are two kinds of models that are not mutually exclusive and may even conceptually overlap. We refer to these models as sequential-effect models.

In the following, we briefly sketch the basic ingredients and assumptions of all four kinds of models and then extract six theoretical implications of the available models that neuroscientific methods are particularly suitable to test. These six implications or key questions serve to structure the neurophysiological findings.

\section{Simon-effect models}

\section{Dual-route models}

The Simon task represents one of the main experimental paradigms to study how irrelevant information interferes with the intended behaviour. Theoretical explanations of the Simon effect were frequently based on dual-route models (see Fig. 1.2), which attribute the Simon effect to a competition between two alternative responses (De Jong, Liang, \& Lauber, 1994; Kornblum, Hasbroucq, \& Osman, 1990; Proctor \& Vu, 2006; Wascher, Schatz, Kuder, \& Verleger, 2001). In general, dual-route models argue that the irrelevant dimension (i.e., the stimulus location) activates a spatially corresponding/ compatible response through a fast and direct route - even though most available models are not very articulate regarding the reason why this route is fast and direct (Hommel, 2011b). If this response is not the one that is demanded by task instructions, which is activated through a slower, controlled, and indirect route, its activation delays the execution of the correct response. Thus, in incompatible trials, the selection of the correct response is delayed (represented in Fig. 1.2) due to the time required to resolve the response conflict created by the irrelevant stimulus location and the respondents it activates (see also Fig. 1.3a).

Dual-route models have two important theoretical implications. First, they imply that more than one response representation can become active at a time, which in one way or another creates a conflict that needs to be resolved before response selection can be completed. Second, the resolution of this conflict must rely on some sort of top-down support for the correct response. Taking into account that stimulus location is processed earlier than the relevant feature of the stimulus (as suggested by time-difference models described below), a response-selection mechanism operating on a first-in/ first-shoot principle would produce too many errors in incompatible trials to be realistic. Accordingly, what is needed is a mechanism that makes sure that conflict resolution proceeds in such a way that the correct response is likely to win in the end. While these two assumptions are shared by all dual-route 
models, they differ with respect to a third assumption, which relates to the question whether concurrently active response representation does or does not interact with each other (both possibilities are represented in Fig. 1.4).

Some models assume that, in incompatible trials, the representation of the correct response and the representation of the location-induced incorrect response directly compete with each other, so that activating one representation leads to the lateral inhibition of the other (e.g., Zorzi \& Umiltà, 1995). While top-down support will help the representation of the correct response to eventually outcompete the representation of the incorrect one, the reciprocal inhibition between the two representations will slow down the build-up of the activation of the correct response representation (Bogacz, 2007; Bogacz, Brown, Moehlis, Homes, \& Cohen, 2006). Other models do not assume direct interactions between alternative response representations. That is, concurrent activation (in incompatible trials) is also assumed to create conflict but the resolution of this conflict is exclusively based on top-down control mechanisms, as assumed, for instance, by diffusion models for conflict tasks (Servant, White, Montagnini, \& Burle, 2016; Ulrich, Schröter, Leuthold, \& Birngruber, 2015).

\section{Top-down inhibition models}

Some dual-route models assume that the resolution of the response conflict is achieved through some kind of interaction between concurrently active codes and a top-down control system that supports the correct response (De Jong, Liang, \& Lauber, 1994), but other dual-route models have claimed the existence of another instance that serves to inhibit the representation of incorrect responses (Kornblum, Hasbroucq, \& Osman, 1990), such as the activation-suppression model (Ridderinkhof, 2002). On the one hand, these models are obviously less parsimonious than the standard version of the dual-route model, as they postulate an additional system with a function that may just as well be covered by the top-down support system that needs to be assumed anyway. On the other hand, however, proponents of top-down inhibition models have provided supporting evidence from analyses of RT distributions.

It is known that the Simon effect is stronger for fast responses than it is for slow responses. While earlier approaches have attributed this effect to the spontaneous decay of spatial information (De Jong, Liang, \& Lauber, 1994; Hommel, 1993), not unlike in iconic memory, and even though evidence for the spontaneity of this decay was reported (e.g., Hommel, 1994), the activation-suppression model (Ridderinkhof, 2002) has attributed the temporal dynamics to active inhibition. In particular, the model argues that active suppression of conflicting responses requires time to be effective and is implemented only after processing the irrelevant dimension. Predictions from this model are also consistent with distributional analyses of RTs (De Jong, Liang, \& Lauber 1994; Dittrich, Kellen, \& Stahl, 2014; Proctor, Miles, \& Baroni, 2011), but the interpretation differs: the finding that the effect size decreases with increasing RT is not taken to reflect spontaneous decay but active inhibition of the incorrect response through cognitive control processes (Fig. 1.3b).

Studies inducing spatial conflict by using other irrelevant dimensions, such as the pointing direction of a central arrow, showed that interference is smaller or absent at fast RTs and increases at slow RTs (Fig. 1.3b) (Cespón, Galdo-Álvarez, \& Díaz, 2013a; Pellicano, Lugli, Baroni, \& Nicoletti, 2009; Proctor, Miles, \& Baroni, 2011). This is in line with findings suggesting that arrows are less powerful in orienting spatial attention than spatial location (Abrahamse \& Van der Lubbe, 2008; Klein \& Ivanoff, 2011). Several studies suggested that these findings could be related to the time required to interpret the symbolic content of the arrow (Iani, Ricci, Baroni, \& Rubichi, 2009; Symes, Ellis, \& Tucker, 2005; Vainio, Ellis, $\&$ Tucker, 2007). According to the activation-suppression model, conflict from arrow direction is absent at faster RTs because the response triggered by this irrelevant dimension is not yet accessible to the response system and thus cannot interfere with the response to the relevant dimension. Also, studies showed increased interference effect at slowed RTs when placing the stimuli in a vertical arrangement (Proctor, $\mathrm{Vu}, \&$ Nicoletti, 2003; Wiegand \& Wascher, 2005). Even if these findings suggest evidence against spontaneous-decays models, they may be also interpreted as a result of slower processing of the stimulus location in vertical than horizontal Simon tasks. Crucially, both spontaneous-decay models and the activation-suppression model are in line with studies suggesting that the time required to process the relevant and irrelevant dimensions largely determines the time course of the conflict (Hommel, 1993, 1994; Ulrich, Schröter, Leuthold, \& Birngruber, 2015) - an issue we return to in the section about time-difference models.

\section{Perceptual models}

While the bulk of the available models can be categorised as dual-route models, the implication that the Simon effect reflects the activation of multiple responses is not shared by all models. In particular, Hasbroucq and Guiard (1991) have suggested that the Simon effect might reflect stimulus incongruity. The idea is that, if a non-spatial stimulus feature, like with red and green colour dots, is used to indicate the correct response, the representations of these features acquire the meaning corresponding to the spatial location of the response. If, say, a red dot signals a left response, the red stimulus acquires the meaning "left", which in turn would conflict with perceiving the stimulus on the right side. In other words, the Simon effect might reflect a conflict between the location and the 
meaning of stimuli, and the time needed to resolve this conflict. While the conflict idea is shared by dual-route models, the stimulus-congruency model does not imply multiple response activation, response conflict, or response-conflict resolution, which leads to very different predictions regarding neural measures.

\section{Spatial-coding models}

The Simon task paradigm has also stimulated theoretical developments regarding the relationship between attention and action. Actually, attentional accounts of the Simon effect were suggested since the earliest studies. Simon and Small (1969) related the Simon effect to a primitive tendency to react towards the source of stimulation, which would represent a kind of orienting reflex (Sokolov, 1963). Subsequently, a large number of studies focused on explaining how and when a given stimulus is spatially coded and whether or how attention towards a lateralised stimulus interferes with responding to a task-relevant non-spatial feature (e.g., colour, shape) of it.

The attentional shift account was used to explain how and why a stimulus is spatially coded as left or right so that it directly triggers a spatially compatible response (Nicoletti \& Umiltà, 1994). This explanation states that, during the performance of a Simon task, the stimulus is initially detected without processing its spatial location. Then, an attentional shift is executed towards the stimulus location and the corresponding spatial code is formed. Next, the non-spatial features of the stimulus are processed and, if required, a response is prepared. This theory, schematically depicted in Fig. 2.1, represents an "early-attention" account as non-spatial stimulus features can only be processed after attention has been allocated to the location of the stimulus.

Unfortunately, the attentional shift account does not propose a specific mechanism to explain why spatial selection results in activating the spatially compatible response. However, the account does refer to the premotor theory of attention (PMTA) to provide such an explanation (Rizzolatti, Riggio, Dascola, \& Umiltà, 1987; Sheliga, Craighero, Riggio, \& Rizzolatti, 1997). The PMTA argues that an attentional shift is driven by a saccadic motor program, which the attentional account assumes to interact with other motor programs as well. And yet, in contrast to the attentional shift account, the PMTA states that the attentional shift and the associated motor program occur after processing all stimulus features. In this sense, the PMTA represents a "late-attention" account of the Simon effect.

These structural incompatibilities between the two theories were pointed out by Hommel (2011b), who doubted that the attentional account can rely on PMTA. The existence of such incompatibilities is not overly surprising since both theories were developed to explain results obtained with different cognitive tasks: Whereas the attentional shift account aimed to explain how a stimulus is spatially coded as left or right and why maintaining the attentional focus on the centre of the screen impeded the Simon effect (Nicoletti \& Umilta, 1994), the PMTA aimed to explain the pattern of RT observed when participants responded to a target stimulus that was preceded by an invalid cue (Rizzolatti, Riggio, Dascola, \& Umiltà, 1987). This study reported larger Simon effects as the distance between an invalid cue and the subsequent target was longer. To explain these results, it was assumed that an attentional shift creates a motor program based on the direction of the spatial attention and the distance to the new attentional focus. In order to explain the Simon effect, the PMTA needs a permanent central fixation point (Rizzolatti, Riggio, Dascola, \& Umiltà, 1987; Sheliga, Craighero, Riggio, \& Rizzolatti, 1997). Otherwise, the PMTA would be unable to explain the interference produced by an incompatible trial that appears on the currently attended location. Critically, the assumption of a fixed focus on the centre of the screen directly contradicts the essential assumption of the attentional shift account (i.e., the response based on the stimulus location is activated by an attentional shift).

The referential coding account provided an alternative explanation of the formation of spatial codes that give rise to the Simon effect. Specifically, according to this view, spatial and non-spatial stimulus features are simultaneously processed. Just like with non-spatial features, such as colour or shape, spatial codes according to all available spatial reference frames are automatically created, such as regarding hemispace (i.e., the absolute location of the stimulus in the display), hemifield (i.e., the stimulus location in the fovea) (Lamberts, Tavernier, \& d'Ydewalle, 1992), and relative location concerning the alternative stimulus location (Hommel \& Lippa, 1995). These stimulus spatial codes interact with response representations and prime overlapping spatial codes of the response representations. Thus, coding a stimulus as left or right with respect to any reference frame would activate any response sharing any of these spatial codes (Hommel, Müsseler, Aschersleben, \& Prinz, 2001; Hommel, 2011a). In line with the "late-attentional" PMTA, the response based on the stimulus location and the response defined in the task instructions are activated right after their respective stimulus features have been coded (Hommel, 1993).

\section{Time-difference models}

Models assuming that multiple response representations can be concurrently primed by different stimuli or stimulus features necessarily render the timing between these stimuli or stimulus aspects an interesting factor. In the Simon task, the relevant stimulus feature, which is commonly a particular colour or shape, is competing with location, a feature that is notorious for being processed particularly fast. The irrelevant spatial feature is thus likely to be processed faster than the relevant non- 


\subsection{Relationships between attention and action}

Early vs. late attentional shift accounts

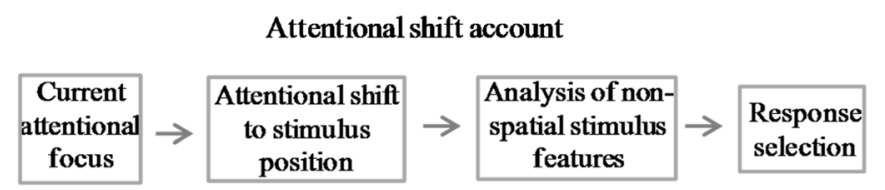

Pre-motor theory of attention

\begin{tabular}{|c|c|c|c|}
\hline $\begin{array}{c}\text { Current } \\
\text { attentional } \\
\text { focus }\end{array}$ & $\rightarrow \begin{array}{c}\text { Coding all } \\
\text { the } \\
\text { stimulus } \\
\text { features }\end{array}$ & \multicolumn{1}{c|}{$\begin{array}{c}\text { Attentional } \\
\text { shifting }\end{array}$} \\
\hline
\end{tabular}

\subsection{Sequential modulation of the Simon effect Top-down vs. button-up cognitive control mechanisms}

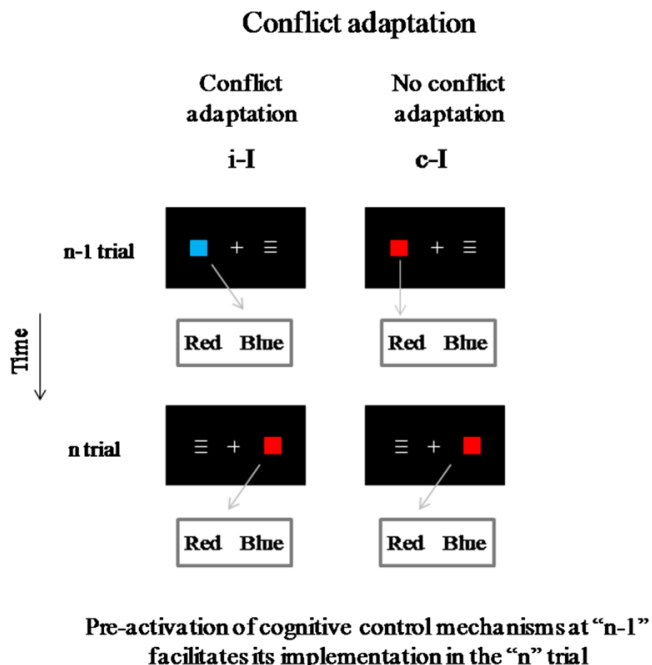

Fig. 2 Relationship between attention and action. The relationship between attention and action was explained by using earlier attentional accounts (e.g., Attentional Shift Account) and later attentional accounts

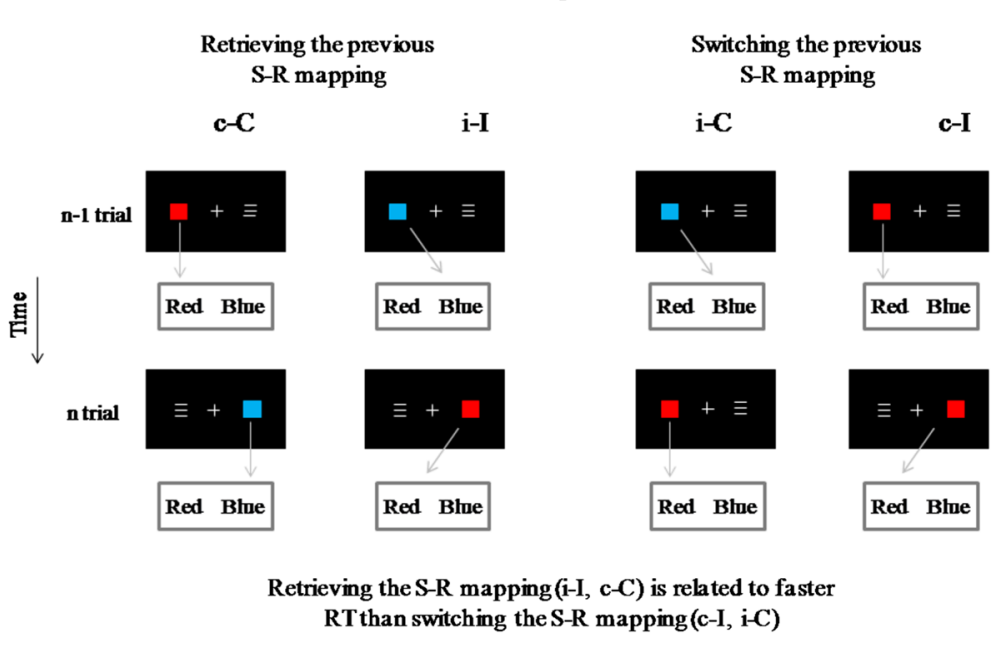

(e.g., Premotor Theory of Attention) (top panel). Sequential modulations of the Simon effect were explained according to conflict adaptation, feature integration, and a combination of both accounts (bottom panel)

location induces a fast but transient activation of the response that spatially corresponds to the stimulus. Given that translating the relevant stimulus feature into the activation of the correct response takes more time, location-induced activation may be sometimes already decayed so that no response conflict takes place and, thus, interference is not delayed. This is the more likely the sooner location activates the corresponding response and the longer the translation of the relevant stimulus feature into the activation of the correct response takes. Both predictions have been confirmed (Finkbeiner \& Heathcoat, 2016; Hommel, 1993, 1994; Ulrich, Schröter, Leuthold, \& Birngruber, 2015), suggesting that the temporal overlap between relevant and irrelevant response activation plays an important role in producing the Simon effect (see Fig. 1.3).

\section{Sequential-effect models}

Several studies reported that the Simon effect is larger after a compatible than after an incompatible trial (Stürmer, 
Leuthold, Soetens, Schröter, \& Sommer, 2002; Wühr, 2005). This phenomenon is known as the sequential effect (Gratton, Coles, \& Donchin, 1992) or Gratton effect, and it also occurs in other conflict paradigms such as Stroop and Flanker tasks (Egner \& Hirsch, 2005; Gratton, et al., 1992; Kerns et al., 2004; Lamers \& Roelofs, 2011; Mayr, Awh, \& Laurey, 2003). Two accounts have been suggested to explain these sequential effects, to which we refer in the following paragraphs.

The conflict adaptation hypothesis (Notebaert, Soetens, \& Melis, 2001; Stürmer, Leuthold, Soetens, Schröter, \& Sommer, 2002) states that experiencing a conflict increases the degree of top-down control and, as a consequence, reduces the impact of irrelevant information in a subsequent trial (Botvinick, Braver, Barch, Carter, \& Cohen, 2001). According to this view, the Simon effect is smaller after performing an incompatible trial than after performing a compatible trial because the former, but not the latter, leads to conflict (see Fig. 2.2, left panel).

The episodic retrieval hypothesis (Chen \& Melara, 2009; Duthoo, Abrahamse, Braem, Boehler, \& Notebaert, 2014; Hommel, Proctor, \& Vu, 2004; Spapè, Band, \& Hommel, 2011; Spapè \& Hommel, 2014) is based on the theory of event code (TEC) (Hommel, 2004), which represents a theoretical framework that explains the Simon effect and sequential effects through feature integration across stimulus and response (see Fig. 2.2, right panel). In short, TEC assumes that stimulus and response features are coded and integrated within the same representational system so that each trial leaves behind a binding of particular stimulus and response features. It is known that partial repetitions or alternations of stimulus and response features from trial to trial (that is, repetitions of some but not all features) impair performance presumably because the repeated features lead to the retrieval of the previous episode so that the non-repeated features compete with each other (Hommel, 2004). This implies that, in a Simon task, the sequence of two compatible or two incompatible trials - that is, compatible-Compatible (c-C) and incompatible-Incompatible (i-I) sequences - produces better performance than a compatible trial following an incompatible trial or an incompatible trial following a compatible trial - that is, incompatibleCompatible (i-C) and compatible-Incompatible (c-I) sequences (see Fig. 2.2).

Several studies obtained sequential effects even if experimental manipulations prevented associative learning or integration of S-R features (e.g. Kim \& Cho, 2014; Weissman, Jiang \& Egner, 2014). Likewise, sequential effects were obtained in studies using experimental manipulations to prevent conflict adaptation (Hommel, Proctor \& Vu, 2004; Mayr, Awh, \& Laurey, 2003; Nieuwenhuis et al., 2006). Therefore, the available results can be taken to suggest separable contributions of conflict adaptation and feature integration, or partial, but incomplete overlap of the underlying mechanisms
(Spapè, Band \& Hommel, 2011). Indeed, recent studies suggested that both mechanisms may simultaneously operate at different levels with the aim of adapting the behaviour to environmental demands (Abrahamse, Braem, Notebaert, \& Verguts, 2016; Egner, 2014). Associative learning mechanisms related to episodic memory might operate at a more concrete level, whereas attentional mechanisms related to the executive attentional system might operate at a more abstract or general level. Nevertheless, considering the difficulty to entirely prevent feature integration even in experimental designs that pursue this goal, besides the explanations that attribute the sequential effects to learning associative processes (Spapè \& Hommel, 2014; Spapè, Band, \& Hommel, 2011), some researchers argued that the conflict adaptation hypothesis could turn into an unnecessary account to explain sequential effects (Schmidt, Notebaert, \& Van Den Bussche, 2015) or that conflict adaptation may be a consequence of (rather than an alternative to) feature integration (Spapé \& Hommel, 2008; Dignath, Johannsen, Hommel \& Kiesel, 2019).

\section{Key questions}

The available models differ in various ways and they make a number of predictions that are open to neuroscientific testing. Some of these predictions imply particular brain systems that are localizable as, for instance, in the case of assumptions regarding top-down control, which would be expected to involve prefrontal cortex (PFC). However, the available models are commonly functional in nature, often without assumptions regarding the brain areas that might be involved. Accordingly, mere localization of brain activity correlated with the Simon task or the Simon effect is unlikely to suffice for the comparison and evaluation of existing models. To nevertheless provide some guidance for the discussion of the outcomes of neuroscientific studies on the Simon effect proper, as well as on sequential effects, we extracted six theoretically relevant key questions $(\mathrm{Q})$ that we use to organise our discussion. Some of them are motivated by implications or predictions that many, if not all, available models share to some degree and others are motivated by predictions that differ between models.

Q1 (early vs. late locus of the conflict or Simon effect): Some models differ with respect to the question of whether the interference from the irrelevant information takes place early or late in the processing chain. Whereas some models attributed the Simon effect to processes that are related to conflict between stimulus codes (e.g., Hasbroucq \& Guiard, 1991), most models (e.g., Zorzi \& Umiltà, 1995) attributed the effect to response conflict. Q2 (early vs. late formation of spatial codes): The attentional account of spatial coding implies a much earlier 
locus of the coding process than other coding accounts or PMTA. It will thus be interesting to see whether the available evidence points to a rather early or a rather late process associated with the Simon effect proper or the spatial coding processes that are involved.

Q3 (top-down cognitive control: support of the correct response and inhibitory control): All models imply some degree of top-down control, including the support of the representation of the correct response, which would result in interactions between areas at different levels of the processing stream, for example, between the pre-frontal cortex and both the pre-motor and motor areas (Abe \& Hanakawa, 2009). However, some but not other models assume the existence of a top-down inhibitory system in addition to that. It will thus be interesting to see whether evidence for top-down support can be found and whether evidence for top-down inhibition is also available.

Q4 (concurrent activation: feedforward and mutual inhibition): All dual-process models assume that, in incompatible trials, two response representations become active. They differ, however, with respect to the question of whether these two representations engage in direct interaction, which should involve reciprocal inhibition occurring between homologous areas (i.e., in motor cortex; see Fig. 1.4), or they are controlled by prefrontal top-down control mechanisms (feedforward inhibition). It is thus important to see whether evidence for concurrent activation and direct interactions is available.

Q5 (temporal overlap of activated responses): Some models assume that the temporal overlap between relevant and irrelevant response activation determines the size of the Simon effect. Alternatively, it may be possible that the Simon effect is due to the different magnitude of attentional allocation to relevant and irrelevant stimulus features. It would thus be interesting to see whether a relationship between temporal overlap and effect size can be confirmed by neuroscientific observations.

Q6 (sequential congruency effects: conflict adaptation and feature integration): Some models attribute the sequential modulation of the Simon effect to changes in the degree of top-down support as a function of response conflict (i.e., the conflict adaptation account). Other models attribute the sequential modulation to feature integration, which may or may not be coexisting with conflict monitoring. We thus test whether neural evidence for a role of conflict adaptation and/or feature integration is available.

To summarize, different mechanisms were proposed to explain the Simon effect proper, its dependency on spatial coding and temporal overlap, and sequential modulations of the effect. In the following sections, we review neurophysiological findings to see whether they help to evaluate the claims made by the existing models and theories and whether they shed light on the cognitive and brain mechanisms involved in the Simon effect and its various dependencies.

\section{Neurophysiological findings}

In this section, we review Simon task studies that used EEG/ ERP and TMS, whose high temporal resolution is particularly suitable for investigating the cognitive processes taking place during the Simon task performance. Also, we review fMRI studies (including findings from our performed meta-analysis, which is included as Supplementary Information), whose high spatial resolution allows obtaining detailed information about the brain regions related to the Simon effect. Techniques with high spatial (fMRI) and high temporal (EEG/ERP, TMS) resolution do not have the same utility to address the raised key questions. For instance, techniques with high temporal resolution are optimal to investigate the locus of the Simon effect or temporal relationships between attention and action whereas $\mathrm{fMRI}$ is appropriate to identify the neural sources emerging in the conflicting condition but it cannot be used to test the locus of different cognitive processes throughout the chain of processing. Therefore, for each neurophysiological technique, we focus on the key questions that can be addressed therewith.

\section{Event-related brain potentials (ERPs)}

ERP was the main technique used to investigate correlates of cognitive processes occurring during the Simon task. Here we describe the main ERP components that were used to investigate cognitive processing in the Simon task and some methodological issues that are important to design and interpret the obtained results. Afterwards, we focus on the key questions formulated at the end of the previous section.

\section{ERP components in the Simon task}

To investigate cognitive control and conflict monitoring in Simon tasks, some studies focused on frontocentral N200 and medial frontal negativity (MFN) (e.g., Masaki, Murphy, Desjardins, \& Segalowitz, 2012). Frontocentral N200 is a stimulus-locked ERP appearing 200-350ms after stimulus onset (see Fig. 3.1). It has been related to inhibition and conflict detection activity from the anterior cingulate cortex (ACC) (for a review, see Folstein \& Van Petten, 2008). MFN is a response-locked ERP that appears 50-100 ms after response execution (see Fig. 3.2). MFN was considered a later correlate of ACC activity related to conflict monitoring and response conflict detection (Bartholow et al., 2005; Van Veen \& Carter, 2002). It was labelled as error-related negativity when 


\subsection{Stimulus-locked ERPs}
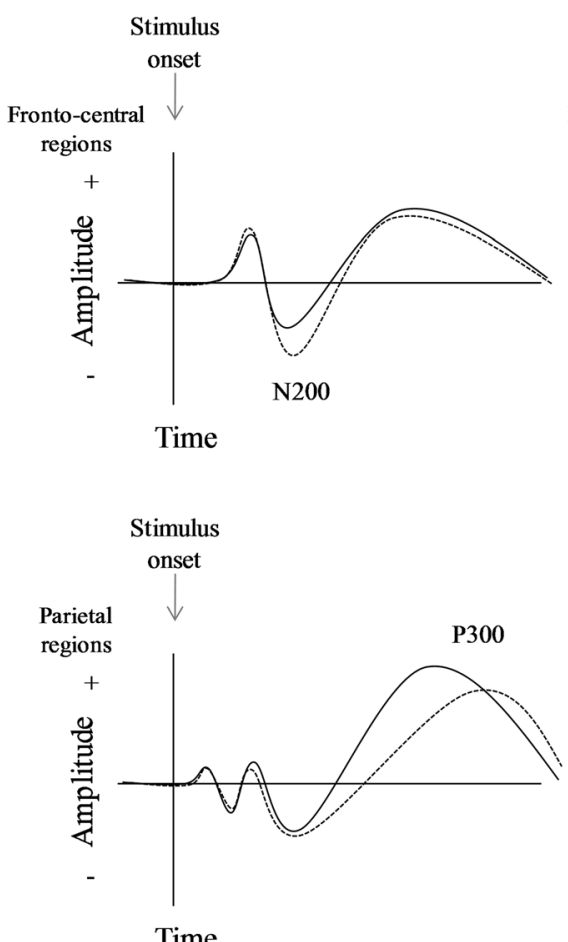

Fig. 3 Stimulus-locked ERPs (fronto-central N200, P300) and responselocked ERPs (Medial Frontal Negativity -MFN). Note that, even if we represent the waveforms for compatible and incompatible S-R trials,

following incorrect responses (for a review, see Larson, Clayson, \& Clawson, 2014).

P300 is a positive parietal ERP peaking $300-600 \mathrm{~ms}$ after stimulus presentation (see Fig. 3.1). Early studies considered the P300 as a stimulus evaluation index (Donchin \& Coles, 1988) since it correlates with stimulus discriminability (McCarthy \& Donchin, 1981; Ritter, Simson, \& Vaughan, 1972) and categorization (Kutas, McCarthy, \& Donchin, 1977). Thus, early studies assumed that $P 300$ latency could be used to distinguish between interference produced at perceptual stages (i.e., stimulus evaluation) and interference produced at post-perceptual processing stages (i.e., response selection and execution). In contrast, other studies suggested that the P300 is sensitive to the duration of the response selection stage (Ragot, 1984; Ragot \& Renault, 1981), which would be consistent with a response-selection locus of the Simon effect. Hence, P300 did not provide straightforward information to study the locus of the Simon effect (Leuthold, 2011). Interestingly, later research suggested that the parietal P300 represents an index of the time to switch the S-R binding and update working memory contents (Adrover-Roig \& Barceló, 2010), and related that process to superior parietal cortex activity (Frühholz, Godde, Finke, \& Herrmann, 2011). Indeed, in attentional switching tasks, studies reported larger parietal P300 amplitude in repeat than in switch trials

\subsection{Response-locked ERPs}

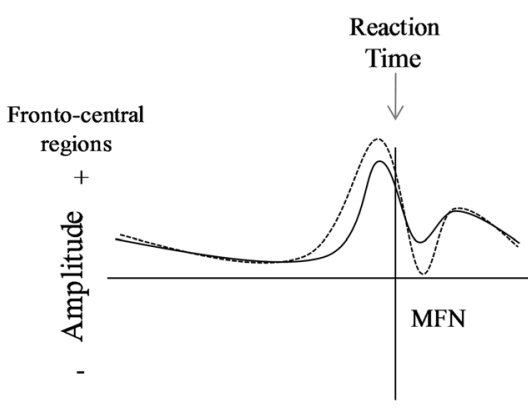

Time

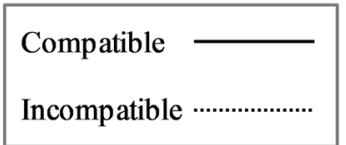

modulations of parietal P300 might be more consistently obtained by studying sequential congruency effects; that is, longer and/or lower P300 amplitude in "switch" than in "repeat" conditions

(Karayanidis, Whitson, Heathcote, \& Michie, 2011; Kieffaber \& Hetrick, 2005; Nicholson, Karayanidis, Poboka, Heathcote, $\&$ Michie, 2005). Thus, the P300 might be useful to study sequential congruency effects rather than the locus of the Simon effect.

In order to investigate the locus of the Simon effect, studies started to use the lateralised readiness potential (LRP) (De Jong, Wierda, Mulder, \& Mulder, 1988; Gratton, Coles, Sirevaag, Eriksen, \& Donchin, 1988). The LRP provides information that helps to determine whether interference effects take place at perceptual stages of processing or at response selection and execution stages. The computation of LRP is based on the readiness potential (RP) (Kornhuber \& Deecke, 1965), which is an increased negativity recorded at contralateral central sites regarding the hand involved in initiating a movement. The LRP waveform is obtained by applying a subtraction procedure to central electrodes (see Fig. 4), which removes non-motor activity and isolates the increase of activity at contralateral sites regarding the hand involved in preparing a movement (Coles, 1989; for a recent review about LRP, see Smulders \& Miller, 2012). The time from stimulus presentation to LRP onset (stimulus-locked LRP, s-LRP) provides a measure of time spent on perceptive and cognitive processes occurring before selecting a response. Also, the interval between the LRP onset and the overt response 


\subsection{Procedure to obtain the s-LRP waveform}

4.2. Procedure to obtain the LRP-r waveform

Time
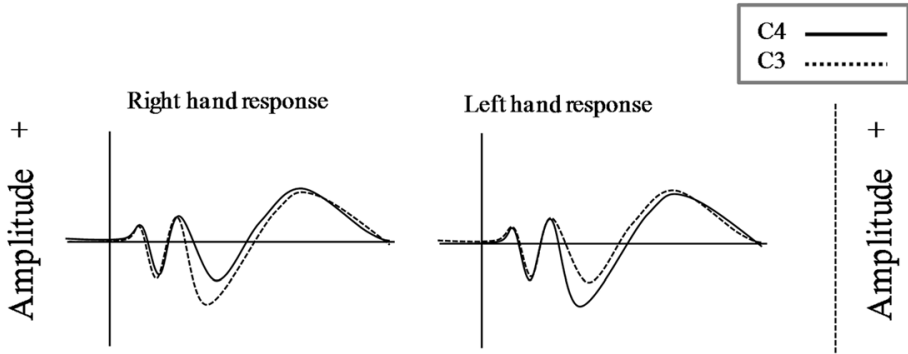

Left hand response
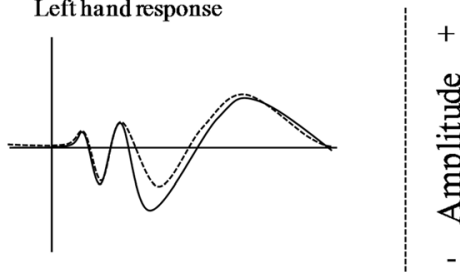

Right hand response $+$ $+$
Time
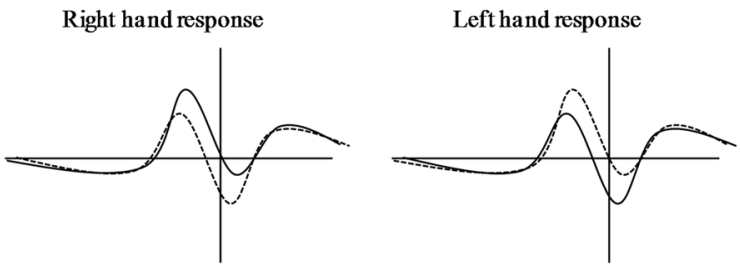

Stimulus-locked LRP waveform

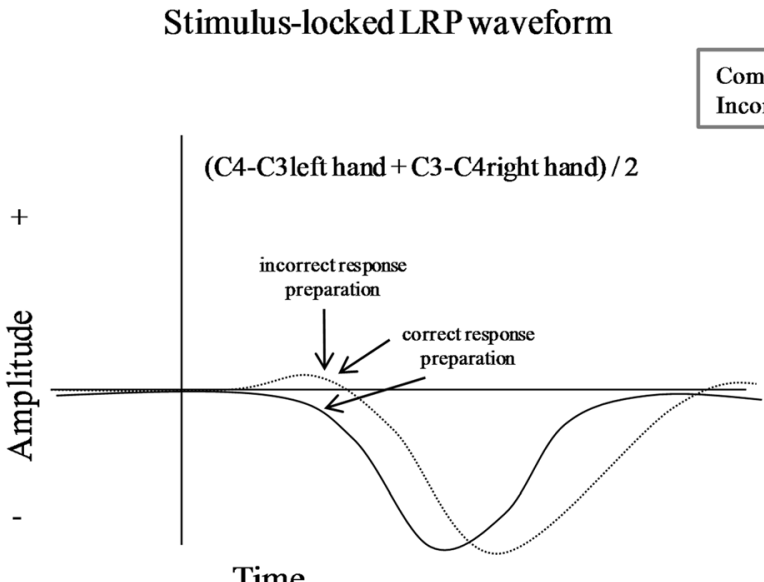

Time

Fig. 4 Stimulus-locked LRP and response-locked LRP. Representation of the double subtraction procedure used to obtain the lateralized readiness potential (LRP); stimulus-locked LRP waveforms are represented on

(response-locked LRP, LRP-r) provides an index of the duration of response execution. Therefore, as indicated in Fig. 4, the LRP provides information to distinguish between perceptual processing (time elapsed before s-LRP onset), time at which a response selection is made (s-LRP onset), and time required to execute a response or motor program (LRP-r). The clear functional interpretation of the LRP in addition to the well-defined anatomical sources (mainly within M1) and the possibility of studying the covert response activation - even if it occurs at a sub-threshold level - explains the growing use of the LRP as a tool to investigate the Simon effect and other related phenomena such as Flanker (Umebayashi \& Okita, 2010) and Stroop (Szucs, Soltész, Bryce, \& Whitebread, 2009) effects.

Even if the LRP represents a highly useful tool to study the locus of the Simon effect (Leuthold, 2011), it is blind to the contribution of each hemisphere since the LRP results from subtracting ERP waveforms recorded over both motor cortices. This limitation may be overcome by means of the Laplacian current density transformation (CSD) maps. These analyses refer to a set of mathematical computations that estimate the cortical flow of current that occurs radially through

\section{Response-locked LRP waveform}

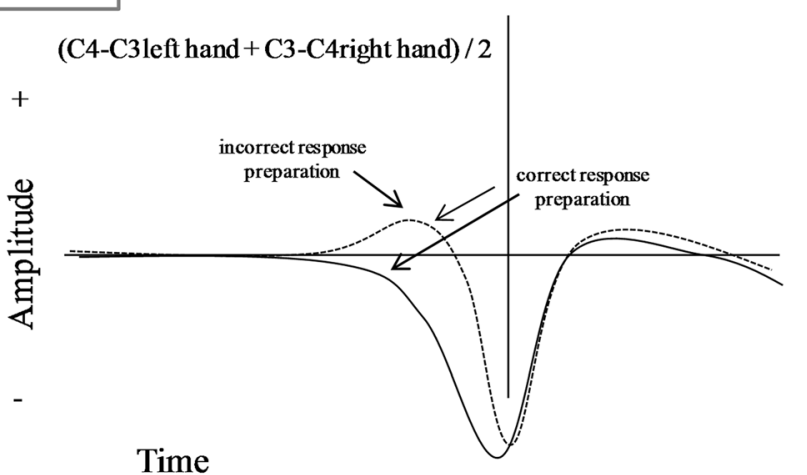

the left graphics whereas stimulus-locked LRP waveforms are represented on the right graphics

the skull and the Laplacian approach allows increasing the spatial resolution of EEG (Kayser \& Tenke, 2015). Studies have suggested that M1 activity ipsilateral to the overt response represents inhibitory activity, whereas M1 activity contralateral to the overt response is related to activity supporting response execution (Burle et al., 2015; Kayser \& Tenke, 2015). It may be useful to distinguish mechanisms to support the correct response from mechanisms to inhibit the incorrect response, which would occur in contralateral and ipsilateral hemispheres, respectively, regarding the emitted response.

Studies using the s-LRP to investigate the Simon effect evidenced a set of methodological problems that affect the interpretation of LRP results. Specifically, when a stimulus is presented in a lateralised horizontal arrangement (Sommer, Leuthold, \& Hermanutz, 1993; Valle-Inclán, 1996, Experiment 1), the eccentric display induces an asymmetrical N1 ERP component in visual regions. The LRP is affected by this asymmetry because this $\mathrm{N} 1$ waveform extends to central regions by volume conduction (Valle-Inclán, 1996, Experiment 1) - as graphically represented in Fig. 5.1 - and therefore contaminates the LRP measurement. To avoid an 


\subsection{Asymmetrical display leading to LRP/N1 overlap}

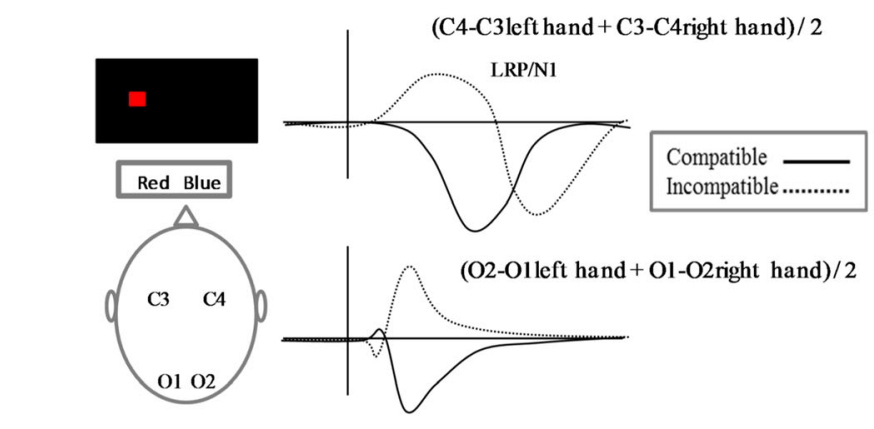

5.3. Vertical display and LRP/N2cc overlap

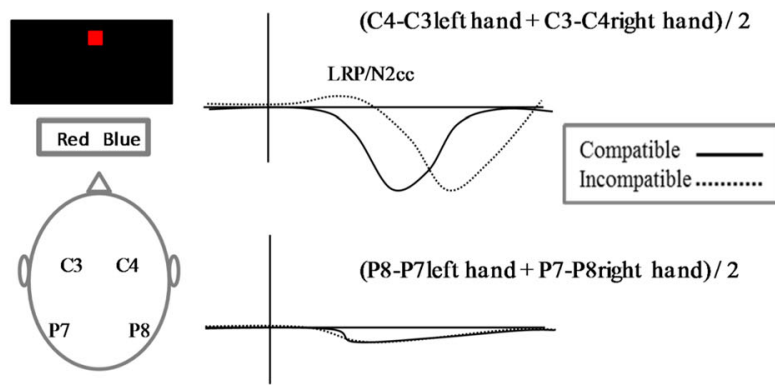

Fig. 5 Methodological procedures that were used to avoid spatial and temporal overlap of lateralised event-related potentials. N1, N2pc, N2cc, and LRP may overlap within central regions, impeding a reliable

LRP/N1 overlap, some researchers opted for the presentation of a non-target stimulus in the contralateral hemifield (Praamstra \& Oostenveld, 2003; Valle-Inclán, 1996, Experiment 2; Van der Lubbe \& Verleger, 2002). This arrangement does not alter the Simon effect (O'Leary \& Barber, 1993) and avoids the asymmetrical N1 activity at occipital regions. However, this display elicits the negativity posterior contralateral (N2pc). N2pc is an ERP waveform related to attentional selection of the target stimulus and suppression of the non-target stimulus (Eimer, 1996; Luck \& Hillyard, 1994; Woodman \& Luck, 1999), and emerges between 200 and $300 \mathrm{~ms}$ from extrastriate visual areas (Hopf et al., 2000; Luck, Girelli, McDermott \& Ford, 1997). Similar to the N1 ERP component, studies suggested that the LRP waveform is contaminated by volume conduction occurring from the N2pc sources to central electrodes (Valle-Inclán, 1996; Wascher \& Wauschkuhn, 1996), as represented in Fig. 5.2.

Interestingly, a study using a volume conduction model demonstrated that the stimulus-related lateralisation observed at central regions when studying the s-LRP was too high to be explained by passive volume conduction from neural sources related to the N2pc (Oostenveld, Praamstra, Stegeman, \& Van Oosterom, 2001). This study suggested that stimulus-related

\subsection{Bilateral display leading to LRP/N2cc/N2pc overlap}

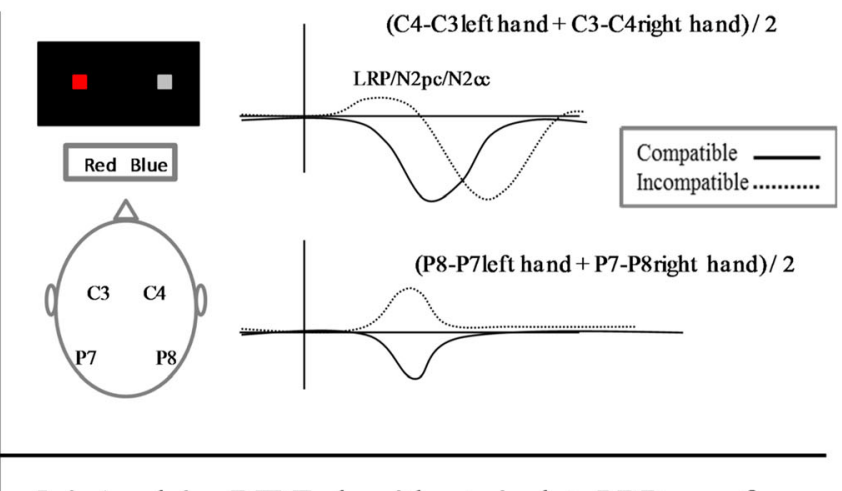

5.4. Applying RIDE algorithm to isolate LRP waveform
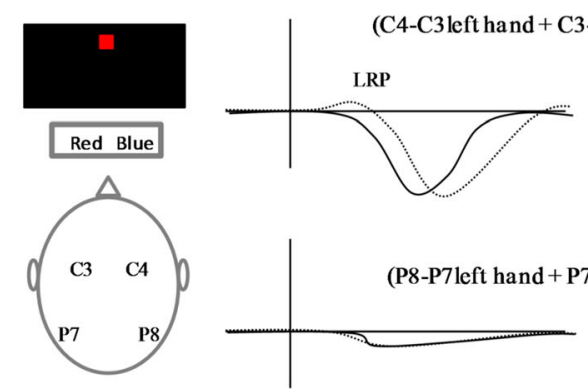

Compatible Incompatible...

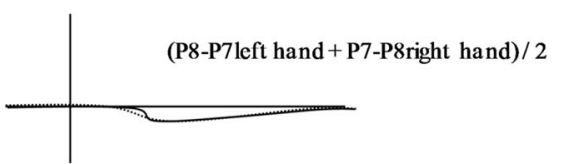

study of ERP correlates of key cognitive processes taking place during Simon task performance. This Figure shows the different problems and solutions adopted by researchers to avoid overlap of these ERPs

lateralisation observed in central regions could be associated with activity emerging at central sites (Oostenveld, Praamstra, Stegeman, \& Van Oosterom, 2001). Actually, subsequent studies related this central stimulus-related lateralization (i.e., the negativity central contralateral $(\mathrm{N} 2 \mathrm{cc})$ to dorsal premotor cortex (dPM) activity involved in preventing the response spatial tendency during Simon task performance (Praamstra \& Oostenveld, 2003; Praamstra 2006). Critically, N2cc emerges around $200 \mathrm{~ms}$ after stimulus presentation (Cespón, Galdo-Álvarez, \& Díaz, 2012; Cespón, Galdo-Álvarez, \& Díaz, 2016; Praamstra \& Oostenveld 2003; Praamstra, 2006). Thus, N2cc and LRP components overlap in similar brain regions and time windows. In order to avoid the $\mathrm{N} 2 \mathrm{cc} /$ LRP overlap, studies frequently presented the stimuli in a vertical arrangement (Stürmer, Leuthold, Soetens, Schröter, \& Sommer, 2002; Valle-Inclán, 1996, Experiment 3; Vallesi, Mapelli, Schiff, Amodio, \& Umiltà, 2005), as represented in Fig. 5.3. Apparently, it removed N2pc and N2cc components and allowed a reliable study of the LRP waveform, confirming that the Simon effect occurred during the selection of the response.

Some researchers suggested that using vertical arrangements of stimuli and responses is not the ideal solution to investigate some cognitive processes taking place during 
Simon task performance such as attention to the target stimulus and cognitive control to prevent the response spatial tendency (Leuthold, 2011). Moreover, vertical arrangements of stimuli and responses do not completely resolve the methodological problems previously discussed (Stürmer, Ouyang, Zhou, Boldt, \& Sommer, 2013).

Regarding the first issue, it is quite obvious that removing neural correlates of visuospatial attention to the lateralised target stimulus (i.e., N2pc) and cognitive control of the response spatial tendency (i.e., N2cc) during the Simon task involves renouncing to study neural mechanisms that may be crucial to understand the cognitive control of the spatial interference and the relationships between attention and action. Thus, strategies to separate LRP, N2cc and N2pc should be developed rather than designing paradigms to preclude the appearance of $\mathrm{N} 2 \mathrm{pc}$ and $\mathrm{N} 2 \mathrm{cc}$. Also, it has already been stated that LRP/N2cc overlap could not be entirely excluded in vertical arrangements (Praamstra, 2007). Actually, later studies demonstrated the presence of $\mathrm{N} 2 \mathrm{cc}$ activity in vertical arrangements (Böckler, Alpay, \& Stürmer, 2011; Stürmer, Ouyang, Zhou, Boldt, \& Sommer, 2013). In detail, Böckler, Alpay and Stürmer (2011) observed an overestimation of the Simon effect measured as the difference between the LRP onset in the incompatible and in the compatible conditions. In a later study, residual iteration decomposition algorithm (RIDE) an algorithm that is potentially capable to separate ERP components on the basis of its residual variance on every single trial (Ouyang, Herzmann, Zhou, \& Sommer, 2011) - was applied to the dataset of Böckler, Alpay and Stürmer (2011). Interestingly, after applying RIDE, the Simon effect revealed by LRP onset matched the Simon effect revealed by RT (Stürmer, Ouyang, Zhou, Boldt, \& Sommer, 2013). These findings are represented in Fig. 5.4. Therefore, appropriate experimental procedures would allow studying ERP correlates of motor activity associated with response preparation as well as the cognitive processes linked to attention to the stimulus location and inhibition of the spatial response (see Table 1).

\section{ERP components and key questions}

In this section, we examine to what extent ERP studies were useful to address the key questions formulated at the end of the previous section.

Q1: early versus late locus of the conflict (Simon effect). A large number of studies used ERPs in an attempt to identify the locus of the spatial conflict at a specific stage throughout the stream of cognitive processing; specifically, perceptual conflict (i.e., early locus of the Simon effect) versus conflict in the selection of the response (i.e., late locus of the Simon effect). These studies mainly analysed P300 and LRP components.

Pioneering studies focused on the P300. These studies reported that P300 latency was longer in incompatible conditions than in compatible conditions (as represented in Fig. 3.1) of visual (Leuthold \& Sommer, 1999; Ragot, 1984; Ragot \& Renault, 1981; Ragot \& Remond, 1979; Smulders, 1993) and auditory (Leuthold \& Schröter, 2006; Melara, Wang, Vu, \& Proctor, 2008) Simon tasks. Considering P300 as a stimulus evaluation index, these findings would be inconsistent with behavioural studies suggesting that the Simon effect occurred at the response selection stage (Acosta \& Simon, 1976; Hommel, 1995) as a result of response competition (De Jong, Liang, \& Lauber, 1994; Hommel, 1993). In fact, some researchers developed explanations of the Simon effect assuming conflict at perceptual stages to accord theoretical

Table 1. This table provides a summary of the described ERP components that were used to investigate the cognitive processing during the performance of Simon task paradigms

\begin{tabular}{|c|c|c|}
\hline ERP & Main parameters / obtaining procedure & Cognitive and neural correlates \\
\hline P300 & $\begin{array}{l}\text { Stimulus-locked ERP (s-ERP): P300 latency 300-500ms; maximum } \\
\text { amplitude at parietal sites (P3b) }\end{array}$ & $\begin{array}{l}\text { Working memory / update (switch S-R bindings); } \\
\text { Parietal and temporal areas }\end{array}$ \\
\hline LRP & $\begin{array}{l}\text { s-ERP; Subtraction procedure: } \\
\text { (C4-C3 left hand + C3-C4 right hand) / } 2 \\
\text { LRP onset: 200ms; LRPlatency: Reaction Time }\end{array}$ & $\begin{array}{l}\text { Distinction between perceptual and response preparation processes; } \\
\text { Primary motor cortex }\end{array}$ \\
\hline $\mathbf{N} 200$ & s-ERP; N2 latency: 200-350ms; Fronto-central sites & $\begin{array}{l}\text { Conflict monitoring (strength of pre-response conflict); } \\
\text { Anterior cingulate cortex }\end{array}$ \\
\hline N2pc & $\begin{array}{l}\text { s-ERP; Subtraction procedure: } \\
\text { (PO8 - PO7 left hemifield + PO7 - PO8 right hemifield / 2) } \\
\text { N2pc peak latency: } 200-300 \mathrm{~ms}\end{array}$ & $\begin{array}{l}\text { Visuospatial attention to target / suppression of non-target stimulus; } \\
\text { Parieto-occipital and temporal cortices }\end{array}$ \\
\hline N2ce & $\begin{array}{l}\text { s-ERP; Subtraction procedure: } \\
(\mathrm{C} 4-\mathrm{C} 3 \text { left hemifield }+\mathrm{C} 3-\mathrm{C} 4 \text { right hemifield / 2) } \\
\text { 200-300ms after stimulus; Pre/central sites }\end{array}$ & $\begin{array}{l}\text { Inhibition of the response spatial tendency; } \\
\text { Dorsal premotor cortex }\end{array}$ \\
\hline MFN & $\begin{array}{l}\text { Response-locked ERP (r-ERP); MFN latency: } 50-100 \mathrm{~ms} \text { after response; } \\
\text { Fronto-central sites }\end{array}$ & $\begin{array}{l}\text { Conflict monitoring (detects the need of additional cognitive control } \\
\text { after response execution); } \\
\text { Anterior cingulate cortex }\end{array}$ \\
\hline
\end{tabular}


developments to the interpretation of P300 results (Hasbroucq \& Guiard, 1991; Stoffels, van der Molen, \& Keuss, 1989). In contrast, other studies highlighted that the P300 was sensitive to the duration of the response selection stage (Ragot, 1984; Ragot \& Renault, 1981), which is consistent with a responseselection locus of the Simon effect. Subsequent studies suggested that the P300 waveform emerged from brain activity representing a link between stimulus evaluation and response selection processes (Verleger, 1997; Verleger, Jaśkowski, \& Wascher, 2005). In sum, even if most of the studies reported that P300 latency is longer in incompatible than in compatible conditions, the stage of processing related to this P300 modulation remains unclear. This circumstance limited the utility of the P300 to determine the stage of cognitive processing that reflects the Simon effect.

Early studies using the LRP located the Simon effect at the response selection stage, irrespective of whether stimuli and responses were arranged horizontally (De Jong, Liang \& Lauber, 1994; Praamstra \& Oostenveld, 2003; Sommer, Leuthold \& Hermanutz, 1993; Valle-Inclán, 1996; Van der Lubbe \& Verleger, 2002) or vertically (Stürmer, Leuthold, Soetens, Schröter, \& Sommer, 2002; Valle-Inclán, 1996). In the incompatible conditions of these studies, the s-LRP waveform shows that selection of the correct response is delayed by a transitory (and sub-threshold) preparation of the response based on the stimulus location (see Fig. 4.1, bottom part). These results provided strong support for the Simon effect as an interference effect taking place at the response selection stage.

Also, a small number of studies investigated the locus of the Simon effect by using the LRP-r (see Fig. 4.2). Studies revealed earlier LRP-r onset in the compatible condition than in the incompatible condition (Cespón, Galdo-Álvarez \& Díaz, 2013a; Cespón, Galdo-Álvarez, \& Díaz, 2013b; Shang, Fu, Qiu, \& Ma, 2016). These results suggested that the execution of the motor program is faster in the incompatible than in the compatible condition. These findings were also reported by studies using other SRC paradigms, such as the Flanker task (WildWall, Falkenstein, \& Hohnsbein, 2008), and might represent compensatory mechanisms to speed up the correct response after a transitory and sub-threshold selection of the incorrect response.

Q2: early versus late formation of spatial codes. The high temporal resolution of ERP may be useful to test theories proposing different temporal relationships between attention and action. Specifically, whereas the attentional shift account proposes that a stimulus location is first attended and the response selection is made in an ulterior stage in the chain of cognitive processing (i.e., early attentional accounts), the PMTA and the coding referential account suggest that spatial encoding and reaction to the attended location are cognitive processes occurring at the same time (i.e., late attentional accounts).

The existence of relationships between an attentional shift - as revealed by N2pc - and the Simon effect (Van der Lubbe, Abrahamse, \& De Kleine, 2012; Van der Lubbe, Jaskowśki, \& Veleger, 2005) was taken as electrophysiological evidence for the PMTA (Van der Lubbe, Abrahamse, \& De Kleine, 2012), which would be consistent with the late formation of spatial codes. However, the PMTA as well as the attentional shift account predict an association between attentional shifts and Simon effect. Studying the temporal relationship between formation of spatial codes and motor preparation is crucial to clarify whether those ERP findings are in line with PMTA. Furthermore, it should be taken into account that attentional selection indicated by N2pc may actually occur previously to the formation of the spatial code that triggers the spatially compatible response, as suggested by the obtaining of the Simon effect in bilateral arrays (O'Leary \& Barber, 1993). Nevertheless, overlap between correlates of attentional selection (N2pc) and motor preparation (e.g., LRP) within the same time window would suggest a late formation of the spatial code. To date, we are not aware of studies using these ERP components to address this question. Even so, studies that used N2pc and LRP to investigate other issues show that both ERP components take place within the same time window (e.g., Cespón, Galdo-Álvarez, \& Díaz, 2013b; Praamstra \& Oostenveld, 2003; Van der Lubbe \& Verleger, 2002), which would be consistent with late attentional accounts (e.g., PMTA, referential coding account).

There is also evidence of dissociations between attentional shifting and the Simon effect (Valle-Inclán, Hackley, \& De Labra, 2003; Valle-Inclán, Sohn, \& Redondo, 2008; ValleInclán \& Redondo, 1998). Specifically, several studies (Valle-Inclán, Hackley, \& De Labra, 2003; Valle-Inclán, Sohn, \& Redondo, 2008) used Simon-type tasks in which the target stimulus was presented to the left or right eye only. RTs and LRPs related to the Simon effect between stimulated eye and response location were obtained under conditions where participants consciously perceived the stimulus as appearing in the center of the screen and were not aware of which eye was stimulated. Surprisingly, reliable Simon effects were obtained even under these conditions, that is, even in the absence of spatial cues that would allow (re-)orienting attention. In addition, Valle-Inclán and Redondo (1998) reported the absence of any stimulus-induced activation of the incorrect response (as revealed by LRP) before the information about the mapping of the non-spatial (but task-relevant) stimulus feature to the response button was known; this implies that knowledge about associations between the relevant feature of the stimulus and the hand of response (i.e., definition of the $\mathrm{S}$ $\mathrm{R}$ associations) is crucial for activating the direct visuomotor pathway - in contradiction to the widespread characterization 
of this pathway as "automatic". Altogether, these studies suggest that the information about S-R associations plays a crucial role in the occurrence of the Simon effect (Valle-Inclán \& Redondo, 1998) and that spatial codes may be formed in the absence of attentional shifts (Valle-Inclán, Hackley, \& De Labra, 2003; Valle-Inclán, Sohn, \& Redondo, 2008), which would be in line with the referential coding account (Hommel, 2011a).

\section{Q3: Top-down cognitive control: Support of the correct response versus inhibitory control mechanisms. Some theoretical models proposed that correct performance in a Simon task relies on a top-down mechanism to select the correct response, whereas other models proposed ad- ditional mechanisms to inhibit the incorrect response. The existence of both mechanisms (i.e., support of the correct response and inhibition of the incorrect response) would be less parsimonious than assuming a top-down control responsible for selecting the correct response. So, we review to what extent ERP studies provided evidence for both mechanisms.}

Studies using Laplacian transformed ERP analyses to investigate brain activity within both M1 separately suggested the existence of a top-down control mechanism responsible to support the correct response (i.e., a negativity in contralateral M1) and another one to inhibit the incorrect response (i.e., a positivity in ipsilateral M1) (Burle, van den Wildenberg, Spieser, \& Ridderinkhof 2016; Meckler et al., 2010; Servant, White, Montagnini \& Burle, 2016). These studies usually reported that neural activity in ipsilateral M1 - but not in contralateral M1 - correlated with the degree of required inhibition. For instance, Burle, van den Wildenberg, Spieser and Ridderinkhof (2016) used a Go/No-Go Simon task in which the stimulus frequency of compatible and incompatible trials was manipulated. The less frequent incompatible trials were, the higher the conflict was. Moreover, inhibitory activity - as revealed by neural activity recorded over the ipsilateral M1 regarding the required response hand - was higher when incompatible trials were less probable to occur; however, activity associated with response execution (i.e., neural activity contralateral to the overt response) did not change according to the experimental conditions.

Some studies showed that enhanced amplitude of N2cc was associated with greater Simon conflict (Cespón, GaldoÁlvarez, \& Díaz, 2012; Cespón, Galdo-Álvarez, \& Díaz, 2016; Leuthold \& Schröter, 2006). Moreover, several studies showed that the amplitude of frontocentral N200 was larger in incompatible than compatible trials (Cao, Cao, Yue, \& Wang, 2017; Carriero, Zalla, Budai, \& Battaglini, 2007; Chen \& Melara, 2009; Hoppe, Küper \& Wascher, 2017; Li et al., 2015; Melara, Wang, Vu, \& Proctor, 2008; Wang, Li, Zheng, Wang, \& Liu, 2014). Given that the degree of neural activity to support the correct response does not change according to S-R compatibility (Burle, van den Wildenberg, Spieser, \& Ridderinkhof, 2016), larger frontocentral N200 and N2cc in incompatible than compatible trials were taken to point to the existence of additional neural activity deployed in incompatible conditions to inhibit the response based on the stimulus location.

Q4: concurrent activation: Feedforward and mutual inhibition. LRP studies showing a competition between alternative responses at the response selection stage (De Jong, Liang, \& Lauber, 1994; Praamstra \& Oostenveld, 2003; Sommer, Leuthold \& Hermanutz, 1993; Stürmer, Leuthold, Soetens, Schröter, \& Sommer, 2002; ValleInclán, 1996; Van der Lubbe \& Verleger, 2002) clearly supported the main assumption of dual-route models, which considers that the Simon effect is the result of a competition between the response based on stimulus location and the response based on task instructions (De Jong, Liang, \& Lauber 1994; Hommel, 1993; Tagliabue, Zorzi, Umiltà, \& Bassignani, 2000). Some studies investigated whether response competition is related to either top-down inhibition or reciprocal inhibition between response representations in the two motor cortices, a relevant question considering that reciprocal inhibition between competing responses is a mechanism assumed by some dual-route models (e.g., Zorzi \& Umiltà, 1995) but not by others (e.g., Kornblum, Hasbroucq, \& Osman, 1990).

As already mentioned, recent research reported that motor activity associated with inhibition of the spatial response was higher when the incompatible trials were less frequent, while activity related to response activation did not depend on frequency (Burle, van den Wildenberg, Spieser, \& Ridderinkhof, 2016). Thus, activation and inhibition of homologue areas did not correlate. These results were in line with some previous studies (Meckler et al., 2010; Vidal, Burle, Grapperon, \& Hasbroucq, 2011) and favoured the idea of top-down inhibition - i.e., inhibition processes occurring throughout a pathway of processing (Servant, White, Montagnini, \& Burle, 2016) - over that of reciprocal inhibition - i.e., inhibition of a response through its competing response (Bogacz, Brown, Moehlis, Homes, \& Cohen, 2006; Usher \& McClelland, 2001).

Q5: temporal overlap of the activated responses. Some studies stated that the temporal overlap between the relevant and the irrelevant response activation determines the size of the Simon effect. Given the high temporal resolution of the ERP technique, it is a suitable tool to study the time required for processing relevant and irrelevant stimulus features and to investigate whether the size of the 
Simon effect depends on the temporal relationship between the processing of relevant and irrelevant stimulus features. A couple of ERP studies investigated this issue and provided evidence that temporal overlap of the activated responses might indeed determine the size of the Simon effect.

Wascher (2005) reported that the size of the interference was reduced when the time between the stimulus localisation (as revealed by $\mathrm{N} 2 \mathrm{pc}$ latency) and the manual response was increased. This study itself does not entirely clarify whether such decrease in the interference size might be automatic or a result of deployment of inhibitory mechanisms. Another study carried out by Strack, Kaufmann, Kehrer, Brandt and Stürmer (2013) shed some light on this issue. Specifically, these authors showed reduced Simon effect and smaller frontocentral N200 amplitude when advancing information about the stimulus location of the next trial. This result suggests less need to deploy cognitive control activity when the processing of the stimulus location and the processing of the relevant dimension are separated in time. Therefore, the degree of temporal overlap between the processing of the relevant and the irrelevant stimulus dimensions influences the magnitude of the Simon effect.

Q6: Sequential congruency effects: Conflict adaptation and feature integration. Studies investigating ERP modulations by sequential effects focused on N200, P300 and LRP components. Sometimes these studies used experimental manipulations that prevented the integration of S$\mathrm{R}$ features or the conflict adaptation to shed light on the cognitive mechanism underlying sequential congruency effects.

As previously stated, several studies focused on modulations of the frontocentral N200, like the study by Cao, Cao, Yue and Wang (2017). These authors reported larger frontocentral N200 in incompatible than compatible trials. However, the effect was reversed when presenting $25 \%$ of compatible and $75 \%$ of incompatible trials; that is, under these experimental conditions, frontocentral N200 was larger in compatible trials than in incompatible ones. This result indicates that a higher cognitive control was required to implement the appropriate response in the compatible condition than in the incompatible one, which argues for contextdependent learning of S-R associations and against conflict adaptation since, from a conflict adaptation standpoint, high rate of trials may reduce the conflict to zero but never reverse the effect. Another study carried out by Li et al. (2015) reported larger frontocentral N200 in i-C than c-C trials (i.e., i-C trials required more cognitive control than $\mathrm{c}-\mathrm{C}$ trials), but differences between i-I and c-I trials did not reach statistical significance. Once again, these results would not be in line with conflict adaptation, which should distinguish between i-I and c-I sequences but not necessarily between $\mathrm{c}-\mathrm{C}$ and i-C sequences, where the subject responds to a non-conflicting stimulus.

Some studies used experimental manipulations to dissociate conflict adaptation from feature integration. A study carried out by Chen and Melara (2009) reported similar sequential congruency effects on behavioural and ERP (N200, P300) data for sequences involving incompatible and neutral trials. As conflict adaptation cannot occur in incompatible trials preceded by neutral trials, these results suggested feature integration as the main factor contributing to sequential effects (Chen \& Melara, 2009). Another recent study combined stimuli arranged vertically and horizontally within the same Simon task (Hoppe, Küper, \& Wascher, 2017). Sequential effects were found only when the spatial dimension was repeated, which would be in line with the feature integration account. In addition, sequential effects were accompanied by modulations of the P300 - which reflect neural activity to switch the S-R binding and update working memory contents (AdroverRoig \& Barceló, 2010; Kieffaber \& Hetrick, 2005) - but not by modulations of the frontocentral N200 - an ERP component closely related to cognitive control (Folstein \& Van Petten, 2008; Van Veen \& Carter, 2002). The results of Hoppe, Küper and Wascher (2017) were consistent with previous studies in which sequential effects were eliminated by using experimental manipulations that impeded a perceptual integration of S-R features (Spapè, Band, \& Hommel, 2011; Spapè \& Hommel, 2014).

Studies also used LRP as a tool to investigate sequential modulations of the Simon effect. Overall, these studies typically showed diminished incorrect response preparation - revealed by a positive dip of the LRP (see Fig. 3) - in incompatible trials that were preceded by another incompatible trial, as compared to incompatible trials that were preceded by a compatible trial (Stürmer \& Leuthold, 2003; Stürmer, Leuthold, Soetens, Schröter \& Sommer, 2002). In a more recent study, Spapè, Band and Hommel (2011) showed that these sequential effects on the LRP were eliminated by using experimental manipulations to avoid the integration of S-R features, thus supporting the account based on feature integration. Other studies suggested that it might not be the conflict itself that is driving sequential effects, as implied by the conflict-monitoring approach, but the unpleasant affective consequences of the conflict (Dreisbach \& Fischer, 2015). In line with this view, Fröber, Stürmer, Frömer and Dreisbach (2017) conducted a study with a vertical Simon task, in which participants were asked to judge a trial as pleasant or unpleasant on a Likert scale. Unpleasant judgements correlated with the strength of conflict in the next trial, as revealed by behavioural and LRP results.

In summary, most ERP research is consistent with feature integration rather than conflict adaptation as a mechanism 
underlying sequential congruency effects. Future studies might analyse other ERP components related to conflict monitoring and cognitive control such as MFN or N2cc, which may provide additional information about modulations of neural activity underlying sequential congruency effects.

\section{EEG spectral power}

Some EEG studies analysed the spectral power obtained in different experimental conditions of the Simon task. These studies mainly focused on the theta band, which was related to cognitive control. We briefly describe this frequency band and EEG studies shedding light on the third and sixth key questions.

\section{EEG spectral power and the Simon task}

A variety of studies yielded Simon conflict effects in different frequency bands, in particular the theta band that comprises 4$8 \mathrm{~Hz}$. The theta band is modulated by a wide range of attentional and memory tasks (Jacobs, Hwang, Curran, \& Kahana, 2006; Missonnier et al., 2006) but also feedback and error processing (Andreou et al., 2017; Bernat, Nelson, \& BaskinSommers, 2015; van Driel, Ridderinkhof, \& Cohen, 2012). It is negatively correlated with the default mode network and usually increases with higher task demands (Gundel \& Wilson, 1992; Scheeringa et al., 2008). A reliable finding is the increase of theta-band activity over mid-frontal electrodes in incompatible compared to compatible trials of the Simon task between 300 and $600 \mathrm{~ms}$ after stimulus onset (Cao, Cao, Yue, \& Wang, 2017; Cohen \& Donner, 2013; Cohen \& Ridderinkhof, 2013; Gulbinaite, van Rijn, \& Cohen, 2014; Li et al., 2015; Töllner et al., 2017; van Driel, Swart, Egner, Ridderinkhof, \& Cohen, 2015; Vissers, Ridderinkhof, Cohen, \& Slagter, 2018; Wang, Li, Zheng, Wang, \& Liu, 2014). These studies suggested that increased midfrontal theta activity represents a neural marker of cognitive control. In this context, it was proposed that midfrontal theta oscillations provide a reference frame for monitoring and orchestrate a broad control network of frontal, parietal, and subcortical brain areas (Cavanagh \& Frank, 2014; Cohen, 2014). Neural generators of midfrontal theta effects were localised in the ACC and the SMA/pre-SMA (Cohen \& Ridderinkhof, 2013; Töllner et al., 2017).

\section{EEG spectral power studies and key questions}

Q3: Top-down cognitive control: Support of the correct response versus inhibitory control mechanisms. As previously stated, theta power is related to cognitive control. A substantial number of studies reported larger midfrontal theta power in incompatible than compatible conditions during the Simon task performance (Cao, Cao, Yue, \& Wang, 2017; Cohen \& Donner, 2013; Gulbinaite, van Rijn, \& Cohen, 2014; Li et al., 2015; Töllner et al., 2017; van Driel, et al., 2015; Vissers, Ridderinkhof, Cohen, \& Slagter, 2018; Wang, Li, Zheng, Wang, \& Liu, 2014). Furthermore, several studies found increased connectivity between prefrontal and parietal areas in incompatible compared to compatible trials within the theta band (Cohen \& Ridderinkhof, 2013; Gulbinaite, van Rijn, \& Cohen, 2014). Overall, in line with ERP research, these studies attributed increased theta power to enhanced cognitive control and reported the existence of a frontoparietal network involved in executive control during the performance of the Simon task.

The previously reported midfrontal theta power effects are evident in a variety of paradigms such as Simon, Flankers, Go/ No-go (Nigbur, Ivanova, \& Stürmer, 2011), response priming (Pastötter, Dreisbach, \& Bäuml, 2013) or Stroop (Hanslmayr et al., 2008) tasks, which might point to a universal control system. Even so, theta band-related conflict effects slightly differ between different types of conflict tasks with respect to temporal dynamics and neural sources (Cohen \& Cavanagh, 2011; Nigbur, Ivanova, \& Stürmer, 2011; Wang, Li, Zheng, Wang, \& Liu, 2014). For instance, increased theta power in left ACC and left pre-SMA was reported in priming tasks (Pastötter, Dreisbach, \& Bäuml, 2013), whereas bilateral increase of theta was showed in Simon (Töllner et al., 2017) or Stroop (Hanslmayr et al., 2008) tasks in dorsal ACC. Thus, as concluded by Töllner et al. (2017), increase of medial frontal theta power in different conflicting tasks is probably reflecting diverse phenomena.

Q6: Sequential congruency effects: Conflict adaptation and feature integration. As previously highlighted, enhancement of midfrontal theta power is usually observed in incompatible compared to compatible trials (e.g., Cohen \& Ridderinkhof, 2013; Töllner et al., 2017). This conflict-induced effect is diminished when the current trial is preceded by an incompatible trial, which was interpreted according to conflict adaptation (Cohen \& Ridderinkhof, 2013). However, most of studies about theta power modulations are not in line with the conflict adaptation theory (Cao, Cao, Yue, \& Wang, 2017; Gulbinaite, van Rijn, \& Cohen, 2014; Van Driel, Swart, Egner, Ridderinkhof, \& Cohen, 2015). Van Driel, Swart, Egner, Ridderinkhof and Cohen (2015) used temporal cues to indicate trials with high versus low conflict probability. Cues that predict a high probability of conflict should be associated with the upregulation of top-down control and attenuate the impact of conflicting information. However, conflict-related increase of midfrontal theta was only evident in trials with "high-probability-cues". Results from Cao, Cao, Yue and Wang (2017) showed a reversed Simon effect by decreasing the proportion of 
compatible trials, which cannot be explained by conflict adaptation. Authors interpreted these results as evidence for contingency learning account formulated to explain the reversed Stroop effect when increasing the ratio of incongruent trials (Atalay \& Misirlisoy, 2012; Grandjean et al., 2013). As argued by those authors, conflict adaptation might result in a zero interference effect; that is, RT would be the same for compatible and incompatible trials. Nevertheless, it is difficult to understand how conflict adaptation may lead to a reversed interference effect (Cao, Cao, Yue, \& Wang, 2017).

Some studies reported greater midfrontal theta power in i-C than c-C and in c-I than i-I trials (e.g., Gulbinaite, van Rijn, \& Cohen, 2014; Töllner et al., 2017). The difference observed between $\mathrm{c}-\mathrm{C}$ and $\mathrm{i}-\mathrm{C}$ trials would be more parsimoniously explained by feature integration than conflict adaptation. For differences between i-C and c-C trials, it has been argued that participants may adopt a more conservative criterion after experiencing a conflict and the RT is slowed down (Botvinick, Braver, Barch, Carter, \& Cohen, 2001; Li et al., 2015). Although it is a plausible explanation, this interpretation involves assuming two different mechanisms to explain sequential effects (i.e., conflict adaptation in i-I trials and changing to a more conservative criterion in $\mathrm{i}-\mathrm{C}$ trials), which results in a less parsimonious model than feature integration.

\section{Transcranial magnetic stimulation (TMS)}

TMS is a non-invasive brain stimulation technique that consists of placing a coil device over the scalp to apply magnetic fields that interact with neural activity and evoke action potentials (Barker et al., 1985). The TMS is able to transiently disrupt the normal functioning of the stimulated area by adding "neural noise" (Miniussi, Harris, \& Ruzzoli, 2013) (i.e., it may be metaphorically considered as an electrically short-time "lesion"), which allows inferring the causal role of the stimulated area during the performance of a given cognitive task.

To obtain information about the role of a given area during the performance of a cognitive or motor task, TMS may be applied in different modalities. Specifically, TMS may be applied both online (i.e., during the performance of a task) and offline (i.e., before performing the task) (for a review about TMS studies in SRC tasks, see Olk, Peschke, \& Hilgetag, 2015). Overall, online approaches involve delivering a single TMS pulse (spTMS) or train of pulses (it may be considered a sort of repetitive TMS -rTMS) to a given area at a specific time after the appearance of the stimulus. Also, it may consist of a paired-pulse (ppTMS) onto the same or different areas, which allows studying several aspects of the brain connectivity (Koch \& Rothwell, 2009). On the other hand, offline approaches involve applying TMS pulses in a repetitive manner (rTMS) for several minutes, which induces long-lasting changes in the stimulated area before performing the task (for a review about TMS, see Burke, Fried, \& Pascual-Leone, 2019).

\section{TMS studies in the Simon task}

Studies using the Simon task have applied TMS over parietal, frontal, and motor areas to investigate how stimulation modulated the Simon effect (i.e., RTs in the compatible and incompatible conditions) and/or motor evoked potentials (MEPs) recorded from peripheral muscles. These areas represent neural regions supporting Simon task processing, as they have an important role in visuospatial attention, cognitive control, and response preparation processes, respectively. TMS studies using the Simon task, which are reviewed in the following subsection, provided information mainly about the second, third, fourth and sixth key questions.

\section{TMS and key questions}

Q2: Early versus late formation of spatial codes. Several studies used TMS to modify the activity of the frontoparietal network and the visuomotor processing during the performance of a Simon task with the aim of studying where and when spatial properties of the stimulus are encoded (Bardi, Kanai, Mapelli, \& Walsh, 2012; Schiff, Bardi, Basso, \& Mapelli, 2011). These studies provide evidence about the temporal window and the brain areas involved in encoding spatial attributes.

Bardi, Kanai, Mapelli and Walsh (2012) studied the activation of the spatial response by investigating the potential role of frontal eye fields (FEFs) to encode spatial attributes. They found that double-pulse TMS $(25 \mathrm{~Hz})$ at $0-40 \mathrm{~ms}$ and at 40-80 ms over left FEF after the appearance of the stimulus reduced the Simon effect for stimuli placed in the right hemifield, whereas TMS at 80-120 ms over right FEF reduced Simon effect for stimuli placed in the left hemifield. Thus, in line with the role attributed to the FEF for the control of visuospatial attention (Grosbras \& Paus, 2002), the authors concluded that the FEF plays a crucial role in encoding spatial attributes that are important for response priming (Bardi, Kanai, Mapelli, \& Walsh, 2012). As concluded by these authors, the reported results are consistent with PMTA, as the Simon effect vanishes when TMS disrupts activity in a neural region (i.e., FEF) that is crucial to program and implement saccadic movements.

In another study, Schiff, Bardi, Basso and Mapelli (2011) applied spTMS at several time intervals after the onset of the stimulus $(70,100,130,160,190,220,250 \mathrm{~ms})$ to the left and right angular gyrus $(\mathrm{AG})$ and supramarginal gyrus (SMG) 
because of their key role in orienting spatial attention and transforming sensory information into motor commands, respectively (Rushworth, Krams, \& Passingham, 2001). Evidence from Schiff, Bardi, Basso and Mapelli (2011) supported the role of right $\mathrm{AG}$ in orienting attention, as TMS over right $A G$ at $130 \mathrm{~ms}$ and over the left $A G$ at $160 \mathrm{~ms}$ after the onset of the stimulus suppressed the Simon effect. Likewise, TMS delivered over the left SMG (but not over the right hemisphere) at $160 \mathrm{~ms}$ after the onset of the stimulus eliminated the Simon effect. Thus, the authors concluded that PPC is involved in sending the irrelevant spatial information to the motor system and transforming spatial information into a code for action. In this regard, Stürmer, Redlich, Irlbacher and Brandt (2007) applied rTMS at 0-200 ms after stimulus onset over right PPC. It reduced the Simon effect of left-hand responses, which allowed the conclusion that right PPC relates to early visuomotor transformations.

Overall, evidence from TMS studies suggests that FEF plays an early role and PPC a late role in encoding the spatial information and sending a motor command (to respond on the basis of the stimulus location) to the motor system (Olk, Peschke, \& Hilgetag, 2015). In other words, FEF and PPC represent neural correlates of the direct visuomotor pathway postulated by dual-route models (e.g., De Jong, Liang, \& Lauber, 1994). Also, TMS disrupts the formation of the spatial code at an early time after stimulus presentation in areas that are important to program saccadic movements and shift attention. Even if these studies do not allow drawing strong conclusions about the temporal relationship between attention and action, they suggest that both processes may overlap within the same time window and neural structures, which would align with late attentional accounts (i.e., PMTA, referential coding account).

Q3: Top-down cognitive control: support of the correct response versus inhibitory control mechanisms. There are a set of TMS studies that may be related to the third theoretical question, as these studies shed light on which brain regions are involved in cognitive control over stimulus-induced response tendencies. Several studies targeted the dPM (Bardi, Schiff, Basso, \& Mapelli, 2015; Praamstra, Kleine, \& Schnitzler, 1999), as evidence from ERP (Praamstra, 2006; Praamstra \& Oostenveld, 2003) and intracranial recordings (for a review, see Abe \& Hanakawa, 2009) suggested that this area plays a crucial role in cognitive control to inhibit the response based on the stimulus position (Praamstra \& Oostenveld, 2003; Praamstra, 2006). Other studies stimulated the dorsolateral prefrontal cortex (DLPFC) (Stürmer, Redlich, Irlbacher, \& Brandt, 2007) and the inferior frontal gyrus (IFG) (van Campen, Kunert, van den Wildenberg, \& Ridderinkhof, 2018), which represent brain areas typically related to executive control.
Bardi, Schiff, Basso and Mapelli, (2015) applied spTMS over left and right dPM and observed that spTMS over the left dPM at 160 ms reduced the Simon effect by increasing RTs in the compatible condition. At $220-250 \mathrm{~ms}$, spTMS over the left dPM increased the Simon effect by increasing RTs in the incompatible condition. These results led to the conclusion that dPM is involved in the activation of the spatially compatible response and in response selection after the spatial conflict. Furthermore, other studies showed that spTMS over dPM slows RTs in response-choice tasks (Johansen-Berg et al., 2002; O'Shea, Sebastian, Boorman, Johansen-Berg, \& Rushworth, 2007; Schlutter, Rushworth, Passingham, \& Mills, 1998; see also Praamstra, Kleine, \& Schnitzler (1999), who reported an increased Simon effect after repetitive TMS over the left dPM between 300 and $150 \mathrm{~ms}$ before stimulus presentation).

Stürmer, Redlich, Irlbacher and Brandt (2007) applied repetitive TMS (rTMS) over left DLPFC between 500 and $300 \mathrm{~ms}$ before stimulus presentation but the Simon effect remained unchanged even if it modulated sequential effects, suggesting that DLPFC plays a role in context-dependent response control. In contrast to Praamstra, Kleine and Schnitzler (1999), Stürmer, Redlich, Irlbacher and Brandt (2007) did not find behavioural modulations when delivering rTMS over dPM. Also, van Campen, Kunert, van den Wildenberg and Ridderinkhof (2018) showed increased Simon effect after delivering rTMS over the right IFG but the effect was only evidenced at the slowest RTs (with no effects of rTMS over pre-SMA, which was attributed to a larger distance between the coil and the stimulated area). These authors concluded that the right IFG is a region involved in suppressing the response spatial tendency.

A very limited number of studies combined TMS and neuroimaging techniques to study changed activity in brain circuits involved in performing a cognitive task. An example is the study conducted by Herz et al. (2014), who combined rTMS and fMRI in an experiment in which $1 \mathrm{~Hz}$ rTMS was delivered over $30 \mathrm{~min}$ to the pre-SMA before performing a Simon task. rTMS over pre-SMA improved cognitive control over spatial responses (but only when trials contained financial incentives), which was related to increased connectivity within inferior frontal gyrus (IFG)-subthalamic nucleus pathway.

In short, TMS studies provided evidence for the existence of several areas acting in concert to deploy cognitive control mechanisms during the performance of a Simon task. That is, whereas premotor regions (i.e., dPM) seem specifically devoted to prevent the execution of incorrect stimulus-induced responses, the DLPFC seems to be involved in contextdependent response control. Figure 6 recaps TMS parameters that modulated the Simon effect by stimulating areas related to activation of the direct visuomotor pathway and allocation of cognitive control activity to prevent the response spatial 


\section{Spatio-temporal TMS parameters that modulated direct visuo- motor pathway and cognitive control}
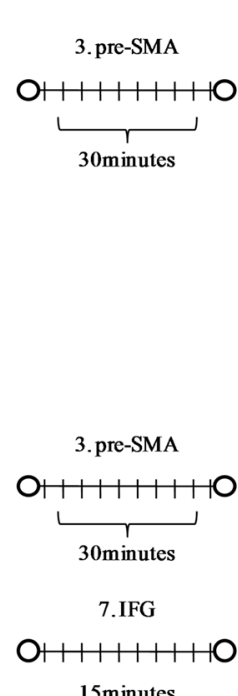

15minutes

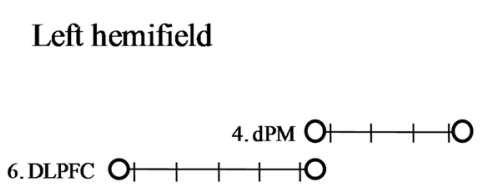

6. DLPFC O+,++10
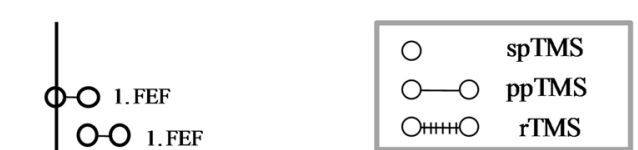

O 2.dPM

O 2.dPM

O 2.dPM

O 5.AG

O 5.SMG

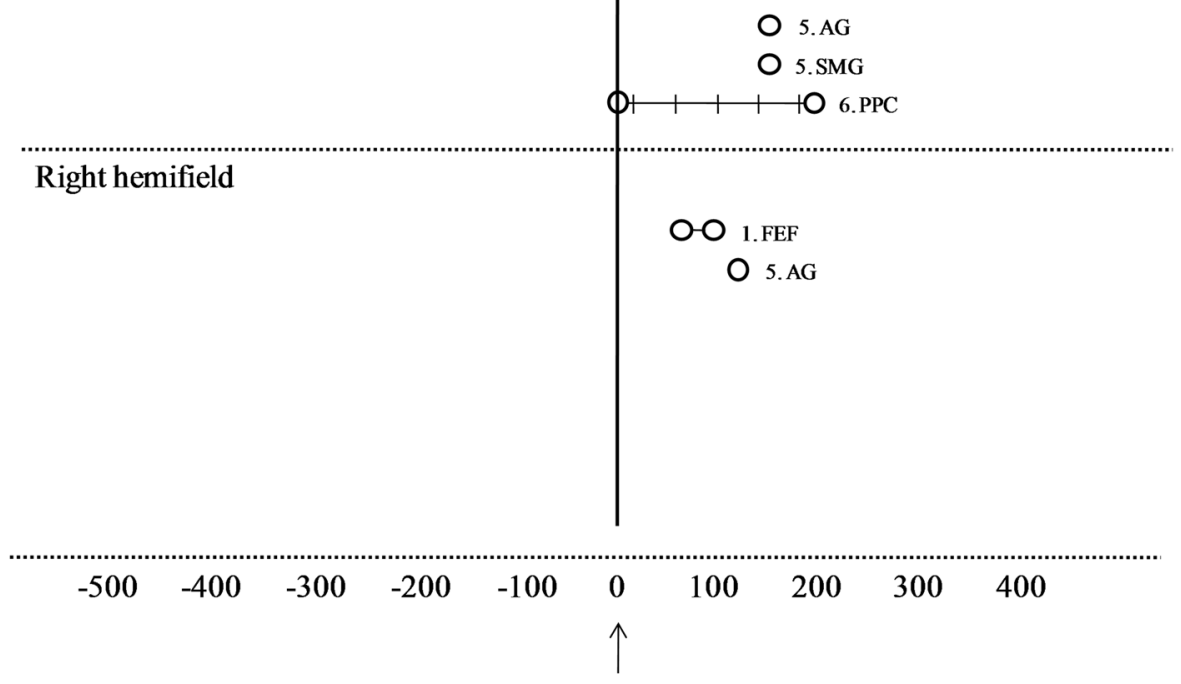

Time (ms)

Stimulus onset
Fig. 6 TMS parameters modulating direct visuo-motor pathway and cognitive control. The used TMS parameters were the following: 1.Bardi et al. 2012: paired pulse TMS (ppTMS) over left and right FEF (0-40, 40-80, 80-120, and 120-160 milliseconds (ms) after stimulus onset). 2.Bardi et al. 2015: single pulse TMS (spTMS) over left and right dPM $(100,130,160,190,220,250,280$, and 310ms after stimulus onset); 3.Herz et al. 2014: repetitive TMS (rTMS) over left and right pre-SMA $(1 \mathrm{~Hz})$ for 30' before task; 4.Praamstra et al. 1999: rTMS over left dPM (4

tendency. This figure shows left hemisphere dominance for both processes (i.e., direct visuomotor pathway and cognitive control).

Q4: concurrent activation: Feedforward and mutual inhibition. Some TMS studies provided evidence for parallel activation of the two competing responses (i.e., the spatial response and the response based on the task instructions). These studies applied TMS over the motor cortex while recording MEP from peripheral muscles in order to investigate muscular activity induced by TMS pulses (Stürmer, Siggelkow, Dengler, \& Leuthold, 2000; Van Campen, Keuken, van den Wildenberg, \& Ridderinkhof, 2014).

Stürmer, Siggelkow, Dengler and Leuthold (2000) delivered spTMS over left and right motor cortex $200 \mathrm{~ms}$ after stimulus presentation while MEPs were recorded from carpi pulses at $20 \mathrm{~Hz}$ ) at $300 \mathrm{~ms}-100 \mathrm{~ms}$ before stimulus onset. 5.Schiff et al. 2011: spTMS over left and right AG and SMG (70, 100, 130, 160, 190, 220, 250ms after stimulus onset); 6.Stürmer et al. 2007: rTMS (5 pulses at $20 \mathrm{~Hz})$ over left and right DLPFC (700-500ms and 500-300ms before stimulus), left and right dPM (300-100ms before stimulus), and left and right PPC (0-200ms after stimulus); 7.van Campen et al. 2018: rTMS over right IFG and pre-SMA $(1 \mathrm{~Hz})$ for $15^{\prime}$ before task

radialis muscle. In line with LRP studies, they obtained evidence for excitatory influence of the spatially compatible response since MEPs were larger in the incompatible condition than in the spatially compatible one. Van Campen, Keuken, van den Wildenberg and Ridderinkhof (2014) studied the inhibition of the spatially compatible response within the motor system by delivering spTMS over M1 at several times with regard to stimulus presentation. Motor excitability increased with time, starting earlier in the compatible than in the incompatible condition (as RT is faster in the compatible than in the incompatible condition) but an early and transitory increase in excitability was observed in the incompatible condition, which correlated with the number of errors. This increase of excitability strongly suggested the early influence of the response spatial tendency. These studies suggest concurrent activation of competing responses within the motor cortex.

Evidence for mutual inhibition of these competing responses was provided in a study conducted by Treccani, 
Cona, Milanese and Umiltà (2018). In this study, single-pulse TMS was applied over M1 $200 \mathrm{~ms}$ after stimulus presentation. The timing for applying the TMS pulse was chosen to align with the moment in which motor cortex activation is highest. Behavioural results showed the classical pattern elicited by sequential effects (i.e., the Simon effect was larger after compatible than after incompatible trials). MEP results showed that stimulus location activates an ipsilateral response (i.e., in the motor cortex primed by the stimulus location), which also inhibits the contralateral response. Also, activation of the spatially corresponding response was modulated by the previous trial. In trials preceded by compatible trials, MEP decreased in M1 ipsilateral (i.e., in the motor cortex primed by the stimulus location) for compatible trials and it increased for incompatible trials. For trials preceded by incompatible trials, MEP increased for M1 ipsilateral in compatible trials and it decreased in incompatible trials. The authors concluded from that evidence that reciprocal inhibition is a mechanism involved in response competition (Treccani, Cona, Milanese, \& Umiltà, 2018), as their experimental manipulations consistently showed that the covert activation of the spatially compatible response was accompanied by automatic inhibition of the contralateral M1.

Q6: Sequential congruency effects: Conflict adaptation and feature integration. Several TMS studies focused not only on the Simon effect but also on its modulation by the preceding trial, providing information about the brain areas playing a role in sequential effects and mechanisms leading to sequential effects. For instance, Stürmer, Redlich, Irlbacher and Brandt (2007) used repetitive TMS (rTMS) in a vertical Simon task. They found that rTMS over left DLPFC between 500 and $300 \mathrm{~ms}$ before stimulus presentation did not modulate the Simon effect itself but it reduced the sequential effects, suggesting that DLPFC has a role in context-dependent response control. This interpretation would be closer to feature integration than to conflict adaptation.

Studies reported evidence for motor activity associated with sequential effects even if trials did not require a motor response (Cona, Treccani, \& Umiltà, 2016). More specifically, these authors used TMS applied over M1 at $200 \mathrm{~ms}$ after stimulus presentation during a Go/No-go Simon task. Motor evoked potentials (MEPs) were used as dependent variable to study muscle excitability in response to the TMS pulse, which is reflecting the activity of the corticospinal pathway. They found that MEP activity was modulated by sequential effects even if the participant knew in advance that the upcoming trial would be a no-go trial. In light of this evidence, Cona, Treccani and Umiltà (2016) concluded that cognitive control may be automatically implemented and cannot only operate without awareness of the conflict but also without an intention to select a response (Desender \& Van den Bussche, 2012; van Gaal, Lamme, \& Ridderinkhof, 2010). Also, these results could be explained because integration of the stimulus features does not require intention to select a response. Altogether, TMS studies seem to be consistent with the episodic retrieval hypothesis to explain sequential effects.

\section{Functional magnetic resonance imaging (fMRI) studies}

Studies using fMRI were particularly useful to investigate specific brain areas activated during spatial conflict in the Simon task. In this section, we summarise the outcomes of a label-based analysis and a meta-analysis of the available fMRI studies investigating brain activity related to the Simon effect (for methods and results of the performed meta-analysis see Supplementary Information 2 (Online Supplementary Material). These approaches use the high spatial resolution of fMRI to provide information about specific brain areas involved in processing and resolving the spatial conflict, which is potentially useful to shed light on the third key question (Q3: Top-down cognitive control). We also provide a summary of fMRI studies that investigated sequential effects by using the Simon task, which may be related to the sixth key question (Q6: Sequential congruency effects: conflict adaptation and feature integration).

\section{Overview of previous fMRI studies in the Simon task and rationale for the performed analyses}

The first study to examine a Simon-like task in fMRI (Rosen et al., 1999) used a task variant with the so-called endogenous cueing using central arrow cues and compared both orienting conditions (valid and invalid) with the resting condition. As we intend to characterise brain regions involved in conflict processing during the Simon task, we however only considered contrasts without the valid in the present review. Accordingly, the chronologically first study included in this review was conducted in 2001 (Maclin, Gratton, \& Fabiani, 2001). The majority of subsequent studies on Simon effects investigated not only the Simon task but also incorporated other factors into their experimental designs, like additional conflicting dimensions (e.g., Jiang \& Egner, 2014; Kim, Johnson, \& Gold, 2012; Korsch, Frühholz, \& Herrmann, 2014; Wittfoth, Schardt, Fahle, \& Herrmann, 2009), or compared healthy controls with a patient group (e.g. GeorgiouKaristianis et al., 2012; Schmitz et al., 2006; Sebastian et al., 2012).

Former meta-analyses including Simon tasks and using fMRI data examined broader topics (e.g., spatial interference, see Cieslik, Mueller, Eickhoff, Langner, \& Eickhoff, 2015; stimulus-response conflicts, see Li et al., 2017; interference resolution, see Nee, Wager \& Jonides, 2007, and Zhang, Geng \& Lee, 2017; non-emotional spatial interference processing, see Xu, Xu, \& Yang, 2016). The number of analysed Simon 
studies in these meta-analyses varied from four (Nee, Wager \& Jonides, 2007) to 23 (Li et al., 2017), and none of them reported results separately for the Simon task. Thus, whereas former meta-analyses on fMRI data examined conflict processing in a broader way, this is the first meta-analysis that exclusively focuses on data from Simon tasks. This approach provides useful information about brain areas activated during the Simon conflict, which sheds light on key question 3 . In the following, we present the methods and results for the labelbased analysis, and then summarise and discuss results from the activation likelihood estimate (ALE) meta-analysis (Eickhoff et al., 2009; Eickhoff et al., 2012) carried out by using the coordinates of all included studies (see Supplementary Information S2 (Online Supplementary Material)). Given the multidimensional nature of fMRI data, the meta-analysis is an appropriate approach to integrate information from all the available studies. However, we did not adopt a meta-analytical approach for key question 6 due to the low number of fMRI studies investigating brain activity related to sequential congruency effects in the Simon task.

\section{Label-based analysis: Methods}

We only included fMRI data from adult participants, excluding data from children or adolescents. Samples only consisting of older participants ( $>60$ years) or patients were also excluded. Furthermore, to characterise areas involved in Simon interference, only studies reporting contrasts related to the incompatible condition are reported. Li et al. (2017) reported activity in similar brain regions when using incompatible trials contrasted with compatible trials compared to incompatible trials contrasted with a neutral baseline. We included one study using an incompatible > neutral contrast (Forstmann, van den Wildenberg, Wery, \& Ridderinkhof, 2008a) and one study reporting a main effect of the incompatible condition (Forstmann et al., 2008b), while all other studies used the incompatible $>$ compatible contrast. One study did not find any activation related to the Simon interference (Peschke et al., 2016). As the Simon interference should be assessed with fMRI throughout the whole brain, all studies solely examining specific regions of interest (ROIs) or using a restricted search volume that did not provide data on a whole brain level were excluded. One study (Wittfoth, Buck, Fahle, \& Herrmann, 2006) examined two variants of the Simon task with the same sample in one scanning session (counterbalanced order). To prevent a double weighting of this particular sample, only the data from the standard (location-based) Simon task were used here, while the data from the motion-based task were not included. Studies describing activated brain areas without delivering stereotactic coordinates in either Montreal Neurological Institute (MNI) or Talairach space were excluded, which left data from 25 studies meeting these criteria (see Supplementary Information 3, 4, and 5 (Online Supplementary Material)).

The label-based approach was applied summarising all the labels of all activated regions of the 25 Simon studies into a common table (Supplementary Information 3 and 6 (Online Supplementary Material)). For all but one study (Sommer, Hajak, Döhnel, Meinhardt, \& Müller, 2008) the labels were extracted from tables. Sommer and colleagues provided their labels and corresponding coordinates within the text. In cases where more than one label was assigned to a cluster characterising the activated regions all these labels were used. Only when labels were too general (e.g., temporal gyrus, lentiform nucleus) or too many labels were provided for small clusters (e.g., middle occipital gyrus/superior occipital gyrus/ superior parietal lobule for a cluster with 42 voxels, see Van Eijk et al., 2015), we looked up the exact brain area at the peak coordinate (using the Talairach daemon client, Lancaster et al., 2000) and used this label instead. In a few cases, this procedure led to the circumstance that a region is specified in the table to be activated in a study even if this region was not mentioned in the corresponding publication. When coordinates had been reported in Talairach space, we transferred the coordinates to MNI space with GingerALE (GingerALE 2.3.6, http://brainmap.org/ale/) using the correct transformation algorithm dependent on the software package used for fMRI data analysis in the corresponding publication. To extract key structures involved in Simon conflict we only considered brain regions generally activated in more than five studies ( $=$ in at least $24 \%$ of studies, irrespective of activated hemisphere).

\section{Label-based analysis: Results}

When summarising the regions reported in the 25 Simon studies (see Supplementary Information 4 (Online Supplementary Material) for a summary of the label-based results), the most commonly activated region was the inferior parietal lobule (IPL), fairly balanced between hemispheres $(9 \times$ activity in right IPL, $11 \times$ in left IPL, thereof $7 \times$ bilateral IPL). This was also the region showing the most bilateral clusters. The next most frequently activated regions were precuneus and middle frontal gyrus (MFG). MFG activity also was relatively evenly distributed between hemispheres $(8 \times$ right, $7 \times$ left hemisphere, thereof five bilateral clusters). Activation of the precuneus was slightly more frequent in the left hemisphere ( $9 \times$ left, $6 \times$ right hemisphere, thereof three bilateral clusters). Superior frontal gyrus (SFG), insula, and inferior frontal gyrus (IFG) were activated in both hemispheres (SFG: $8 \times$ right, $6 \times$ left hemisphere; insula: $6 \times$ right, $8 \times$ left hemisphere; IFG: $7 \times$ right, $6 \times$ left hemisphere). Whereas this also held true for SPL (7× right, $6 \times$ left hemisphere), activity in superior temporal gyrus showed a clear tendency to the right hemisphere $(9 \times$ right, $4 \times$ left hemisphere). Activity in middle temporal gyrus 
(MTG) showed the same pattern with a right-hemispheric focus $(9 \times$ right, $3 \times$ left hemisphere $)$. Active clusters in precentral gyrus and caudate nucleus were evenly distributed across hemispheres $(5 \times$ in each hemisphere, bilateral clusters in three cases each). Further common activity across Simon tasks was found in bilateral thalamus (five right, four left hemisphere, bilateral clusters in three cases) and in anterior cingulate cortex (ACC) mainly in the left hemisphere $(2 \times$ right, $6 \times$ left hemisphere). Cingulate gyrus was reported to show activity in seven Simon studies (including activity in posterior and middle cingulate gyrus) and activity in supplementary motor area (SMA) was reported in six studies (SMA: $4 \times$ left, $1 \times$ right hemisphere, $1 \times$ medial) with an emphasis on left-hemispheric activation.

\section{Summary of fMRI meta-analytical results}

The ALE analysis yielded four clusters (see Supplementary Information 2 and Supplementary figure (Online Supplementary Material)): A parietal cluster extending from right SPL (superior parietal lobule) to IPL and precuneus; a medial frontal cluster comprised medial frontal and cingulate gyri (SMA/pre-SMA); a posterior cluster showed voxels in right MTG, inferior temporal and middle occipital gyri; and the last cluster comprising the left middle frontal and precentral gyri. In the following, the findings of the ALE meta-analysis are compared to former meta-analytic outcomes from other studies. Then, the results of the label-based analysis and the meta-analysis are integrated. Subsequently, potential functions of the involved brain areas are outlined.

In former meta-analyses including Simon tasks, broader topics were examined and no separate effects were reported for the Simon task. And yet, the reported effects are located in brain areas comparable to those we found. For example, right SPL was also reported in three other meta-analyses (Cieslik, Mueller, Eickhoff, Langner, \& Eickhoff, 2015; Li et al., 2017; $\mathrm{Xu}, \mathrm{Xu} \&$ Yang, 2016) and right IPL in two (Nee, Wager, \& Jonides, 2007; Xu, Xu, \& Yang, 2016). Activity in (pre-)SMA and/or medial frontal/ACC overlapping with our coordinates and close to our cluster in medial frontal gyrus was found in all other meta-analyses on interference processing referred here (Cieslik, Mueller, Eickhoff, Langner, \& Eickhoff, 2015; Nee, Wager, \& Jonides, 2007; Li et al., 2017; Xu, Xu, \& Yang, 2016; Zhang, Geng, \& Lee, 2017). The present cluster in left $\mathrm{MFG} /$ precentral gyrus was located near two clusters in MFG and precentral gyrus from other meta-analyses (MFG: Cieslik, Mueller, Eickhoff, Langner, \& Eickhoff, 2015; precentral: Zhang, Geng, \& Lee, 2017). These meta-analyses also reported corresponding clusters in the right hemisphere, together with $\mathrm{Xu}, \mathrm{Xu}$ and Yang (2016) who only found a righthemispheric cluster. The cluster in right MTG showed no correspondence to results of other meta-analyses. Only $\mathrm{Xu}$,
$\mathrm{Xu}$ and Yang (2016) reported activity in MTG, although their cluster was located in the left hemisphere.

In sum, the ALE analysis produced clusters that are in line with the results of other, less specific meta-analyses that included Simon tasks. Only for the cluster in MTG was there no matching region in other studies (only one in the other hemisphere in the study of $\mathrm{Xu}, \mathrm{Xu}, \&$ Yang, 2016).

\section{Integration of label-based and meta-analytic analyses}

Both approaches (ALE meta-analysis and label-based) showed IPL as an important area involved in Simon interference. Whereas the label-based data showed a rather balanced ratio between hemispheres for IPL activity, the ALE metaanalysis only yielded a cluster in right IPL extending to SPL and precuneus. Indeed, in the label-based analysis, activity in precuneus showed a slight bias towards the right hemisphere, whereas SPL was activated similarly in both hemispheres. Our ALE cluster in medial frontal and cingulate gyri including the transition zone between SMA and pre-SMA showed a peak in the left hemisphere. This is in good correspondence with the mainly left-hemispheric activities in SMA and ACC found in our label-based analysis. Activity in MFG and precentral gyrus was captured by a left-hemispheric ALE cluster, whereas the label-based analysis points at a rather bilateral distribution, especially for the MFG (five cases with bilateral activity). The ALE cluster in right MTG is in line with the fact that MTG activity in the label-based analysis was primarily observed in the right hemisphere.

Further brain areas not detected with the ALE analysis that were activated in at least six of the 25 Simon studies comprised bilateral SFG, IFG, and insula, superior temporal gyrus (mainly in the right hemisphere, comparable to the MTG lateralisation), bilateral caudate nucleus, and bilateral thalamus.

\section{Potential functions of activated brain areas}

Posterior parietal cortex particularly in the right hemisphere has already been linked to stimulus-response compatibility (Li et al., 2017; Nee, Wager, \& Jonides, 2007). Furthermore, SPL was reported to be involved in spatial attention and especially the relocation of spatial attention (Molenberghs, Mesulam, Peeters, \& Vandenberghe, 2007; Yantis et al., 2002). In 21 of the 25 analysed Simon studies there was a target stimulus displacement inducing shifts of spatial attention. Li et al. (2017) found left superior parietal cortex to be more engaged in stimulus-stimulus conflict processing and the corresponding right-hemispheric region to be more involved in the processing of stimulus-response conflict. In line with the finding of a right-hemispheric ALE cluster, stimulus-response conflict is present in all Simon tasks. Additionally, many of the analysed task variants contained stimulus-stimulus conflicts. 
This might account for the rather bilateral activity of the labelbased results.

The ALE cluster in medial frontal and cingulate gyri comprised the overlapping zone between pre-SMA and SMA regions (Mayka, Corcos, Leurgans, \& Vaillancourt, 2006). The label-based analysis yielded further activity in cingulate areas, ACC, and SMA. Pre-SMA has been associated with response conflict-related activity (Nachev, Husain, \& Kennard, 2008), successful inhibitory control (Chen, Muggleton, Tzeng, Hung, \& Juan, 2009), and initiating or selecting action sets (Rushworth, Walton, Kennerley, \& Bannerman, 2004). In our results, the side of (pre-) SMA activity was not unequivocally associated with the side of response hand as five of the six studies contributing to this ALE cluster used button presses with the left and right index finger instead of a right-lateralised response. A region comprising medial cingulate cortex as well as parts of the SMA was suggested to be part of the socalled multiple demand network (Müller, Langner, Cieslik, Rottschy, \& Eickhoff, 2015). These authors criticise that in previous studies this region was often misleadingly labelled 'dorsal ACC' and suggest using the label 'MCC/SMA' (midcingulate cortex/pre- and SMA) instead. ACC has been linked to different kinds of functions and concepts in the past (see Bush, Luu, \& Posner, 2000; Weston, 2012). Taken altogether, this region seems to be involved in reinforcement learning processes and in different contextspecific supervisory and evaluative processing mechanisms that (on a trial-by-trial basis) assess behavioural performance (see Mansouri, Egner, \& Buckley, 2017).

Our ALE cluster with voxels in right MTG, inferior temporal gyrus, and middle occipital gyrus showed a $23 \%$ probability for area V5/MT (middle temporal visual area) according to the Anatomy toolbox. Interestingly, two of the five studies contributing to this cluster used moving dot stimulation (Wendelken, Ditterich, Bunge, \& Carter, 2009; Wittfoth, Buck, Fahle, \& Herrmann, 2006). This might point to a specific involvement of areas drawn upon in a particular task variant. Task-specific enhanced activity in this region might be caused by attentional enhancement during the more demanding incongruent condition, as MT+ is known to be modulated by attention (O'Craven, Rosen, Kwong, Treisman, \& Savoy, 1997; Treue \& Maunsell, 1996). Nevertheless, when looking at activity in MTG without focusing on area MT+, the label-based analysis showed activity in ten studies in this region. Superior temporal gyrus was activated in 11 studies in the label-based analysis. Two of these studies involved auditory stimulation but the other studies employed diverse visual stimulation (from colour drawings to arrows or simple shapes). These results indicate that middle and superior temporal areas lacking in former meta-analyses on conflict processing (apart from left MTG in Xu, Xu, \& Yang, 2016) are indeed involved in Simon tasks.
A cluster in left MFG/precentral gyrus was found in the ALE analysis and bilateral activity in precentral gyrus and in MFG in the label-based analysis. Given that former metaanalyses reported bilateral activity in these areas (Cieslik, Mueller, Eickhoff, Langner, \& Eickhoff, 2015; Zhang, Geng, \& Lee, 2017), we consider our label-based findings consistent with the overall picture. Possibly, activity in right MFG/precentral gyrus was too distributed across studies to produce a corresponding cluster in the ALE analysis as well. Activity in right MFG has been linked to interference resolution (e.g., Tang, Critchley, Glaser, Dolan, \& Butterworth, 2006). However, it might not be necessary to actively resolve conflict in the Simon task, given that a study perturbating right MFG with transcranial magnetic stimulation (TMS) found no effects on the Simon effect in a double-conflict task (Peschke et al., 2016). Indeed, their targeted region was located more anterior and inferior compared to our results. In fact, most of the studies showing MFG activity in the label-based analysis reported MFG coordinates located considerably more anterior and inferior (mostly BA 9, some in dorsolateral prefrontal cortex, DLPFC) than our MFG ALE cluster coordinates (BA $6)$.

Activity in caudate nucleus has been linked to contingencies between actions and their consequences (Grahn, Parkinson, \& Owen, 2008) and to higher levels of stimulus ambiguity (Provost, Hanganu, \& Monchi, 2015). The caudate nucleus seems to participate in response inhibition processing via two pathways: a hyperdirect one (fronto-subthalamic) and an indirect one (fronto-striatal-pallidal; Jahfari et al., 2011).

Some of the regions found in our analyses form part of the so-called 'multiple-demand system' suggested by Duncan (2010) that is involved in different executive processing mechanisms (working memory, attention, inhibition; Müller, Langner, Cieslik, Rottschy, \& Eickhoff, 2015). The regions reported by these authors comprise mid-cingulate/SMA, right inferior parietal cortex, bilateral inferior frontal and (anterior) insular cortex, as well as right MFG (our ALE cluster was located in left MFG but the label-based analysis yielded bilateral MFG activities). Bilateral insular and IFG activation are also reported to be involved in conflict processing in metaanalyses including Simon tasks (Cieslik, Mueller, Eickhoff, Langner, \& Eickhoff, 2015; Nee, Wager, \& Jonides, 2007; Li et al., 2017; Xu, Xu \& Yang, 2016; Zhang, Geng, \& Lee, 2017).

\section{Possible limitations of the performed analyses}

It should be noted that the studies included in our analyses showed heterogeneous design characteristics even if they all assessed the Simon effect. There were differences in experimental design parameters, types of stimuli, and sample characteristics (e.g., gender distribution). 
Different studies used distinct probabilities for the contrasted conditions. However, areas in parietal, frontal, and temporal areas as well as in bilateral thalamus are reported to show a higher level of activity for the Simon conflict when a higher proportion of incongruent stimuli are used (75/25\% compared to $25 / 75 \%$; Xia, Li \& Wang, 2016). Thus, differing stimulus frequencies might have influenced the results of the individual studies and accordingly our results. Additionally, various timings were used for the succession of Simon stimuli. Some studies included null trials to allow the hemodynamic response to return to baseline. Considerably differing stimuli with varying characteristics were used throughout the studies ranging from rather commonly used arrow stimuli to face stimuli (e.g., Jiang and Egner, 2014). The lateral stimulus displacement was larger or smaller in different studies and absent in other studies (using central arrow stimuli or a motion-based task variant with central stimulation). Most studies additionally examined other factors influencing the Simon effect. Thus, the overall number of conditions within the experimental design varied between tasks and some studies incorporated conditions with neutral stimuli, other types of conflict, different emotional expressions and so on. This fact might also have influenced neuronal responses in the analysed incompatible and compatible conditions. Some studies tried to control for sequential modulations of the Simon effect: for example they only reported data of trials following compatible trials (a method that might prevent control adjustments but does not prevent feature-integration effects), whereas other studies seemed to use all trials regardless of the preceding trial type. Most of the studies included both sexes but three studies only examined male samples (Page et al., 2009; Schmitz et al., 2006; Sommer, Hajak, Döhnel, Meinhardt, \& Müller, 2008) and one only female participants (Van Eijk et al., 2015). Unequal gender distributions might influence the results as women are reported to show higher Simon effects and stronger post-error slowing (Stoet, 2017).

Another caveat of our label-based comparison of the 25 analysed Simon studies is that it depends on the correct labelling of the regions. With this approach we have to rely on the labels the authors assigned to their clusters and hope that they were actually representative of the clusters (and not just identify a peak that might be located at the border area of the cluster with only a few voxels lying in this anatomical region). To complement our data, we also provided results of an ALE meta-analytic approach. In this approach only peak coordinates are taken into consideration. However, they might not constitute the best representation of the corresponding activated brain regions. Brain regions might not show up in the ALE analysis when different parts of these regions are activated in different studies. Accordingly, we argue that a broader perspective of brain regions involved in Simon task processing requires the consideration of both label-based and ALE analyses - so to compensate for the weaknesses of both methods.

\section{fMRI and key questions}

Q3: Top-down cognitive control: Support of the correct response versus inhibitory control mechanisms. In general, findings of increased fMRI activity in incompatible than compatible conditions are taken to reflect conflict processing and resolution, perhaps involving the activation of dedicated inhibitory control systems. It is important to point out that other interpretations are possible, as the increased activation may just as well indicate mere monitoring or recording of conflict, as for instance other cognitive activities need to stop until the conflict resolves itself, or to signal the need to screen the context for indicators correlated with the conflict and to trigger learning processes integrating these indicators (so as to better predict such conflict in the future). In any case, the metaanalysis conducted in the present study showed the existence of four clusters of activity associated with the Simon effect, which also were quite consistent with activity revealed by the label-based analysis; specifically, two clusters were observed in frontal regions (middle cingulate cortex and medial frontal cortex; left middle frontal gyrus and left precentral gyrus), one cluster was observed in parietal areas (right superior and inferior parietal lobes) and another one in occipitotemporal regions (middle and inferior temporal gyri and middle occipital lobe).

Increased neural activity in frontal and parietal areas is consistent with the existence of a frontoparietal network involved in detecting and resolving the conflict. In this regard, Sebastian et al. (2013) proposed two participating networks to support and inhibit the response during the performance of a response inhibition paradigm. The first network comprising frontoparietal and premotor regions is engaged in response selection and the second, a frontostriatal network including right inferior frontal cortex and caudate nucleus (which showed also a significant activation in the present analyses), is involved in the implementation of response inhibition. According to the present data, these networks seem to be involved in Simon task processing and might be related to response selection - fronto-parietal network - and response inhibition - fronto-striatal network. In the Summary and integration section, we have put together findings from fMRI, EEG/ERP and TMS, which provides a better angle to address the question of whether the areas reported by the meta-analyses and label-based analyses may be more involved in supporting and/or inhibiting the response.

Finally, the present data demonstrated that middle and superior temporal areas, particularly in the right hemisphere, also participate in the Simon conflict. Even if we hypothesise in the next section about some specific functions that may be associated with this neural activation (i.e., augmented 
processing of stimulus features may be needed in trials containing conflicting spatial information), further research is necessary to elucidate the specific role of these regions in conflict processing.

Q6: Sequential congruency effects: Conflict adaptation and feature integration. There are only a few fMRI studies examining sequential effects in the Simon task. In the study of Kerns (2006), preceding conflict monitoring processes expressed in higher anterior cingulate cortex (ACC) activity were found for sequential effects with subsequent higher activity in prefrontal cortex (PFC). Furthermore, the higher the ACC activity on the preceding trial the more PFC activity was present on the subsequent trial. The authors discuss the possibility that conflict adaptation and feature integration might have interacted to generate these results. In contrast, Egner (2007) took the findings of Kerns (2006) to speak against the feature integration hypothesis, as feature integration should have resulted in lower PFC activity on fast i-I trials due to more priming whereas Kerns (2006) observed higher PFC activity on fast i-I trials. However, Egner's (2007) interpretation should be taken cautiously, because the relationship between frontal activity and task difficulty may follow an inverted U-shape function. Hence, if the task difficulty is low, increasing the difficulty might lead to increased frontal activation; however, if the task difficulty reaches the medium level, further increasing the difficulty might reduce the activation. It would thus be essential to determine whether the fast i-I trials under analysis are related to low or medium difficulty. Unfortunately, the experimental design used by Kerns (2006) does not allow answering this question. Additional evidence for a role of feature integration comes from a recent study showing sequential congruency effects in visuomotor brain regions (left precentral gyrus, left central sulcus, left postcentral gyrus, left striate cortex, right cerebellum) related to the updating of stimulus-response bindings (Aisenberg, Sapir, Close, Henik, \& d'Avossa, 2018).

Egner, Delano and Hirsch (2007) used a factorial combination of trials eliciting Stroop and Simon conflicts and found sequential effects for both types of conflict. An interesting finding was the fact that both sequential effects showed no correlation within participants. Furthermore, sequential effects led to activity in bilateral precentral gyri under Simon conflict whereas activity in precuneus/superior parietal lobule was observed under Stroop conflict. The authors interpret the data as pointing to the existence of two different conflict-specific control systems which, as they argue, might be hard to reconcile with the assumption of a single underlying mechanism like episodic memory (feature integration hypothesis). However, different and separable types of neural activation may reflect differences in the loci or format of the feature codes being integrated rather than any difference in the mechanism responsible for the integration.

Horga et al. (2011) reported increased activity with increasing level of conflict (i.e., the experimental conditions, from the lowest to the highest level of conflict, were the following: c-C, $\mathrm{i}-\mathrm{C}, \mathrm{i}-\mathrm{I}, \mathrm{c}-\mathrm{I})$ in a left-hemispheric area spanning pre-SMA and dorsal ACC, a portion of the right precentral gyrus (including parts of the frontal eye field, FEF), and right inferior frontal junction (IFJ). The activity pattern in pre-SMA and IFJ revealed higher activity for trials with a congruence alternation from one trial to the next (i.e., i-C and c-I trials). Pre-SMA was also involved without conflicting information, as pre-SMA activity was larger in i-C than c-C trials. Blank trials (long inter-trial intervals) were used to assess preparatory activity with regard to the previous trial type. Preceding conflict led to higher activity on blank trials in left dorsomedial PFC, left angular gyrus and left precuneus. Furthermore, the activity pattern in dorsomedial PFC reflected trial history. The more conflict trials had been presented previously, the higher the activity in dorsomedial PFC. Furthermore, a higher number of consecutive congruent trials led to reduced activity in dorsomedial PFC. An association with RT confirmed that the increasing preparatory activity in dorsomedial PFC indeed predicted a speed-up of RTs in the upcoming incongruent but not upcoming congruent trials. The authors proposed that the two systems might interact to enable the flexible adaptation during sequential effects. The first system is active in response to the stimulus and probably involved in conflict processing (pre-SMA, dorsal ACC, right precentral gyrus, right IFJ), whereas the second system is a preparatory mechanism and codes conflict history (rostral dorsomedial PFC, left angular gyrus, left precuneus).

Whereas the study of di Pellegrino, Ciaramelli and Làdavas (2007) did not use fMRI, their results can add important knowledge concerning brain areas crucial for the processing of sequential effects. They examined patients with focal lesions either within rostral ACC (and adjacent ventromedial PFC, eight participants), patients with focal lesions outside the PFC, and a healthy control group. Only the patients with lesions in rostral ACC did not show an effect of sequential modulation in their behavioural data. Their Simon effects remained comparable regardless of the preceding trial (even when trials with direct repetitions of stimuli or responses were excluded), indicating an important role of rostral ACC/ ventromedial PFC involvement in the processing of sequence effects.

It is worth mentioning two fMRI studies that illustrate the importance of learning contingencies in the Simon task. Xia, $\mathrm{Li}$ and Wang (2016) reported that reversing the Simon effect by increasing the proportion of congruent trials was accompanied by higher anterior middle cingulate cortex (aMCC) and 
DLPFC activity in the compatible as compared to the incompatible condition, which might indicate the importance of the context (learning of S-R associations) in the emergence of the Simon effect. Importantly, conflict adaptation cannot explain these data: a given participant may well upregulate control to a level that eliminates conflict, thus bringing the Simon effect to zero, but this should not result in faster RTs to incompatible than compatible trials. Similarly, Li, Xia and Wang (2015) used the Hedge and Marsh (1975) paradigm, which requires responding to the opposite colour that appears on the screen. Findings showed a reversed Simon effect and higher aMCC and DLPFC activity in the compatible as compared to the incompatible condition, suggesting that cognitive control was stronger in the compatible than the incompatible condition.

It should be noted that there are no specific neural areas showing consistence across the different studies (precentral gyrus, ACC, left prefrontal areas). This becomes apparent when comparing the distances between locations reported in different studies for the same anatomical areas. These differences lie in the order of several centimetres, in one case even almost $7 \mathrm{~cm}$ from each other (locations in prefrontal cortex from Kerns, 2006 compared to those of Horga et al., 2011). Although further studies are required, these differences might be related (at least partially) to differences in the experimental design.

In short, there are some fMRI studies interpreting the obtained data in line with conflict adaptation (Egner, Delano \& Hirsch, 2007; Horga et al., 2011), whereas others suggest that feature integration is the mechanism underlying sequential modulations of the Simon effect (Aisenberg, Sapir, Close, Henik, \& d'Avossa, 2018; Li, Xia, \& Wang, 2015; Xia, Li, \& Wang, 2016). Also, some researchers explicitly suggested that conflict adaptation and feature integration may interact to produce sequential effects (Kerns, 2006). Evidence for both mechanisms may be in agreement with the existence of conflict adaptation and feature integration simultaneously occurring at different levels of abstraction (see Abrahamse, Braem, Notebaert, \& Verguts, 2016; Egner, 2014). Alternatively, these findings may be also illustrating the difficulty to completely prevent integration of S-R features in the experimental designs (Spapè \& Hommel, 2014) and/or the possibility that adaptation is achieved through feature integration (Spapé \& Hommel, 2008; Dignath et al., 2019).

\section{Summary and integration}

Let us now try to integrate the reviewed neurophysiological findings and discuss to what extent they shed light on the theoretical questions previously formulated. Figure 7 schematically recaps whether and to what extent neurophysiological research provides consistent support for theoretical accounts, hypotheses, and explanatory mechanisms summarised in the key questions.

\section{Q1: Early versus late locus of the Simon effect}

In the experimental and neuroscientific Simon studies from 1970s and 1980s, identifying the locus of the effect was the major aim. Electrophysiological studies used the LRP component to demonstrate that the Simon effect is related to a delay in the selection of the correct response. These studies showed a transient and sub-threshold motor activity in the hemisphere contralateral to the stimulus location in incompatible trials, which was systematically related to a delay in the preparation of the correct response (e.g., De Jong, Liang, \& Lauber, 1994; Stürmer, Ouyang, Zhou, Boldt, \& Sommer, 2013; ValleInclán, 1996). There are no TMS studies specifically designed to investigate the locus of the Simon effect, but demonstrations of a transient increase of motor excitability in the hand ipsilateral to the stimulus location in incompatible trials (Stürmer, Siggelkow, Dengler, \& Leuthold, 2000; Van Campen, Keuken, van den Wildenberg, \& Ridderinkhof, 2014) suggest a transitory (and subthreshold) preparation of the response primed by the stimulus location. Accordingly, neurophysiological research consistently showed evidence for a late locus of the Simon effect (that is, in the selection of the response), as stated by dual-route models (De Jong, Liang, \& Lauber, 1994; Kornblum, Hasbroucq, \& Osman, 1990; Proctor \& Vu, 2006; Wascher, Schatz, Kuder, \& Verleger, 2001). At the same time, the available findings speak against an early locus of the Simon effect, as proposed by perceptual models (Hasbroucq \& Guiard, 1991).

Still open is the question of why compatibility effects are sometimes larger in stimulus-locked LRPs than in RTs (Stürmer, Ouyang, Zhou, Boldt, \& Sommer, 2013). Some authors have attributed these observations to the possibility that faster response execution in incompatible rather than compatible trials may reduce the effect in RT but not in LRP (Cespón, Galdo-Álvarez \& Díaz, 2013a; Cespón, GaldoÁlvarez, \& Díaz, 2013b; Shang, Fu, Qiu, \& Ma, 2016) and others have held contamination from the N2cc waveform responsible (Stürmer, Ouyang, Zhou, Boldt, \& Sommer, 2013). Future research will be necessary to disentangle these possibilities.

\section{Q2: Early versus late formation of spatial codes}

Given the functional and evolutionary plausibility of synergies between attention and action control, a substantial number of studies using the Simon task was devoted to studying such synergies.

Several ERP studies showed an association between attentional shifts (as indicated by the N2pc) and activation of the spatial response (as revealed by the Simon effect and LRPs), 


\section{Neurophysiological evidence for the theoretical issues}

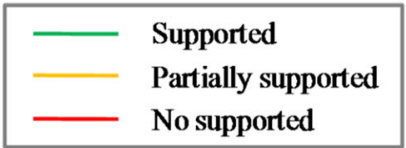

Q1: Early vs. late locus of
the Simon effect $\left\{\begin{array}{l}\text { Perceptual conflict } \\ \text { Selection of the response }\end{array}\right.$

ERP: LRP

Q4: Concurrent activation:
feedforward and/or mutual inhibition
ERP: LRP
ERP: Laplacian analysis showed no anticorrelation between motor cortices
TMS over M1 and anticorrelated MEP activity in both hands

Q5: Temporal overlap of the $\left\{\begin{array}{l}\text { Strength-difference models } \\ \text { activated responses }\end{array}\right.$ Time-difference models

ERP modulations according to time of processing of relevant and irrelevant stimulus features

\section{Q3: support of the correct response and/or inhibitory control mechanisms \\ ERP: Laplacian analysis over both motor cortices \\ EEG: theta power modulations \\ TMS over areas related to cognitive control (see Figure 6) \\ fMRI: frontal and parietal clusters of activity related to Simon conflict}

Fig. 7 Summary of the neurophysiological evidence for the theoretical issues. This figure represents to what extent neurophysiological studies provided evidence for or against theoretical models, accounts, and mechanisms proposed to explain different issues related to the Simon task research. "Supported" refers to a majority of results being

which was taken as evidence for the PMTA (Van der Lubbe, Abrahamse, \& De Kleine, 2012). However, given that PMTA as well as attentional shift accounts predict an association between attentional shift and Simon effect, studies investigating the temporal relationship between correlates of attention and action (for instance, N2pc and LRP, respectively) would be important to confirm whether the formation of spatial codes occurs in parallel to or later than response activation. As far as we know, there are no ERP studies specifically designed for such purpose, even if research using N2pc and LRP suggests that both components take place in the same time window. Similarly, TMS studies using the Simon task suggested that attentional and motor processes may overlap with respect to brain regions - FEF (Bardi, Kanai, Mapelli, \& Walsh, 2012) and PPC (Schiff, Bardi, Basso, \& Mapelli, 2011) - and time window, which is also consistent with other studies reporting close relationships between attention and action (Andersen \& Buneo, 2002; Eimer, Forster, Van Velzen, \& Prabhu, 2005;

\section{Q6: Conflict adaptation
and/or feature integration $\left\{\begin{array}{l}\text { Comfllicit and anplatiliom } \\ \text { Feature integration }\end{array}\right.$}

ERP: specific modulations of cognitive control (N2) and switching (P3)

EEG: experimental designs and modulation of theta power

TMS over DLPFC and motor areas

fMRI: experimental designs preventing feature integration or conflict adaptation

consistent with that view; "Partially supported" indicates that some results support that view or mechanism but they are far from being consistent; "No supported" denotes that available research does not support that view, model, hypothesis, or explanatory mechanisms

Eimer, Van Velzen, Gherri, \& Press, 2007; Sereno \& Huang, 2014). Overall, even if additional research will be needed, these results suggest that spatial encoding and response activation occur at about the same time, as assumed by late attentional accounts, that is, PMTA (Sheliga, Craighero, Riggio, \& Rizzolatti, 1997) and referential coding account (Hommel, 1993).

There is also behavioural and ERP evidence showing that an attentional shift may be neither necessary nor sufficient to activate the direct visuo-motor pathway and give rise to the Simon effect, which suggests that spatial codes may be formed in the absence of, and thus independently from attentional shifts. In particular, an attentional shift may be not needed to give rise to the Simon effect, as revealed by several studies that reported Simon effects in RT and LRP with monocular presentation of the stimuli in participants who were unaware of the stimulated eye (Valle-Inclán, Hackley, \& De Labra, 2003; Valle-Inclán, Sohn, \& Redondo, 2008). These 
studies suggest the existence of spatial encoding without attentional shifts, which fits with the referential coding account (Hommel, 1993). Even so, it may be argued that, in those mentioned studies, attentional shifts could have been unconsciously produced (Sato, Kochiyama, Uono, \& Toichi, 2016), preserving the validity of PMTA to explain those results. Interestingly, a later study by Schankin, Valle-Inclán and Hackley (2010) reported that the Simon effect may result from either of two separate mechanisms: the formation of spatial codes mediated by attentional shifts or the formation of spatial codes without attentional shifts.

Another study dissociating attention and action was reported by Valle-Inclán and Redondo (1998), who showed that a lateralised stimulus did not produce automatic activation of the ipsilateral response - as revealed by LRP measures - when the response assigned to each relevant feature of that stimulus was still unknown at the time of its presentation. Thus, having information about $\mathrm{S}-\mathrm{R}$ ensembles is mandatory to activate the direct visuo-motor pathway, which fits with the referential coding account and undermines accounts arguing that stimulus-response compatibility relies on automatic processes (e.g., Kornblum et al., 1990; see Hommel \& Wiers, 2017). In this regard, an fMRI study demonstrated that preparing a specific action increases the activation of brain areas that are coding perceptual consequences related to the intended action (Kühn, Keizer, Rombouts, \& Hommel, 2011), which argues for common functional and neural representations of stimulus and response features. The importance of stimulus and response association was also highlighted by $\mathrm{Cao}$, Cao, Yue and Wang (2017), who reversed Simon effects in behavioural and electrophysiological measures (i.e., increased N200 and decreased P300 in the compatible condition as well as higher theta power in mid-frontal regions) by using a proportion congruency paradigm. Another consistent finding was reported by Li, Xia and Wang (2015; Xia, Li, \& Wang, 2016), who observed behaviourally and neurally reversed Simon effect by using the Hedge and Marsh paradigm (Hedge \& Marsh, 1975).

In summary, while there is a need for more specific research to understand the mechanisms of spatial coding, the bulk of the evidence is more consistent with late than with early selection theories, and there is hardly any evidence suggesting that attentional shifting might precede and play a causal role in spatial-coding processes.

\section{Q3: Top-down cognitive control: Support of the cor- rect response and inhibitory control mechanisms}

The core of the third key question addresses the question whether one control mechanism involved in implementing the correct response is sufficient to select the correct response, or whether there is a need for an additional mechanism to inhibit the incorrect response, as suggested by the activation- suppression model (Ridderinkhof, 2002). ERP studies analysing activity in both motor cortices separately showed that motor cortex ipsilateral (but not contralateral) to the relevant correct response correlated with the degree of required inhibition (Burle, van den Wildenberg, Spieser, \& Ridderinkhof, 2016; Meckler et al., 2010; Servant, White, Montagnini \& Burle, 2016). This functional dissociation between ipsilateral and contralateral motor cortices activity was interpreted as evidence for the existence of one mechanism involved in select the correct response and another mechanism related to inhibition of the incorrect response. So, even if slow responses might allow spontaneous decay of the spatial code, fast responses (which are usually required when performing a Simon task) are argued to involve active inhibition of the irrelevant stimulus location or response in order to perform the task (Ridderinkhof, 2002).

The meta-analysis of fMRI studies carried out in the present study showed four clusters of activity associated with the Simon effect in frontal (middle cingulate cortex and medial frontal cortex; left middle frontal gyrus and left pre-central gyrus), parietal (right superior and inferior parietal lobes) and occipito-temporal (middle and inferior temporal gyri and middle occipital lobe) regions. Considering that activity to support the correct response did not change according to degree of conflict (Burle, van den Wildenberg, Spieser, \& Ridderinkhof 2016; Meckler et al., 2010; Servant, White, Montagnini \& Burle, 2016), the existence of higher neural activity in the incompatible condition than in the compatible condition may be related to inhibition of the response primed by the stimulus location.

One cluster of activity was observed in middle cingulate cortex and medial frontal cortex. These results are consistent with the view of dorsal ACC as a part of the brain network involved in conflict detection (Mansouri, Egner, \& Buckley, 2017). This cluster of activity would be consistent with increased theta activity in mid-frontal electrodes ( $\mathrm{Li}$ et al., 2015; van Driel, Swart, Egner, Ridderinkhof, \& Cohen, 2015; Vissers, Ridderinkhof, Cohen, \& Slagter, 2018; Wang, Li, Zheng, Wang, \& Liu, 2014) and increased ERP amplitudes in the incompatible compared to compatible trials. Specifically, fronto-central N200, which is thought to emerge from ACC (Folstein \& Van Petten, 2008), showed larger amplitude in the incompatible than in the compatible condition during Simon task (Carriero, Zalla, Budai, \& Battaglini, 2007; Chen \& Melara, 2009; Melara, Wang, Vu, \& Proctor, 2008) and other SRC tasks (Wendt, Heldmann, Münte, \& Kluwe, 2007). Another component related to ACC activity is MFN, which was involved in post-response conflict adaptation processes (Nieuwenhuis, Yeung, Holroyd, Schurger, \& Cohen, 2004; Van Veen \& Carter, 2002). Some studies showed larger MFN in highly demanding Simon tasks compared to easier versions of the Simon task (Masaki, Murphy, Desjardins, \& Segalowitz, 2012). 
The meta-analysis showed activity in left middle frontal gyrus and left pre-central gyrus associated with the Simon effect. This cluster may be related (at least partially) to dPM cortex, whose neurons modulate their firing rate according to specific S-R bindings (Abe \& Hanakawa, 2009; Beurze, Lange, Toni, \& Medendorp, 2007; Hoshi \& Tangji, 2000). According to Leuthold and Schröter (2006), N2cc represents an ERP correlate of a monitoring mechanism involved in preventing the spatial response. In fact, $\mathrm{N} 2 \mathrm{cc}$ is modulated by difficulty to prevent the spatial response (Cespón, GaldoÁlvarez, \& Díaz, 2012; Cespón, Galdo-Álvarez, \& Díaz, 2016; Leuthold \& Schröter, 2006). Depending on the specific parameters, TMS over left dPM is able to increase RT in both compatible and incompatible trials (Bardi, Schiff, Basso, \& Mapelli, 2015; Praamstra, Kleine \& Schnitzler, 1999). Altogether, these findings suggest that $\mathrm{dPM}$ is involved in activation of the spatially compatible response and selection of the correct response during the spatial conflict.

The right superior and inferior parietal lobes were also activated by the Simon effect, as revealed by the conducted meta-analysis. The posterior parietal cortex (PPC) is a region critically involved in early visuomotor integration (Stürmer \& Leuthold, 2003). PPC is essential for transforming the visual input into an appropriate motor output (Milner \& Goodale, 1995; Rushworth, Krams, \& Passingham, 2001), as confirmed by TMS studies in the Simon task (Schiff, Bardi, Basso, \& Mapelli, 2011; Stürmer, Redlich, Irlbacher, \& Brandt, 2007). PPC was related to spatial attention and reallocation of spatial attention (Molenberghs, Mesulam, Peeters, \& Vandenberghe, 2007; Yantis et al., 2002). It might require more resources in incompatible than compatible trials, although specific research would be needed to explore this hypothesis.

A fourth cluster of activation was found in the middle and inferior temporal gyri and middle occipital lobe. Previous studies did not report activity related to conflict and cognitive control in these regions. Nevertheless, this cluster of activity overlaps the neural sources of the N2pc, an ERP correlate of initiating the attentional shift (parietal source) and implementing the focus of attention to further analysing the stimulus features (occipito-temporal source) (Hopf et al., 2000; Lorenzo-López et al., 2011). In general, the N2pc is not altered by the Simon effect (Praasmtra \& Oostenveld, 2003; Van der Lubbe \& Verleger, 2002), although it was modulated in some versions of the Simon task containing a stimulus-stimulus overlap (Cespón, Galdo-Álvarez, \& Díaz, 2013c; Valle-Inclán, 1996). Therefore, we suggest that occipito-temporal activity related to processing of the stimulus features may increase in those trials where the stimulus itself contains conflicting spatial information (e.g., a left lateralised arrow pointing to the right). Crucially, further studies would be required to clarify the specific role of this region during S-R and S-S incompatibilities.
In short, neurophysiological studies using the Simon task provided evidence for neural activity related to support of the correct response and a set of areas (that is, ACC, dPM, and PPC) that interact, possible to orchestrate inhibitory control (and/or other cognitive functions sensitive to cognitive conflict). In light of this evidence, we should emphasise that the Simon task is a useful tool to investigate behavioural and neural correlates of conflict monitoring (Hommel, 2011a) and inhibition as well as inter-individual differences in these executive functions underlying factors related to cognitive reserve (Bialystok, 2017) or associated with brain pathology (Cespón, Galdo-Álvarez, \& Díaz, 2018; Zurrón et al., 2018). Future studies about functional connectivity may reveal how these areas interact to inhibit the incorrect response and/or implement the response based on the task instructions. Furthermore, upcoming studies may distinguish neural circuits specifically devoted to inhibit spatial information from neural areas activated across the different inhibitory control tasks and test the possibility that these functions relate to noninhibitory mechanisms sensitive to cognitive conflict, such as mechanisms subserving conflict monitoring, context integration, and learning.

\section{Q4: Concurrent activation of responses: Feedforward and mutual inhibition}

ERP research analysing the LRP component (De Jong, Liang, \& Lauber, 1994; Stürmer, Ouyang, Zhou, Boldt, \& Sommer, 2013; Valle-Inclán, 1996) and TMS studies investigating motor excitability (e.g., Stürmer, Siggelkow, Dengler, \& Leuthold, 2000; Van Campen, Keuken, van den Wildenberg, \& Ridderinkhof, 2014) suggest the existence of two concurrently active responses in the incompatible condition, which compete to achieve the critical response threshold. Thus, these studies provide evidence for the basic assumption of dual-route models: the concurrent activation of competing responses (De Jong, Liang, \& Lauber, 1994; Kornblum, Hasbroucq, \& Osman, 1990; Proctor \& Vu, 2006; Wascher, Schatz, Kuder, \& Verleger, 2001).

Some ERP studies stated that inhibition of incorrect responses relies on top-down cognitive control, which is implemented by prefrontal areas (Miller, 2000; Nyberg, 2018). Several ERP studies (e.g., Burle, Wery, Wildenberg, Spieser, \& Ridderinkhof, 2016) showed that activation and inhibition in both motor hemispheres were not anti-correlated, which was taken as evidence for the absence of reciprocal inhibition of competing responses and to suggest that inhibition is implemented by prefrontal areas in a feedforward manner (Burle, Wery, Wildenberg, Spieser, \& Ridderinkhof, 2016; Servant, White, Montagnini, \& Burle, 2016). In contrast, findings from a recent TMS study were taken as evidence for reciprocal inhibition (Treccani, Cona, Milanese, \& Umiltà, 2018). This study showed that increased excitability 
in the hemisphere involved in response execution was paralleled by decreased excitability in the contralateral area. Similar findings were obtained by using the flanker task (Verleger, Kuniecki, Möller, Fritzmannova, \& Siebner, 2009). Hence, top-down and reciprocal inhibition may act as synergic mechanisms involved in managing the competition between opposite responses, as suggested by some theoretical models of the Simon effect (e.g., Zorzi \& Umiltà, 1995). Even so, it is possible that reciprocal inhibition is not visible in the data under specific conditions. For example, when responses are made with two fingers of the same hand (Meynier, Burle, Possamai, Vidal, \& Hasbroucq, 2009), activation and inhibition processes would occur within the same hemisphere and the spatial resolution of EEG would be insufficient to distinguish the two processes.

Future studies should clarify whether there are specific experimental conditions or tasks associated with the hypothetical implementation of feedforward and/or reciprocal inhibition processes. Another open question is whether reciprocal inhibition of competing responses may simultaneously occur at various levels in the brain. Treccani, Cona, Milanese and Umiltà (2018) reported evidence for reciprocal inhibition between homologue motor cortices during response competition; however, it may occur at other levels in the brain. For instance, Verleger, Kuniecki, Möller, Fritzmannova, and Siebner (2009) suggested the existence of reciprocal inhibition processes between homologue dPM cortices during the performance of a flanker task.

\section{Q5: Temporal overlap of the activated responses and the size of the Simon effect}

The amount of neurophysiological research on cognitive control modulations according to the temporal overlap of the relevant and irrelevant dimensions is limited. Wascher (2005) showed reduced Simon effect as the time between stimulus localisation (as revealed by N2pc latency) and the manual response increased. Strack, Kaufmann, Kehrer, Brandt and Stürmer (2013) demonstrated that decreasing the temporal overlap between the relevant and the irrelevant stimulus dimensions gave rise to reduced fronto-central N200 amplitude and modulated activity in pre-frontal regions, as revealed by fMRI.

The available results are consistent with early research indicating that the interference size depends on the temporal overlap between irrelevant (i.e., spatial location) and relevant stimulus dimensions (De Jong, Liang, \& Lauber, 1994; Hommel, 1993). Additionally, these findings align with recent behavioural studies (Finkbeiner \& Heathcote, 2016; Ulrich, Schröter, Leuthold, \& Birngruber, 2015) stating that earlier (and not stronger) processing of the irrelevant than relevant dimension boosts the spatial response in incompatible trials and give rise to the Simon effect. Therefore, the Simon effect would be explained by a 'time-difference account' rather than a 'magnitude-difference account' between the relevant and the irrelevant features of the stimulus. Furthermore, the mentioned results support interpretations about different temporal dynamics of interference and cognitive control - as revealed by distributional analysis of reaction times (for a review, see Proctor, Miles, \& Baroni, 2011) - in terms of different speed of processing of the irrelevant dimension relative to the relevant dimension used in different SRC tasks.

\section{Q6: Sequential congruency effects: Conflict adaptation and feature integration}

Several studies aimed to clarify whether sequential effects were related to modulations of cognitive control and/or the updating of the previous S-R binding. ERP studies showed that parietal P300 - a correlate of speed for switching the S-R mapping and update working memory contents (AdroverRoig \& Barceló, 2010) - but not frontocentral N200 - a correlate of conflict monitoring and inhibitory control (Folstein \& Van Petten, 2008) - was modulated by sequential effects during the Simon task (Hoppe, Küper, \& Wascher, 2017). These observations provided evidence for feature integration as explanatory mechanism underlying sequential effects. Besides, another ERP study demonstrated that sequential effects were produced after trials without conflicting information (Chen \& Melara, 2009). Also, sequential effects were eliminated by experimental manipulations preventing integration of S-R features (Spapè, Band, \& Hommel, 2011) and increased conflict - along with higher midfrontal theta power - when probability of conflict was cued seems to speak against conflict adaptation premises (van Driel, Swart, Egner, Ridderinkhof, \& Cohen, 2015). In addition, congruency alternations (i.e., changes of the S-R binding in the ' $n$ ' with respect to ' $n-1$ ' trial) were associated with decreased theta power at parietal regions, a finding that was interpreted in line with the feature integration account (Gulbinaite, van Rijn, \& Cohen, 2014). Behavioural and electrophysiological (N200, P300, theta power) results from Cao, Cao, Yue, and Wang (2017) showing a reversed Simon effect by increasing the proportion of compatible trials have also implications for key question 6; specifically, reversed Simon effects due to decreasing the proportion of compatible trials cannot be explained by conflict adaptation. In this context, Li et al. (2015) found differences in RTs and $\mathrm{N} 200$ between $\mathrm{c}-\mathrm{C}$ and $\mathrm{i}-\mathrm{C}$ trials but, in contrast to the main prediction of conflict adaptation, they did not show differences between i-I and c-I trials. In general, results from EEG/ERP studies suggested that feature integration is the main mechanism underlying sequential congruency effects.

Whereas fMRI studies obtained patterns of results in line with the episodic retrieval account (i.e., feature integration) as mechanism related to sequential effects (Aisenberg, Sapir, Close, Henik, \& d'Avossa, 2018), other studies suggested that 
conflict adaptation can modulate the Simon effect on the basis of the previous trial (Egner, 2007; Horga et al., 2011). Nevertheless, these results might be explained by difficulty to entirely exclude feature integration in the experimental designs (Schmidt, Notebaert, \& Van Den Bussche, 2015). Structural (di Pellegrino, Ciaramelli, \& Làdavas, 2007) and functional MRI studies (e.g., Kerns, 2006) highlighted the importance of prefrontal regions in the generation of sequential effects. Accordingly, TMS applied over left DLPFC abolished sequential congruency effects (Stürmer et al., 2007). The reader may note that our meta-analysis of fMRI studies did not reveal DLPFC activity associated with the Simon effect even if it is a brain region classically related to cognitive control (MacDonald, Cohen, Stenger, \& Carter, 2000). Considering that $\mathrm{dPM}$ can be activated without recruiting DLPFC if the rules of a specific task are overlearned (Abe \& Hanakawa, 2009), which could happen during the Simon task performance, we suggest that DLPFC might be involved in working memory processes related to update of the S-R binding (which are crucial to produce the sequential effects), as proposed by Stürmer et al. (2007), whereas inhibition of the spatial response would rely on dPM activity.

In sum, the reviewed neurophysiological findings suggest that feature integration plays a major role in modulating the interference size on the basis of the previous trial. Also, conflict adaptation does not straightforwardly explain faster RTs in c-C than i-C sequences. The explanation for these results is that, after a conflicting trial, participants might use a more conservative criterion and for this reason RT is slow down in the next trial (Botvinick, Braver, Barch, Carter, \& Cohen, 2001; Li et al., 2015). This assumption renders conflict adaptation a non-parsimonious account because it needs to assume two different mechanisms to explain the pattern of data. Even so, it is not entirely clear whether and to what extent conflict adaptation and feature integration may co-exist as mechanisms underlying sequential effects during the Simon and other SRC tasks or whether they are two sides or aspects of the same mechanism.

\section{Conclusions}

The Simon effect is commonly attributed to stimulus-induced conflict related to response selection, that is, it is an effect resulting from the competition between a response activated through processing the stimulus location and a response activated based on the task instructions. Electrophysiological studies suggest that the process of encoding the stimulus location and the process of reacting to the stimulus overlap in time, which supports late attentional accounts such as the PMTA and the referential coding account. Moreover, integration of S-R features seems to play a crucial role in the activation of the spatial response. In order to perform the Simon task adequately, the cognitive system deploys top-down cognitive control mechanisms to support the correct response and a set of inhibitory mechanisms to prevent the stimulus-induced response, including top-down cognitive control exerted by prefrontal regions and (maybe) reciprocal inhibition of competing responses between homologue motor areas. The magnitudes of the conflict and underlying cognitive control correlates are modulated by the degree of temporal overlap between processing of the relevant dimension (e.g., the colour or the shape) and the irrelevant dimension (i.e., the spatial location) of the stimulus. The amount of conflict is also affected by the previous trial, a phenomenon labelled as sequential effects. Neurophysiological research suggests that, according to the episodic retrieval account, the sequential congruency effects strongly depend on the integration of S-R features.

In light of the reviewed research, we emphasise the utility of the Simon task to investigate correlates of attentional, executive (including inhibition, monitoring, and switching), and motor processes in healthy and clinical populations. In general, neurophysiological tools were successfully used to shed light on the raised theoretical questions; however, a number of issues remain still open. For example, the experimental conditions and timing of formation of the spatial code should be clarified, and whether some proposed but controversial mechanisms, such as mutual inhibition, of competing responses and conflict adaptation underlying sequential effects may occur in some specific experimental conditions but not in others. The combination of TMS with fMRI and EEG/ERP techniques in the context of appropriate experimental designs opens a new window to obtain valuable information about those unresolved research questions.

Acknowledgements This research was supported by the Basque Government through the BERC 2018-2021 program and by the Spanish State Research Agency through BCBL Severo Ochoa excellence accreditation SEV-2015-0490 This study was also funded by Juan de la Cierva-Incorporación (Spanish government; JC) and European Commission (Marie Skłodowska-Curie actions 838536 BILINGUALPLAS; JC), by post-doctoral funding of the University of Bremen (DG; ZF 11/876/08), and by an ERC Advanced Grant (BH: ERC-2015-AdG-694722).

\section{Compliance with ethical standards}

Conflicts of interest The authors declare that there are no actual or potential conflicts of interest.

Open practices statement Data and/or materials of the performed metaanalysis are available in the Online Supplementary Materials. Experiments or sections of this study were not preregistered.

\section{References}

Abe, M., \& Hanakawa, T. (2009). Functional coupling underlying motor and cognitive functions of the dorsal premotor cortex. Behavioural 
Brain Research, 198(1), 13-23. https://doi.org/10.1016/j.bbr.2008. 10.046 .

Abrahamse, E., Braem, S., Notebaert, W., \& Verguts, T. (2016). Grounding cognitive control in associative learning. Psychological Bulletin, 142(7), 693-728. https://doi.org/10.1037/bul0000047.

Abrahamse, E., \& Van der Lubbe, R. (2008). Endogenous orienting modulates the Simon effect: critical factors in experimental design. Psychological Research, 72(3), 261-272. https://doi.org/10.1007/ s00426-007-0110-x.

Acosta, E, \& Simon, J. R. (1976). The effect of irrelevant information on the stages of processing. Journal of Motor Behavior, 8(3), 181-187. https://doi.org/10.1080/00222895.1976.10735070.

Adrover-Roig, D., \& Barceló, F. (2010). Individual differences in aging and cognitive control modulate the neural indexes of context updating and maintenance during task switching. Cortex, 46(4), 434-450. https://doi.org/10.1016/j.cortex.2009.09.012.

Aisenberg, D., Sapir, A., Close, A., Henik, A., \& d'Avossa, G. (2018). Right anterior cerebellum BOLD responses reflect age related changes in Simon task sequential effects. Neuropsychologia, 109, 155-164. https://doi.org/10.1016/j.neuropsychologia.2017.12.012.

Andersen, R. A., \& Buneo, C. A. (2002). Intentional maps in posterior parietal cortex. Annual Review of Neuroscience, 25, 189-220. https://doi.org/10.1146/annurev.neuro.25.112701.142922.

Andreou, C., Frielinghaus, H., Rauh, J., Mußmann, M., Vauth, S., Braun, P., Leicht, G., Mulert, C. (2017). Theta and high-beta networks for feedback processing: a simultaneous EEG-fMRI study in healthy male subjects. Translational Psychiatry, 7(1), e1016-e1016. https:// doi.org/10.1038/tp.2016.287.

Atalay, N. B., \& Misirlisoy, M. (2012). Can contingency learning alone account for item-specific control? Evidence from within- and between-language ISPC effects. Journal of Experimental Psychology: Learning, Memory, and Cognition, 38(6), 1578-1590. https://doi.org/10.1037/a0028458.

Bardi, L., Kanai, R., Mapelli, D., \& Walsh, V. (2012). TMS of the FEF interferes with spatial conflict. Journal of Cognitive Neuroscience, 24, 1305-1313. https://doi.org/10.1162/jocn_a_00223.

Bardi, L., Schiff, S., Basso, D., \& Mapelli, D. (2015). A transcranial magnetic stimulation study on response activation and selection in spatial conflict. European Journal of Neuroscience, 41, 487-491. https://doi.org/10.1111/ejn.12803.

Barker, A. T., Jalinous, R., \& Freeston, I. L. (1985). Non-invasive magnetic stimulation of human motor cortex. Lancet, 1(8437), 11061107. https://doi.org/10.1016/S0140-6736(85)92413-4.

Bartholow, B.D., Pearson, M.A., Dickter, C.L., Sher, K.J., Fabiani, M., \& Gratton, G. (2005). Strategic control and medial frontal negativity: beyond errors and response conflict. Psychophysiology, 42(1), 33-42.

Bernat, E. M., Nelson, L. D., \& Baskin-Sommers, A. R. (2015). Timefrequency theta and delta measures index separable components of feedback processing in a gambling task. Psychophysiology, 52(5), 626-637. https://doi.org/10.1111/psyp.12390.

Beurze, S. M., Lange, F. P., Toni, I., \& Medendorp, W. P. (2007). Integration of target and effector information in the human brain during reach planning. Journal of Neurophysiology, 97, 188-199. https://doi.org/10.1152/jn.00456.2006.

Bialystok, E. (2017). The bilingual adaptation: how minds accommodate experience. Psychological Bulletin, 143(3), 233-262. https://doi.org/ 10.1037/bul0000099.

Böckler, A., Alpay, G., \& Stürmer, B. (2011). Accessory stimuli affect the emergence of conflict, not conflict control: A Simon-task ERP study. Experimental Psychology, 58, 102-109. https://doi.org/10. 1027/1618-3169/a000073.

Bogacz, R. (2007). Optimal decision-making theories: linking neurobiology with behaviour. Trends in Cognitive Sciences, 11, 118-125. https://doi.org/10.1016/j.tics.2006.12.006.

Bogacz, R., Brown, E., Moehlis, J., Homes, P., \& Cohen, J. D. (2006). The physics of optimal decision making: A formal analysis of models of performance in two-alternative forced-choice tasks. Psychological Review, 113(4), 700-765. https://doi.org/10.1037/ 0033-295X.113.4.700.

Botvinick, M. M., Braver, T. S., Barch, D. M., Carter, C. S., \& Cohen, J. D. (2001). Conflict monitoring and cognitive control. Psychological Review, 108(3), 624-652. https://doi.org/10.1037/0033-295X.108. 3.624 .

Burke, M. J., Fried, P. J., \& Pascual-Leone, A. (2019). Transcranial magnetic stimulation: Neurophysiological and clinical applications. Handbook of Clinical Neurology, 163, 73-92. https://doi.org/10. 1016/B978-0-12-804281-6.00005-7.

Burle, B., Spieser, L., Roger, C., Casini, L., Hasbroucq, T., \& Vidal, F. (2015). Spatial and temportal resolutions of EEG: Is it really black and white? A scalp current density view. International Journal of Psychophysiology, 97, 210-220. https://doi.org/10.1016/j.ijpsycho. 2015.05.004.

Burle, B., van den Wildenberg W. P., Spieser L., \& Ridderinkhof K. R. (2016). Preventing (impulsive) errors: Electrophysiological evidence for online inhibitory control over incorrect responses. Psychophysiology, 53(7), 1008-1019. https://doi.org/10.1111/psyp. 12647.

Bush, G., Luu, P., \& Posner, M. I. (2000). Cognitive and emotional influences in anterior cingulate cortex. Trends in Cognitive Sciences, 4(6), 215-222. https://doi.org/10.1016/S1364-6613(00) 01483-2.

Cao, Y., Cao, X., Yue, Z., \& Wang, L. (2017). Temporal and spectral dynamics underlying cognitive control modulated by task-irrelevant stimulus-response learning. Cognitive, Affective, \& Behavioral Neuroscience, 17(1), 158-173. https://doi.org/10.3758/s13415-0160469-5.

Carriero, L., Zalla, T., Budai, R., \& Battaglini, P.P. (2007). Inhibition of wrong responses and conflict resolution: an electroencephalogram study. Neuroreport, 18(8), 793-796. https://doi.org/10.1097/WNR. 0b013e3280c1e330.

Cavanagh, J. F., \& Frank, M. J. (2014). Frontal theta as a mechanism for cognitive control. Trends in Cognitive Sciences, 18(8), 414-421. https://doi.org/10.1016/j.tics.2014.04.012.

Cespón, J., Galdo-Álvarez, S., \& Díaz, F. (2012). The Simon effect modulates N2cc and LRP but not the N2pc component. International Journal of Psychophysiology, 84(2), 120-129. https://doi.org/10. 1016/j.ijpsycho.2012.01.019.

Cespón, J., Galdo-Álvarez, S., \& Díaz, F. (2013a). Similarities and differences between interference from stimulus position and from direction of an arrow: Behavioral and event-related potential measures. International Journal of Psychophysiology, 90(2), 180-189. https://doi.org/10.1016/j.ijpsycho.2013.07.002.

Cespón, J., Galdo-Álvarez, S., \& Díaz, F. (2013b). Age-related changes in ERP correlates of visuospatial and motor processes. Psychophysiology, 50(8), 743-757. https://doi.org/10.1111/psyp. 12063.

Cespón J, Galdo-Álvarez S, \& Díaz F. (2013c). N2pc is modulated by stimulus-stimulus, but not by stimulus-response incompatibilities. Biological Psychology, 93(1), 75-80. https://doi.org/10.1016/j. biopsycho.2013.01.010.

Cespón, J., Galdo-Álvarez, S., \& Díaz F. (2016). Cognitive control activity is modulated by the magnitude of interference and preactivation of monitoring mechanisms. Scientific Reports, 6:39595. https://doi.org/10.1038/srep39595.

Cespón, J., Galdo-Álvarez, S., \& Díaz, F. (2018). Event-related potentials reveal altered executive control activity in healthy elderly with subjective memory complaints. Frontiers in Human Neuroscience, 12: 445. https://doi.org/10.3389/fnhum.2018.00445r.

Chen, C., Muggleton, N. G., Tzeng, O. J. L., Hung, D. L., \& Juan, C. (2009). Control of prepotent responses by the superior medial frontal cortex. Neuroimage, 44(2), 537-545. https://doi.org/10.1016/j. neuroimage.2008.09.005. 
Chen, S., \& Melara, R. D. (2009). Sequential effects in the Simon task: conflict adaptation or feature integration? Brain Research, 1297, 89100. https://doi.org/10.1016/j.brainres.2009.08.003.

Cieslik, E. C., Mueller, V. I., Eickhoff, C. R., Langner, R., \& Eickhoff, S. B. (2015). Three key regions for supervisory attentional control: Evidence from neuroimaging meta-analyses. Neuroscience \& Biobehavioral Reviews, 48, 22-34. https://doi.org/10.1016/j. neubiorev.2014.11.003.

Cohen, M. X. (2014). A neural microcircuit for cognitive conflict detection and signalling. Trends in Neurosciences, 37(9), 480-490. https://doi.org/10.1016/j.tins.2014.06.004.

Cohen, M. X., \& Cavanagh, J. F. (2011). Single-trial regression elucidates the role of prefrontal theta oscillations in response conflict. Frontiers in Psychology, 2, 30. https://doi.org/10.3389/fpsyg.2011. 00030.

Cohen, M. X., \& Donner, T. H. (2013). Midfrontal conflict-related thetaband power reflects neural oscillations that predict behavior. Journal of Neurophysiology, 110(12), 2752-2763. https://doi.org/10.1152/ jn.00479.2013.

Cohen, M. X., \& Ridderinkhof, K. R. (2013). EEG source reconstruction reveals frontal-parietal dynamics of spatial conflict processing. PLoS One, 8(2), e57293. https://doi.org/10.1371/journal.pone. 0057293.

Coles, M. G. H. (1989). Modern mind-brain reading: Psychophysiology, physiology, and cognition. Psychophysiology, 26, 251-269. https:// doi.org/10.1111/j.1469-8986.1989.tb01916.x

Cona, G., Treccani, B., \& Umiltà, C. A. (2016). Is cognitive control automatic? New insights from transcranial magnetic stimulation. Psychonomic Bulletin and Review, 23(5), 1624-1630. https://doi. org/10.3758/s13423-016-1023-8.

Craft, J. L., \& Simon, J. R. (1970). Processing symbolic information from a visual display: Interference from an irrelevant directional cue. Journal of Experimental Psychology, 83(3), 415-420. https://doi. org/10.1037/h0028843.

De Houwer, J. (2003). On the role of stimulus-response and stimulusstimulus compatibility in the Stroop effect. Memory \& Cognition, 31, 353-359. http://hdl.handle.net/1854/LU-167328.

De Jong, R., Liang, C.C., \& Lauber, E. (1994). Conditional and unconditional automaticity: a dual-process model of effects of spatial stimulus-response correspondence. Journal of Experimental Psychology: Human Perception and Performance, 20(4), 731-750. https://doi.org/10.1037/0096-1523.20.4.731.

De Jong, R., Wierda, M., Mulder, G., \& Mulder, L. J. M. (1988). Use of partial information in response processing. Journal of Experimental Psychology: Human Perception and Perfromance, 14, 682-692. https://doi.org/10.1037/0096-1523.14.4.682.

Desender, K., \& Van den Bussche, E. (2012). Is consciousness necessary for conflict adaptation? A state of the art. Frontiers in Human Neuroscience, 6, 3. https://doi.org/10.3389/fnhum.2012.00003.

di Pellegrino, G., Ciaramelli, E., \& Làdavas, E. (2007). The regulation of cognitive control following rostral anterior cingulate cortex lesion in humans. Journal of Cognitive Neuroscience, 19, 275-286. https:// doi.org/10.1162/jocn.2007.19.2.275

Dignath, D., Johannsen, L., Hommel, B., \& Kiesel, A. (2019). Contextual control of conflict: Reconciling cognitive-control and episodicretrieval accounts of sequential conflict modulation. Journal of Experimental Psychology: Human Perception and Performance, 45, 1265-1270.

Dittrich, K., Kellen, D., \& Stahl, C. (2014). Analyzing distributional properties of interference effects across modalities: chances and challenges. Psychological Research, 78, 387-399. https://doi.org/ 10.1007/s00426-014-0551-y.

Donchin, E., \& Coles, M. G. H. (1988). Is the P300 component a manifestation of context updating? Behavioral and Brain Sciences, 11, 357-374. https://doi.org/10.1017/S0140525X00058027.
Dreisbach, G., \& Fischer, R. (2015). Conflicts as aversive signals for conflict adaptation. Current Directions in Psychological Science, 24(4), 255-260. https://doi.org/10.1177/0963721415569569.

Duncan, J. (2010). The multiple-demand (MD) system of the primate brain: mental programs for intelligent behaviour. Trends in Cognitive Sciences, 14(4), 172-179. https://doi.org/10.1016/j.tics. 2010.01.004.

Duthoo, W., Abrahamse, E. L., Braem, S., Boehler, C. N., \& Notebaert, W. (2014). The congruency sequence effect 3.0: A critical test of conflict adaptation. PLoS One, 9(10), e110462. https://doi.org/10. 1371/journal.pone.0110462.

Egner, T. (2007). Congruency sequence effects and cognitive control. Cognitive, Affective, \& Behavioral Neuroscience, 7, 380-390. https://doi.org/10.3758/CABN.7.4.380.

Egner T. (2014). Creatures of habit (and control): a multi-level learning perspective on the modulation of congruency effects. Frontiers in Psychology, 5(1247). https://doi.org/10.3389/fpsyg.2014.01247.

Egner, T., Delano, M., \& Hirsch, J. (2007). Separate conflict-specific cognitive control mechanisms in the human brain. Neuroimage, 35, 940-948. https://doi.org/10.1016/j.neuroimage.2006.11.061.

Egner, T., \& Hirsch, J. (2005). Cognitive control mechanisms resolve conflict through cortical amplification of task-relevant information. Nature Neuroscience, 8, 1784-1790. https://doi.org/10.1038/ nn1594.

Eickhoff, S. B., Bzdok, D., Laird, A. R., Kurth, F., \& Fox, P. T. (2012). Activation likelihood estimation meta-analysis revisited. Neuroimage, 59(3), 2349-2361. https://doi.org/10.1016/j. neuroimage.2011.09.017.

Eickhoff, S. B., Laird, A. R., Grefkes, C., Wang, L. E., Zilles, K., \& Fox, P. T. (2009). Coordinate-based activation likelihood estimation meta-analysis of neuroimaging data: A random-effects approach based on empirical estimates of spatial uncertainty. Human Brain Mapping, 30(9), 2907-2926. https://doi.org/10.1002/hbm.20718.

Eimer, M. (1996). The N2pc component as an indicator of attentional selectivity. Electroencephalography and Clinical Neurophysiology, 99(3), 225-234. https://doi.org/10.1016/00134694(96)95711-9.

Eimer, M., Forster, B., Van Velzen, J., \& Prabhu, G. (2005). Covert manual response preparation triggers attentional shifts: ERP evidence for the premotor theory of attention. Neuropsychologia, 43(6), 957-966. https://doi.org/10.1016/j.neuropsychologia.2004. 08.011 .

Eimer, M., Van Velzen, J., Gherri, E., \& Press, C. (2007). ERP correlates of shared control mechanisms involved in saccade preparation and in covert attention. Brain Reseach, 1135(1), 154-166. https://doi. org/10.1016/j.brainres.2006.12.007.

Eriksen, B. A., \& Eriksen, C. W. (1974). Effects of noise letters upon identification of a target letter in a non-search task. Perception \& Psychophysics, 16, 143-149. https://doi.org/10.3758/BF03203267.

Finkbeiner, M., \& Heathcote, A. (2016). Distinguishing the time- and magnitude-difference accounts of the Simon effect: Evidence from the reach-to-touch paradigm. Attention, Perception \& Psychophysics, 78(3), 848-867. https://doi.org/10.3758/s13414015-1044-9.

Folstein, J. R., \& Van Petten, C. (2008). Influence of cognitive control and mismatch on the N2 component of the ERP: A review. Psychophysiology, 45, 152-170. https://doi.org/10.1111/j.14698986.2007.00602.x.

Forstmann, B. U., Jahfari, S., Scholte, H. S., Wolfensteller, U., van den Wildenberg, Wery P. M., \& Ridderinkhof, K. R. (2008a). Function and structure of the right inferior frontal cortex predict individual differences in response inhibition: A model-based approach. Journal of Neuroscience, 28(39), 9790-9796. https://doi.org/10. 1523/JNEUROSCI.1465-08.2008.

Forstmann, B. U., van den Wildenberg, Wery P. M., \& Ridderinkhof, K. R. (2008b). Neural mechanisms, temporal dynamics, and individual 
differences in interference control. Journal of Cognitive Neuroscience, 20(10), 1854-1865. https://doi.org/10.1162/jocn. 2008.20122 .

Fournier, L. R., Scheffers, M. K., Coles, M. G. H., Adamson, A., \& Villa Abad, E. (1997). The dimensionality of the flanker compatibility effect: A psychophysiological analyses. Psychological Research, 60, 144-155. https://doi.org/10.1007/BF00419762.

Fröber, K., Stürmer, B., Frömer, R., \& Dreisbach, G. (2017). The role of affective evaluation in conflict adaptation: An LRP study. Brain and Cognition, 116, 9-16. https://doi.org/10.1016/j.bandc.2017.05.003.

Frühholz, S., Godde, B., Finke, M. \& Herrmann, M. (2011). Spatiotemporal brain dynamics in a combined stimulus-stimulus and stimulus-response conflict task. NeuroImage, 54(1), 622-634. https://doi.org/10.1016/j.neuroimage.2010.07.071.

Georgiou-Karistianis, N., Akhlaghi, H., Corben, L. A., Delatycki, M. B., Storey, E., Bradshaw, J. L., \& Egan, G. F. (2012). Decreased functional brain activation in Friedreich ataxia using the Simon effect task. Brain and Cognition, 79(3), 200. https://doi.org/10.1016/j. bandc.2012.02.011.

Grahn, J. A., Parkinson, J. A., \& Owen, A. M. (2008). The cognitive functions of the caudate nucleus. Progress in Neurobiology, 86(3), 141-155. https://doi.org/10.1016/j.pneurobio.2008.09.004.

Grandjean, J., D'Ostilio, K., Fias, W., Phillips, C., Balteau, E., Degueldre, C., Luxen, A., Maquet, P., Salmon, E., \& Collette, F. (2013). Exploration of the mechanisms underlying the ISPC effect: Evidence from behavioral and neuroimaging data. Neuropsychologia, 51(6), 1040-1049. https://doi.org/10.1016/j. neuropsychologia.2013.02.015.

Gratton, G., Coles, M. G., \& Donchin, E. (1992). Optimizing the use of information: strategic control of activation of responses. Journal of Experimental Psychology: General, 121, 480-506. https://doi.org/ 10.1037/0096-3445.121.4.480.

Gratton, G., Coles, M. G. H., Sirevaag, E. J., Eriksen, C. W., \& Donchin, E. (1988). Pre-and poststimulus activation of response channels: a psychophysiological analysis. Journal of Experimental Psychology: Human Perception and Performance, 14 (3), 331-344. https://doi. org/10.1037/0096-1523.14.3.331.

Grosbras, M. H., \& Paus, T. (2002). Transcranial magnetic stimulation of the human frontal eye field: effects on visual perception and attention. Journal of Cognitive Neuroscience, 14, 1109-1120. https://doi. org/10.1162/089892902320474553.

Gulbinaite, R., van Rijn, H., \& Cohen, M. X. (2014). Fronto-parietal network oscillations reveal relationship between working memory capacity and cognitive control. Frontiers in Human Neuroscience, 8, 761. https://doi.org/10.3389/fnhum.2014.00761

Gundel, A., \& Wilson, G. F. (1992). Topographical changes in the ongoing EEG related to the difficulty of mental tasks. Brain Topography, 5(1), 17-25. https://doi.org/10.1007/BF01129966.

Hanslmayr, S., Pastötter, B., Bäuml, K. H., Gruber, S., Wimber, M., \& Klimesch, W. (2008). The electrophysiological dynamics of interference during the Stroop task. Journal of Cognitive Neuroscience, 20(2), 215-225. https://doi.org/10.1162/jocn.2008.20020.

Hasbroucq, T., \& Guiard, Y. (1991). Stimulus-response compatibility and the Simon effect: Toward a conceptual clarification. Journal of Experimental Psychology: Human Perception and Performance, 17, 2465-2266. https://doi.org/10.1037/0096-1523.17.1.246.

Hasbroucq, T., \& Guiard, Y. (1992). The effects of intensity and irrelevant location of a tactile stimulation in a choice reaction time task. Neuropsychologia, 30(1), 91-94.

Hedge, A., \& Marsh, N. W. (1975). The effect of irrelevant spatial correspondences on two-choice response-time. Acta Psychologica, 39(6), 427-439. https://doi.org/10.1016/0001-6918(75)90041-4.

Herz, D. M., Chistensen, M. S., Bruggemann, N., Hulme, O. J., Ridderinkhof, K. R., Madsen, K. H., \& Siebner, H. R. (2014). Motivational tuning of fronto-subthalamic connectivity facilitates control of action impulses. Journal of Neuroscience, 34(9), 32103217. https://doi.org/10.1523/JNEUROSCI.4081-13.2014.

Hommel, B. (1993). The role of attention for the Simon effect. Psychological Bulletin, 55, 208-222. https://doi.org/10.1007/ BF00419608.

Hommel, B. (1994). Spontaneous decay of response-code activation. Psychological Research, 56(4), 261-268.

Hommel, B. (1995). Stimulus-response compatibility and the Simon effect: Toward an empirical clarification. Journal of Experimental Psychology: Human Perception and Performance, 21, 764-775. https://doi.org/10.1037/0096-1523.17.1.246.

Hommel, B. (2004). Event files: feature binding in and across perception and action. Trends in Cognitive Sciences, 8(11), 494-500. https:// doi.org/10.1016/j.tics.2004.08.007.

Hommel, B. (2011a). The Simon effect as tool and heuristic. Acta Psychologica, 136, 189-202. https://doi.org/10.1016/j.actpsy.2010. 04.011.

Hommel, B. (2011b). Attention and spatial stimulus coding in the Simon task: a rejoinder to van der Lubbe and Abrahamse (2010). Acta Psychologica, 136(2), 265-268. https://doi.org/10.1016/j.actpsy. 2010.10.002

Hommel, B. \& Lippa Y. (1995). S-R compatibility effects due to contextdependent spatial stimulus coding. Psychonomic Bulletin \& Review, 2(3), 370-374. https://doi.org/10.3758/BF03210974.

Hommel, B., Müsseler, J., Aschersleben, G., \& Prinz, W. (2001). The Theory of Event Coding (TEC): a framework for perception and action planning. Behavioral and Brain Sciences, 24(5), 849-878. https://doi.org/10.1017/S0140525X01000103.

Hommel, B., Proctor, R. W. \& Vu, K. P. L. (2004). A feature integration account of sequential effects in the Simon task. Psychological Research, 68, 1-17. https://doi.org/10.1007/s00426-003-0132-y.

Hopf, J. M., Luck, S. J., Girelli, M., Hagner, T., Mangun, G. R., Scheich, H., \& Heinze, H. J. (2000). Neural sources of focused attention in visual search. Cerebral Cortex, 10, 1233-1241. https://doi.org/10. 1093/cercor/10.12.1233.

Hoppe, K., Küper, K., \& Wascher, E. (2017). Sequential modulations in a combined horizontal and vertical Simon task: is there ERP evidence for feature integration effects? Frontiers in Psychology, 8:1094. https://doi.org/10.3389/fpsyg.2017.01094.

Horga, G., Maia, T. V., Wang, P., Wang, Z., Marsh, R., \& Peterson, B. S. (2011). Adaptation to conflict via context-driven anticipatory signals in the dorsomedial prefrontal cortex. Journal of Neuroscience, 31, 16208-16216. https://doi.org/10.1523/JNEUROSCI.2783-11. 2011.

Hoshi, E., \& Tangji, J. (2000). Integration of target and body-part information in the premotor cortex when planning action. Nature, 408, 466-470. https://doi.org/10.1038/35044075.

Iani, C., Ricci, F., Baroni, G., \& Rubichi, S. (2009). Attention control and susceptibility to hypnosis. Consciousness and Cognition: An International Journal, 18(4), 856-863. https://doi.org/10.1016/j. concog.2009.07.002.

Jacobs, J., Hwang, G., Curran, T., \& Kahana, M. J. (2006). EEG oscillations and recognition memory: theta correlates of memory retrieval and decision making. Neuroimage, 32(2), 978-987. https://doi.org/ 10.1016/j.neuroimage.2006.02.018.

Jahfari, S., Waldorp, L., van den Wildenberg, W. P. M., Scholte, H. S., Ridderinkhof, K. R., \& Forstmann, B. U. (2011). Effective connectivity reveals important roles for both the hyperdirect (frontosubthalamic) and indirect (fronto-striatal-pallidal) fronto-basal ganglia pathways during response inhibition. Journal of Neuroscience, 31(18), 6891-6899. https://doi.org/10.1523/JNEUROSCI.5253-10. 2011.

Jiang, J., \& Egner, T. (2014). Using neural pattern classifiers to quantify the modularity of conflict-control mechanisms in the human brain. Cerebral Cortex, 24(7), 1793-1805. https://doi.org/10.1093/cercor/ bht029. 
Johansen-Berg, H., Rushworth, M. F. S., Bogdanovic, M. D., Kischka, U., Wimalaratna, S., \& Matthews, P. M. (2002). The role of ipsilateral premotor cortex in hand movement after stroke. Proceedings of the National Academy of Sciences of the United States of America, 99(22), 14518-14523. https://doi.org/10.1073/pnas.222536799.

Karayanidis, F., Whitson, L.R., Heathcote, A., \& Michie, P.T. (2011). Variability in proactive and reactive cognitive control processes across the adult lifespan. Frontiers in Psychology, 2, 318. https:// doi.org/10.3389/fpsyg.2011.00318.

Kayser, J., \& Tenke, C. E. (2015). On the benefits of using surface Laplacian (current source density) methodology in electrophysiology. International Journal of Psychophysiology, 97(3), 171-173. https://doi.org/10.1016/j.ijpsycho.2015.06.001.

Kerns J. G. (2006). Anterior cingulate and prefrontal cortex activity in an FMRI study of trial-to-trial adjustments on the Simon task. Neuroimage, 33, 399-405. https://doi.org/10.1016/j.neuroimage. 2006.06.012.

Kerns, J. G., Cohen, J. D., MacDonald, A. W., Cho, R. Y., Stenger, V. A., \& Carter, C. S. (2004). Anterior cingulate conflict monitoring and adjustments in control. Science, 303, 1023-1026. https://doi.org/10. 1126/science. 1089910.

Kieffaber, P. D., \& Hetrick, W. P. (2005). Event-related potential correlates of task switching and switching costs. Psychophysiology, 42, 56-71. https://doi.org/10.1111/j.1469-8986.2005.00262.x.

Kim, C., Johnson, N. F., \& Gold, B. T. (2012). Common and distinct neural mechanisms of attentional switching and response conflict. Brain Research, 1469, 92-102. https://doi.org/10.1016/j.brainres. 2012.06.013.

Kim, S., \& Cho, Y. S. (2014). Congruency sequence effect without feature integration and contingency learning. Acta Psychologica, 149, 60-68. https://doi.org/10.1016/j.actpsy.2014.03.004.

Klein, R. M., \& Ivanoff, J. (2011). The components of visual attention and the ubiquitous Simon effect. Acta Psychologica, 136(2), 225234. https://doi.org/10.1016/j.actpsy.2010.08.003.

Koch, G., \& Rothwell, J. C. (2009). TMS investigations into the taskdependent functional interplay between human posterior parietal and motor cortex. Behavioural Brain Research 202(2), 147-152. https://doi.org/10.1016/j.bbr.2009.03.023.

Kornblum, S., Hasbroucq, T., \& Osman, A. (1990). Dimensional overlap: cognitive basis for stimulus-response compatibility: A model and taxonomy. Psychological Review, 97(2), 253-270. https://doi.org/ 10.1037/0033-295X.97.2.253.

Kornhuber, H. H., \& Deecke, L. (1965). Hirnpotentialänderungen bei Willkürbewegungen und passiven Bewegungen des Menschen: Bereitschaftspotential und reafferente Potentiale. Pflügers Archiv für die gesamte Psychologie, 284, 1-17. https://doi.org/10.1007/ BF00412364.

Korsch, M., Frühholz, S., \& Herrmann, M. (2014). Ageing differentially affects neural processing of different conflict types-an fMRI study. Frontiers in Aging Neuroscience, 6:57. https://doi.org/10.3389/ fnagi.2014.00057.

Kühn, S., Keizer, A., Rombouts, S. A. R. B., \& Hommel, B. (2011). The functional and neural mechanism of action preparation: roles of EBA and FFA in voluntary action control. Journal of Cognitive Neuroscience, 23, 214-220. https://doi.org/10.1162/jocn.2010. 21418.

Kutas, M., McCarthy, G., \& Donchin, E. (1977). Augmenting mental chronometry: The P300 as a measure of stimulus evaluation time. Science, 197, 792-795. https://doi.org/10.1126/science.887923.

Lamberts, K., Tavernier, G., \& d'Ydewalle, G. (1992). Effects of multiple reference points in spatial stimulus-response compatibility. Acta Psychologica 79(2), 115-130. https://doi.org/10.1016/00016918(92)90028-C.

Lamers, M. J., \& Roelofs, A. (2011). Attentional control adjustments in Eriksen and Stroop task performance can be independent of response conflict. Quarterly Journal of Experimental Psychology, 64(6), 1056-1081. https://doi.org/10.1080/17470218.2010.523792.

Lancaster, J. L., Woldorff, M. G., Parsons, L. M., Liotti, M., Freitas, C. S., Rainey, L., ... Fox, P.T. (2000). Automated talairach atlas labels for functional brain mapping. Human Brain Mapping, 10(3), 120131. https://doi.org/10.1002/1097-0193(200007)10:33.0.CO;2-8.

Larson, M. J., Clayson, P. E., \& Clawson, A. (2014). Making sense of all the conflict: A theoretical review and critique of conflict-related ERPs. International Journal of Psychophysiology, 93(3), 283-297. https://doi.org/10.1016/j.ijpsycho.2014.06.007.

Leuthold, H. (2011). The Simon effect in cognitive electrophysiology: a short review. Acta Psychologica, 136, 203-211. https://doi.org/10. 1016/j.actpsy.2010.08.001.

Leuthold, H., \& Schröter, H. (2006). Electrophysiological evidence for response priming and conflict regulation in the auditory Simon task. Brain Research, 1097, 167-180. https://doi.org/10.1016/j.brainres. 2006.04.055.

Leuthold, H., \& Sommer, W. (1999). ERP correlates of error processing in spatial S-R compatibility tasks. Clinical Neurophysiology, 110(2), 342-357. https://doi.org/10.1016/S1388-2457(98)00058-3.

Li, Q., Wang, K., Nan, W., Zheng, Y., Wu, H., Wang, H., \& Liu, X. (2015). Electrophysiological dynamics reveal distinct processing of stimulus-stimulus and stimulus-response conflicts. Psychophysiology, 52(4), 562-571. https://doi.org/10.1111/psyp. 12382.

Li, H., Xia, T., \& Wang, L. (2015). Neural correlates of the reverse Simon effect in the Hedge and Marsh task. Neuropsychologia, 75, 119-131.

Li, Q., Yang, G., Li, Z., Qi, Y., Cole, M. W., \& Liu, X. (2017). Conflict detection and resolution rely on a combination of common and distinct cognitive control networks. Neuroscience \& Biobehavioral Reviews, 83, 123-131. https://doi.org/10.1016/j.neubiorev.2017.09. 032.

Lorenzo-López, L., Gutiérrez, R., Moratti, S., Maestú, F., Cadaveira, F., \& Amenedo, E. (2011). Age-related occipito-temporal hypoactivation during visual search: relationships between $\mathrm{mN} 2 \mathrm{pc}$ sources and performance. Neuropsychologia, 49(5), 858-865. https://doi.org/10.1016/j.neuropsychologia.2011.01.015.

Lu, C., \& Proctor, R. W. (1995). The influence of irrelevant location information on performance: A review of the Simon and spatial Stroop effects. Psychonomic Bulletin \& Review, 2, 174-207. https://doi.org/10.3758/BF03210959

Van der Lubbe, R. H. J., \& Verleger, R. (2002). Aging and the Simon task. Psychophysiology, 39(01), 100-110. https://doi.org/10.1111/ 1469-8986.3910100.

Luck, S. J., Girelli, M., McDermott, M. T., \& Ford, M. A. (1997). Bridging the gap between monkey neurophysiology and human perception: an ambiguity resolution theory of visual selective attention. Cognitive Psychology, 33, 64-87. https://doi.org/10.1006/ cogp.1997.0660.

Luck, S. J., \& Hillyard, S. A. (1994). Spatial filtering during visual search: Evidence from human electrophysiology. Journal of Experimental Psychology: Human Perception and Performance, 20, 1000-1014. https://doi.org/10.1037/0096-1523.20.5.1000.

MacDonald, A. W., Cohen, J. D., Stenger, V. A., \& Carter, C. S. (2000). Dissociating the role of the dorsolateral prefrontal and anterior cingulate cortex in cognitive control. Science, 288(5472), 1835-1838. https://doi.org/10.1126/science.288.5472.1835.

MacLeod, C.M. (1991). Half a century of research on the Stroop effect: an integrative review. Psychological Bulletin, 109, 163-203. https:// doi.org/10.1037/0033-2909.109.2.163.

Maclin, E., Gratton, G., \& Fabiani, M. (2001). Visual spatial localization conflict: An fMRI study. Neuroreport, 12(16), 3633-3636. https:// doi.org/10.1097/00001756-200111160-00051.

Mansouri, F. A., Egner, T., \& Buckley, M. J. (2017). Monitoring demands for executive control: Shared functions between human and 
nonhuman primates. Trends in Neurosciences, 40(1), 15-27. https:// doi.org/10.1016/j.tins.2016.11.001.

Masaki, H., Murphy, T. I., Desjardins, J. A., \& Segalowitz, S. J. (2012). The error-related negativity associated with different strength of stimulus-response interference. Clinical Neurophysiology, 123, 689-699. https://doi.org/10.1016/j.clinph.2011.07.043.

Mayka, M. A., Corcos, D. M., Leurgans, S. E., \& Vaillancourt, D. E. (2006). Three-dimensional locations and boundaries of motor and premotor cortices as defined by functional brain imaging: A metaanalysis. Neuroimage, 31(4), 1453-1474. https://doi.org/10.1016/j. neuroimage.2006.02.004.

Mayr, U., Awh, E., \& Laurey, P. (2003). Conflict adaptation effects in the absence of cognitive control. Nature Neuroscience, 6(5), 450-452. https://doi.org/10.1038/nn1051.

McCarthy, G., \& Donchin, E. (1981). A metric for though: A comparison of P300 latency and reaction time. Science, 211, 77-80. https://doi. org/10.1126/science.7444452.

Meckler, C., Allain, S., Carbonnell, L., Hasbroucq, T., Burle, B., \& Vidal, F. (2010). Motor inhibition and response expectancy: A Laplacian ERP study. Biological Psychology, 85(3), 386-392. https://doi.org/ 10.1016/j.biopsycho.2010.08.011.

Melara, R. D., Wang, H., Vu, K. P. L., \& Proctor, R. W. (2008). Attentional origins of the Simon effect: Behavioral and electrophysiological evidence. Brain Research, 1215, 147-159. https://doi.org/ 10.1016/j.brainres.2008.03.026.

Meynier, C., Burle, B., Possamaï, C.A., Vidal, F., \& Hasbroucq, T. (2009). Neural inhibition and interhemispheric connections in twochoice reaction time: a Laplacian ERP study. Psychophysiology, 46(4), 726-730. https://doi.org/10.1111/j.1469-8986.2009.00818.x.

Miller, E. K. (2000). The prefrontal cortex and cognitive control. Nature Reviews Neuroscience, 1(1), 59-65. https://doi.org/10.1038/ 35036228 .

Milner, A. D., \& Goodale, M. A. (1995). The Visual Brain in Action. Oxford (UK): Oxford University Press.

Miniussi, C., Harris, J. A., \& Ruzzoli, M. (2013). Modelling non-invasive brain stimulation in cognitive neuroscience. Neuroscience \& Biobehavioral Reviews, 37(8), 1702-1712. https://doi.org/10.1016/ j.neubiorev.2013.06.014

Missonnier, P., Deiber, M. P., Gold, G., Millet, P., Pun, M. G. F., FazioCosta, L., Giannakopoulus, P., Ibáñez, V. (2006). Frontal theta event-related synchronization: comparison of directed attention and working memory load effects. Journal of Neural Transmission, 113(10), 1477-1486.conflicts. Psychophysiology, 52(4), 562-571. https://doi.org/10.1007/s00702-005-0443-9.

Molenberghs, P., Mesulam, M. M., Peeters, R., \& Vandenberghe, R. R. C. (2007). Remapping attentional priorities: Differential contribution of superior parietal lobule and intraparietal sulcus. Cerebral Cortex, 17(11), 2703-2712. https://doi.org/10.1093/cercor/bhl179.

Müller, V., Langner, R., Cieslik, E., Rottschy, C., \& Eickhoff, S. (2015). Interindividual differences in cognitive flexibility: Influence of gray matter volume, functional connectivity and trait impulsivity. Brain Structure and Function, 220(4), 2401-2414. https://doi.org/10.1007/ s00429-014-0797-6.

Nachev, P., Husain, M., \& Kennard, C. (2008). Functional role of the supplementary and pre-supplementary motor areas. Nature Reviews Neuroscience, 9(11), 856-869. https://doi.org/10.1038/nrn2478.

Nee, D., Wager, T., \& Jonides, J. (2007). Interference resolution: Insights from a meta-analysis of neuroimaging tasks. Cognitive, Affective, \& Behavioral Neuroscience, 7(1), 1-17. https://doi.org/10.3758/ CABN.7.1.1.

Nicholson, R., Karayanidis, F., Poboka, D., Heathcote, A., \& Michie, P.T. (2005). Electrophysiological correlates of anticipatory taskswitching processes. Psychophysiology, 42(5), 540-554. https:// doi.org/10.1111/j.1469-8986.2005.00350.x.
Nicoletti, R., \& Umiltà, C. (1994). Attentional shifts produce spatial stimulus codes. Psychological Research, 56(3), 144-150. https:// doi.org/10.1007/BF00419701.

Nieuwenhuis, S., Stins, J. F., Posthuma, D., Polderman, T. J. C., Boomsma, D. I., \& de Geus, E. J. (2006). Accounting for sequential effects in the flanker task: conflict adaptation or associative priming? Memory \& Cognition, 34, 1260-1272. https://doi.org/10.3758/ BF03193270.

Nieuwenhuis, S., Yeung, N., Holroyd, C. B., Schurger, A., \& Cohen, J. D. (2004). Sensitivity of electrophysiological activity from medial frontal cortex to utilitarian and performance feedback. Cerebral Cortex, 14(7), 741-747. https://doi.org/10.1093/cercor/bhh034.

Nigbur, R., Ivanova, G., \& Stürmer, B. (2011). Theta power as a marker for cognitive interference. Clinical Neurophysiology, 122(11), 2185-2194. https://doi.org/10.1016/j.clinph.2011.03.030.

Notebaert, W., Soetens, E. \& Melis, A. (2001). Sequential analysis of a Simon task. Evidence for an attention-shift account. Psychological Research, 65(3), 170-184. https://doi.org/10.1007/s004260000054.

Nyberg, L. (2018). Cognitive control in the prefrontal cortex: A central or distributed executive? Scandinavian Journal of Psychology, 59(1), 62-65. https://doi.org/10.1111/sjop.12409.

O’Craven, K. M., Rosen, B. R., Kwong, K. K., Treisman, A., \& Savoy, R. L. (1997). Voluntary attention modulates fMRI activity in human MT-MST. Neuron, 18(4), 591-598. https://doi.org/10.1016/S08966273(00)80300-1.

O’Leary, M. J., \& Barber, P. J. (1993). Interference effects in the Stroop and Simon paradigms. Journal of Experimental Psychology: Human Perception and Performance, 19, 830-844. https://doi.org/ 10.1037/0096-1523.19.4.830.

O'Shea, J., Sebastian, C., Boorman, E. D., Johansen-Berg, H., \& Rushworth, M. F. S. (2007). Functional specificity of human premotor-motor cortical interactions during action selection. European Journal of Neuroscience, 26, 2085-2095. https://doi.org/ 10.1111/j.1460-9568.2007.05795.x.

Olk, B., Peschke, C., \& Hilgetag, C. C. (2015). Attention and control of manual responses in cognitive conflict: findings from TMS perturbation studies. Neuropsychologia, 74, 7-20. https://doi.org/10.1016/ j.neuropsychologia.2015.02.008.

Oostenveld, R., Praamstra, P., Stegeman, D. F., Van Oosterom, A. (2001). Overlap of attention and movement-related activity in lateralized event-related brain potentials. Clinical Neurophysiology, 112, 477-484. https://doi.org/10.1016/S13882457(01)00460-6.

Ouyang, G., Herzmann, G., Zhou, C., \& Sommer, W. (2011). Residue iteration decomposition (RIDE): A new method to separate ERP components on the basis of latency variability in single trials. Psychophysiology, 48, 1631-1647. https://doi.org/10.1111/j.14698986.2011.01269.x.

Page, L. A., Rubia, K., Deeley, Q., Daly, E., Toal, F., Mataix-Cols, D., ... Murphy, D.G. (2009). A functional magnetic resonance imaging study of inhibitory control in obsessive-compulsive disorder. Psychiatry Research: Neuroimaging, 174(3), 202-209. https://doi. org/10.1016/j.pscychresns.2009.05.002.

Pastötter, B., Dreisbach, G., \& Bäuml K. H. (2013). Dynamic adjustments of cognitive control: oscillatory correlates of the conflict adaptation effect. Journal of Cognitive Neuroscience, 25(12), 21672178. https://doi.org/10.1162/jocn_a_00474.

Pellicano, A., Lugli, L., Baroni, G., \& Nicoletti, R. (2009). The Simon effect with conventional signals. Experimental Psychology, 56 (4), 219-227. https://doi.org/10.1027/1618-3169.56.4.219.

Peschke, C., Köster, R., Korsch, M., Frühholz, S., Thiel, C. M., Herrmann, M., \& Hilgetag, C. C. (2016). Selective perturbation of cognitive conflict in the human brain-A combined fMRI and rTMS study. Scientific Reports, 6, 38700. https://doi.org/10.1038/ srep38700. 
Praamstra, P. (2006). Prior information of stimulus location: Effects on ERP measures of visual selection and response selection. Brain Research, 1072, 153-160. https://doi.org/10.1016/j.brainres.2005. 11.098.

Praamstra, P. (2007). Do's and don'ts with lateralized event-related brain potentials. Journal of Experimental Psychology: Human Perception and Performance, 33(2), 497-502. https://doi.org/10.1037/00961523.33.2.497.

Praamstra, P., Kleine, B. U., \& Schnitzler, A. (1999). Magnetic stimulation of the dorsal premotor cortex modulates the Simon effect. Neuroreport, 10, 3671-3674. https://doi.org/10.1097/00001756199911260-00038.

Praamstra, P., \& Oostenveld, R. (2003). Attention and movement-related motor cortex activation: a high-density EEG study of spatial stimulus-response compatibility. Cognitive Brain Research, 16(3), 309-322. https://doi.org/10.1016/S0926-6410(02)00286-0.

Proctor, R. W., Miles, J. D., \& Baroni, G. (2011). Reaction time distribution analysis of spatial correspondence effects. Psychonomic Bulletin \& Review, 18 (2), 242-266. https://doi.org/10.3758/ s13423-011-0053-5.

Proctor, R. W., \& Vu, K. P. L. (2006). Stimulus-response compatibility principles: Data, theory and application. Boca Raton: CRC Press.

Proctor, R. W., Vu, K.-P. L., \& Nicoletti, R. (2003). Does right-left prevalence occur for the Simon effect? Perception \& Psychophysics, 65, 1318-1329. doi:https://doi.org/10.3758/ BF03194855.

Provost, J., Hanganu, A., \& Monchi, O. (2015). Neuroimaging studies of the striatum in cognition part I: Healthy individuals. Frontiers in Systems Neuroscience, 9, 140. https://doi.org/10.3389/fnsys.2015. 00140.

Ragot, R. (1984). Perceptual and motor space representation: an eventrelated potential study. Psychophysiology, 21, 159-170. https://doi. org/10.1111/j.1469-8986.1984.tb00199.x.

Ragot, R., \& Remond, A. (1979). Event-related scalp potentials during a bimanual choice R.T. task. In E. Callaway \& R. Lehmann (Eds.). Human evoked potentials: Applications and problems (pp. 303316). Plenum Press, New York.

Ragot, R., \& Renault, B. (1981). P300 as a function of S-R compatibility and motor programming. Biological Psychology, 13, 289-294. https://doi.org/10.1016/0301-0511(81)90044-2.

Ridderinkhof, K. R. (2002). Activation and suppression in conflict tasks: Empirical clarification through distributional analyses. In W. Prinz \& B. Hommel (Eds.), Common mechanisms in perception and action. Attention \& performance (pp. 494-519). Oxford (UK): Oxford University Press

Ritter, W., Simson, R., \& Vaughan, H. G. (1972). Association cortex potentials and reaction time in auditory discrimination. Electroencephalography and Clinical Neurophysiology, 33(6), 547-555. https://doi.org/10.1016/0013-4694(72)90245-3.

Rizzolatti, G., Riggio, L., Dascola, I., \& Umiltà, C. (1987). Reorienting of attention across the horizontal and vertical meridians: Evidence in favor of a premotor theory of attention. Neuropsychologia, 25, 31 -40. https://doi.org/10.1016/0028-3932(87)90041-8.

Rosen, A. C., Rao, S. M., Caffarra, P., Scaglioni, A., Bobholz, J. A., Woodley, S. J., ... Binder, J. R. (1999). Neural basis of endogenous and exogenous spatial orienting: A functional MRI study. Journal of Cognitive Neuroscience, 11(2), 135-152. https://doi.org/10.1162/ 089892999563283

Rushworth, M. F., Krams, M., \& Passingham, R. E. (2001). The attentional role of the left parietal cortex: the distinct lateralization and localization of motor attention in the human brain. Journal of Cognitive Neuroscience, 13(5), 698-710. https://doi.org/10.1162/ 089892901750363244

Rushworth, M. F. S., Walton, M. E., Kennerley, S. W., \& Bannerman, D. M. (2004). Action sets and decisions in the medial frontal cortex.
Trends in Cognitive Sciences, 8(9), 410-417. https://doi.org/10. 1016/j.tics.2004.07.009.

Sato, W., Kochiyama, T., Uono, S., \& Toichi, M. (2016). Neural mechanisms underlying conscious and unconscious attentional shifts triggered by eye Ggaze. Neuroimage, 124, 118-126. https://doi.org/10. 1016/j.neuroimage.2015.08.061.

Schankin, A., Valle-Inclán, F., \& Hackley, S. A. (2010). Compatibility between stimulated eye, target location and response location. Psychological Research, 74(3), 291-301. https://doi.org/10.1007/ s00426-009-0247-x.

Scheeringa, R., Bastiaansen, M. C., Petersson, K. M., Oostenveld, R., Norris, D. G., \& Hagoort, P. (2008). Frontal theta EEG activity correlates negatively with the default mode network in resting state. International Journal of Psychophysiology, 67(3), 242-251. https:// doi.org/10.1016/j.ijpsycho.2007.05.017.

Schiff, S., Bardi, L., Basso, D., \& Mapelli, D. (2011). Timing spatial conflict within the parietal cortex: a TMS study. Journal of Cognitive Neuroscience, 23, 3998-4007. https://doi.org/10.1162/ jocn_a_00080.

Schlutter, N. D., Rushworth, M. F., Passingham, R. E., \& Mills, K. R. (1998). Temporary interference in human lateral premotor cortex suggests dominance for the selection of movements. A study using transcranial magnetic stimulation. Brain, 121, 785-799. https://doi. org/10.1093/brain/121.5.785.

Schmidt, J. R., Notebaert, W., \& Van Den Bussche, E. (2015). Is conflict adaptation an illusion? Frontiers in Psychology, 6:172. https://doi. org/10.3389/fpsyg.2015.00172.

Schmitz, N., Rubia, K., Daly, E., Smith, A., Williams, S., \& Murphy, D. G. M. (2006). Neural correlates of executive function in autistic spectrum disorders. Biological Psychiatry, 59(1), 7-16. https://doi. org/10.1016/j.biopsych.2005.06.007.

Sebastian, A., Gerdes, B., Feige, B., Klöppel, S., Lange, T., Philipsen, A., ... Tüscher, O. (2012). Neural correlates of interference inhibition, action withholding and action cancelation in adult ADHD. Psychiatry Research, 202(2), 132-141. https://doi.org/10.1016/j. pscychresns.2012.02.010.

Sebastian, A., Pohl, M.F., Klöppel, S., Feige, B., Lange, T., Stahl, C. ... Tüscher, O. (2013). Disentangling common and specific neural subprocesses of response inhibition. Neuroimage, 64, 601-615. https:// doi.org/10.1016/j.neuroimage.2012.09.020.

Sereno, M. I., \& Huang, R. S. (2014). Multisensory maps in parietal cortex. Current Opinion in Neurobiology, 24(1), 39-46. https://doi. org/10.1016/j.conb.2013.08.014.

Servant, M., White, C., Montagnini, A., \& Burle, B. (2016). Linking theoretical decision-making mechanisms in the Simon task with electrophysiological data: a model-based neuroscience study in humans. Journal of Cognitive Neuroscience, 28(10), 1501-1521. https://doi.org/10.1162/jocn_a_00989.

Shang, Q., Fu, H., Qiu, W., \& Ma, Q. (2016). Event-related lateralized readiness potential correlates of the emotion-priming Simon effect. Experimental Brain Research, 234(8), 2123-2132. https://doi.org/ 10.1007/s00221-016-4614-8.

Sheliga, B. M., Craighero, L., Riggio, L., \& Rizzolatti, G. (1997). Effects of spatial attention on directional manual and ocular responses. Experimental Brain Research, 114(2), 339-351. https://doi.org/10. 1007/PL00005642.

Simon, J. R., \& Small, A. M. (1969). Processing auditory information: interference from an irrelevant cue. Journal of Applied Psychology, 53, 433-435. https://doi.org/10.1037/h0028034.

Smulders, E. T. Y. (1993). The selectivity of age effects on information processing: response times and electrophysiology. Amsterdam: Universiteit van Amsterdam.

Smulders, F. T. Y., \& Miller, J. O. (2012). The lateralized readiness potential. In E. S. Kappenman, \& S. J. Luck (Eds.), The Oxford handbook of event-related potential components pp. 209-229. Oxford University Press. 
Sokolov, E. N. (1963). Perception and the conditioned reflex. Oxford: Pergamon Press.

Sommer, M., Hajak, G., Döhnel, K., Meinhardt, J., \& Müller, J. L. (2008). Emotion-dependent modulation of interference processes: An fMRI study. Acta Neurobiologiae Experimentalis Journal, 68(2), 193-203.

Sommer, W., Leuthold, H., \& Hermanutz, M. (1993). Covert effects of alcohol revealed by event-related potentials. Perception \& Psychophysics, 54, 127-135. https://doi.org/10.3758/BF03206944.

Spapé, M., \& Hommel, B. (2008). He said, she said: Episodic retrieval induces conflict adaptation in an auditory Stroop task. Psychonomic Bulletin \& Review, 15, 1117-1121.

Spapè, M. M., Band, G. P. H., \& Hommel, B. (2011). Compatibilitysequence effects in the Simon task reflect episodic retrieval but not conflict adaptation: evidence from LRP and N2. Biological Psychology, 88, 116-123. https://doi.org/10.1016/j.biopsycho. 2011.07.001.

Spapè, M. M., \& Hommel, B. (2014). Sequential modulations of the Simon effect depend on episodic retrieval. Frontiers in Psychology, 5(855). https://doi.org/10.3389/fpsyg.2014.00855.

Stoet, G. (2017). Sex differences in the Simon task help to interpret sex differences in selective attention. Psychological Research, 81(3), 571-581. https://doi.org/10.1007/s00426-016-0763-4.

Stoffels, E. J., van der Molen, M. W., \& Keuss, P. J. G. (1989). An additive factors analysis of the effect(s) of location cues associated with auditory stimuli on stages of information processing. Acta Psychologica 70(2), 161-197. https://doi.org/10.1016/00016918(89)90019-X.

Strack, G., Kaufmann, C., Kehrer, S., Brandt, S., \& Stürmer, B. (2013). Anticipatory regulation of action control in a Simon task: behavioral, electrophysiological, and fMRI correlates. Frontiers in Psychology, 4(47). https://doi.org/10.3389/fpsyg.2013.00047.

Stroop, J. R. (1935). Studies of interference in serial verbal reactions. Journal of Experimental Psychology, 18, 643-662. https://doi.org/ 10.1037/h0054651.

Stürmer, B., \& Leuthold, H. (2003). Control over response priming in visuomotor processing: a lateralized event-related potential study. Experimental Brain Research, 153(1), 35-44. https://doi.org/10. 1007/s00221-003-1579-1.

Stürmer, B., Leuthold, H., Soetens, E., Schröter, H., \& Sommer, W. (2002). Control over location-based response activation in the Simon task: behavioral and electrophysiological evidence. Journal of Experimental Psychology: Human Perception and Performance, 28(6), 1345-1363. https://doi.org/10.1037/0096-1523.28.6.1345.

Stürmer, B., Ouyang, G., Zhou, C., Boldt, A., \& Sommer, W. (2013). Separating stimulus-driven and response-related LRP components with Residue Iteration Decomposition (RIDE). Psychophysiology, 50(1), 70-73. https://doi.org/10.1111/j.1469-8986.2012.01479.x.

Stürmer, B., Redlich, M., Irlbacher, K., \& Brandt, S. (2007). Executive control over response priming and conflict: a transcranial magnetic stimulation study. Experimental Brain Research, 183(3), 329-339. https://doi.org/10.1007/s00221-007-1053-6.

Stürmer, B., Siggelkow, S., Dengler, R., \& Leuthold, H. (2000). Response priming in the Simon paradigm. Experimental Brain Research, 135, 353-359. https://doi.org/10.1007/s002210000529.

Symes, E., Ellis, R., \& Tucker, M. (2005). Dissociating object-based and space-based affordances. Visual Cognition, 12, 1337-1361. https:// doi.org/10.1080/13506280444000445.

Szucs, D., Soltész, F., Bryce, D., \& Whitebread, D. (2009). Real-time tracking of motor response activation and response competition in a Stroop task in young children: a lateralized readiness potential study. Journal of Congitive Neuroscience, 21(11), 2195-2206. https://doi. org/10.1162/jocn.2009.21220.
Tagliabue, M., Zorzi, M., Umiltà, C., \& Bassignani, F. (2000). The role of long-term memory and short-term-memory links in the Simon effect. Journal of Experimental Psychology: Human Perception and Performance, 26, 648-670. https://doi.org/10.1037/0096-1523.26. 2.648 .

Tang, J., Critchley, H. D., Glaser, D. E., Dolan, R. J., \& Butterworth, B. (2006). Imaging informational conflict: A functional magnetic resonance imaging study of numerical stroop. Journal of Cognitive Neuroscience, 18(12), 2049-2062. https://doi.org/10.1162/jocn. 2006.18.12.2049.

Töllner, T., Wang, Y., Makeig, S., Müller, H. J., Jung, T. P., \& Gramann, K. (2017). Two independent frontal midline theta oscillations during conflict detection and adaptation in a Simon-type manual reaching task. Journal of Neuroscience, 37(9), 2504-2515. https://doi.org/10. 1523/JNEUROSCI.1752-16.2017.

Treccani, T., Cona, G., Milanese, N., \& Umiltà, C. (2018). Sequential modulation of (bottom-up) response activation and inhibition in a response conflict task: a single-pulse transcranial magnetic stimulation study. Psychological Research, 82(4), 771-786. https://doi.org/ 10.1007/s00426-017-0863-9.

Treue, S., \& Maunsell, J. H. (1996). Attentional modulation of visual motion processing in cortical areas MT and MST. Nature, 382(6591), 539-541. https://doi.org/10.1038/382539a0.

Ulrich, R., Schröter, H., Leuthold, H., \& Birngruber, T. (2015). Automatic and controlled stimulus processing in conflict tasks: Superimposed diffusion processes and delta functions. Cognitive Psychology, 78, 148-174. https://doi.org/10.1016/j.cogpsych.2015. 02.005 .

Umebayashi, K., \& Okita, T. (2010). An ERP investigation of task switching using a flanker paradigm. Brain Research, 1346, 165173. https://doi.org/10.1016/j.brainres.2010.05.050.

Usher, M., \& McClelland, J. L. (2001). The time course of perceptual choice: The leaky, competing accumulator model. Psychological Review, 108(3), 550-592. https://doi.org/10.1037//0033-295X.108. 3.550 .

Vainio, L., Ellis, R., \& Tucker, M. (2007). The role of visuospatial attention in action priming. Quarterly Journal of Experimental Psychology, 60, 241-261. https://doi.org/10.1080/ 17470210600625149.

Valle-Inclán, F. (1996). The locus of interference in the Simon effect: An ERP study. Biological Psychology, 43(2), 147-162. https://doi.org/ 10.1016/0301-0511(95)05181-3.

Valle-Inclán, F., Hackley, S. A., \& De Labra, C. (2003). Stimulusresponse compatibility between stimulated eye and response location: Implications for attentional accounts of the Simon effect. Psychological Research, 67, 240-243. https://doi.org/10.1007/ s00426-003-0131-z.

Valle-Inclán, F. \& Redondo, M. (1998). On the automaticity of ipsilateral response activation in the Simon effect. Psychophysiology, 35(4), 366-371. https://doi.org/10.1111/1469-8986.3540366.

Valle-Inclán, F., Sohn, F., \& Redondo, M. (2008). Spatial compatibility between stimulated eye and response location. Psychophysiology, 45, 279-285. https://doi.org/10.1111/j.1469-8986.2007.00616.x.

Vallesi, A., Mapelli, D., Schiff, S., Amodio, P., \& Umiltà, C. (2005). Horizontal and vertical Simon effect: different underlying mechanism? Cognition 96, 33-43. https://doi.org/10.1016/j.cognition. 2004.11.009.

Van Campen, A. D., Keuken, M. C., van den Wildenberg, W. P., \& Ridderinkhof, K. R. (2014). TMS over M1 reveals expression and selective suppression of conflicting action impulses. Journal of Cognitive Neuroscience, 26, 1-15. https://doi.org/10.1162/jocn_a 00482. 
van Campen, A. D., Kunert R., van den Wildenberg W. P. M., Ridderinkhof, K. R. (2018). Repetitive transcranial magnetic stimulation over inferior frontal cortex impairs the suppression (but not expression) of action impulses during action conflict. Psychophysiology. 55(3), 1-13. https://doi.org/10.1111/psyp.13003.

Van der Lubbe, R. H. J., Abrahamse, E. L., \& De Kleine, E. (2012). The premotor theory of attention as an account for the Simon effect. Acta Psychologica, 140(1), 25-34. https://doi.org/10.1016/j.actpsy.2012. 01.011 .

Van der Lubbe, R. H. J., Jaskowśki, P., \& Veleger, R. (2005). Mechanisms underlying spatial coding in a multiple-item Simon task. Psychological Research, 69, 179-190. https://doi.org/10. 1007/s00426-004-0176-7.

van Driel, J., Ridderinkhof, K. R., \& Cohen, M. X. (2012). Not all errors are alike: theta and alpha EEG dynamics relate to differences in error-processing dynamics. Journal of Neuroscience, 32(47), 16795-16806. https://doi.org/10.1523/JNEUROSCI.0802-12.2012.

van Driel, J., Swart, J. C., Egner, T., Ridderinkhof, K. R., \& Cohen, M. X. (2015). (No) time for control: frontal theta dynamics reveal the cost of temporally guided conflict anticipation. Cognitive, Affective, \& Behavioral Neuroscience, 15(4), 787-807. https://doi.org/10.3758/ s13415-015-0367-2.

van Eijk, J., Sebastian, A., Krause-Utz, A., Cackowski, S., Demirakca, T., Biedermann, S.V., ... Tüscher, O. (2015). Women with borderline personality disorder do not show altered BOLD responses during response inhibition. Psychiatry Research: Neuroimaging, 234(3), 378-389. https://doi.org/10.1016/j.pscychresns.2015.09.017.

van Gaal, S., Lamme, V. A. F., \& Ridderinkhof, K. R. (2010). Unconsciously triggered conflict adaptation. PLoS One, 5, e11508. https://doi.org/10.1371/journal.pone.0011508.

Van Veen, V., \& Carter, C. S. (2002). The timing of action-monitoring processes in the anterior cingulate cortex. Journal of Cognitive Neuroscience, 14, 593-602. https://doi.org/10.1162/ 08989290260045837.

Verleger, R. (1997). On the utility of P3 latency as an index of mental chronometry. Psychophysiology, 34, 131-156. https://doi.org/10. 1111/j.1469-8986.1997.tb02125.x.

Verleger, R., Jaśkowski, P., \& Wascher, E. (2005). Evidence for an integrative role of $\mathrm{P} 3 \mathrm{~b}$ in linking reaction to perception. Journal of Psychophysiology, 19, 165-181. https://doi.org/10.1027/02698803.19.3.165.

Verleger, R., Kuniecki, M., Möller, F., Fritzmannova, M., \& Siebner, H. R. (2009). On how the motor cortices resolve an inter-hemispheric response conflict: an event-related EEG potential-guided TMS study of the flankers task. European Journal of Neuroscience, 30(2), 318 326. https://doi.org/10.1111/j.1460-9568.2009.06817.x.

Vidal, F., Burle, B., Grapperon, J., \& Hasbroucq, T. (2011). An ERP study of cognitive architecture and the insertion of mental processes: Donders revisited. Psychophysiology, 48(9), 1242-1251. https://doi. org/10.1111/j.1469-8986.2011.01186.x.

Vissers, M. E., Ridderinkhof, K. R., Cohen, M. X., \& Slagter, H. A. (2018). Oscillatory mechanisms of response conflict elicited by color and motion direction: An individual differences approach. Journal of Cognitive Neuroscience, 30(4), 468-481. https://doi.org/10.1162/ jocn a 01222 .

Wang, K., Li, Q., Zheng, Y., Wang, H., \& Liu, X. (2014). Temporal and spectral profiles of stimulus-stimulus and stimulus-response conflict processing. Neuroimage, 89, 280-288. https://doi.org/10.1016/ j.neuroimage.2013.11.045.

Wascher, E. (2005). The timing of stimulus localisation and the Simon effect: an ERP study. Experimental Brain Research 163(4), 430439. https://doi.org/10.1007/s00221-004-2198-1.
Wascher, E., Schatz, U., Kuder, T., \& Verleger, R. (2001). Validity and boundary conditions of automatic response activation in the Simon task. Journal of Experimental Psychology: Human Perception and Performance, 27, 731-751. https://doi.org/10.1037//0096-1523.27. 3.731.

Wascher, E., \& Wauschkuhn, B. (1996). The interaction of stimulus- and response-related processes measured by event-related lateralizations of the EEG. Electroencephalography and Clinical Neurophysiology, 99, 149-162. https://doi.org/10.1016/0013-4694(96)95602-3.

Weissman, D. H., Jiang, J., \& Egner, T. (2014). Determinants of congruency sequence effects without learning and memory confounds. Journal of Experimental Psychology: Human Perception and Performance, 40(5), 2022-2037. https://doi.org/10.1037/a0037454.

Wendelken, C., Ditterich, J., Bunge, S., \& Carter, C. (2009). Stimulus and response conflict processing during perceptual decision making. Cognitive, Affective, \& Behavioral Neuroscience, 9(4), 434-447. https://doi.org/10.3758/CABN.9.4.434.

Wendt, M., Heldmann, M., Münte, T. F., \& Kluwe, R. H. (2007) Disentangling sequential effects of stimulus- and response-related conflict and stimulus-response repetition using brain potentials. Journal of Cognitive Neuroscience, 19(7), 1104-1112. https://doi. org/10.1162/jocn.2007.19.7.1104.

Weston, C. S. E. (2012). Another major function of the anterior cingulate cortex: The representation of requirements. Neuroscience \& Biobehavioral Reviews, 36(1), 90-110. https://doi.org/10.1016/j. neubiorev.2011.04.014.

Wiegand, K., \& Wascher, E. (2005). Dynamic aspects of stimulusresponse correspondence: evidence for two mechanisms involved in the Simon effect. Journal of Experimental Psychology: Human Perception and Performance, 31, 453-464. doi:https://doi.org/10. 1037/0096-1523.31.3.453.

Wild-Wall, N., Falkenstein, M., \& Hohnsbein, J. (2008). Flanker interference in young and older participants as reflected in event-related potentials. Brain Research, 1211, 72-84. https://doi.org/10.1016/j. brainres.2008.03.025.

Wittfoth, M., Buck, D., Fahle, M., \& Herrmann, M. (2006). Comparison of two Simon tasks: Neuronal correlates of conflict resolution based on coherent motion perception. Neuroimage, 32(2), 921-929. https://doi.org/10.1016/j.neuroimage.2006.03.034.

Wittfoth, M., Schardt, D. M., Fahle, M., \& Herrmann, M. (2009). How the brain resolves high conflict situations: Double conflict involvement of dorsolateral prefrontal cortex. Neuroimage, 44(3), 12011209. https://doi.org/10.1016/j.neuroimage.2008.09.026.

Woodman, G. F., \& Luck, S. J. (1999). Electrophysiological measurement of rapid shifts of attention during visual search. Nature, 400(6747), 867-869. https://doi.org/10.1038/23698.

Wühr, P. (2005). Evidence for gating of direct response activation in the Simon task. Psychonomic Bulletin \& Review, 12, 282-288. https:// doi.org/10.3758/BF03196373.

Xia, T., Li, H., \& Wang, L. (2016). Implicitly strengthened task-irrelevant stimulus-response associations modulate cognitive control: Evidence from an fMRI study. Human Brain Mapping, 37(2), 756-772. https://doi.org/10.1002/hbm.23064.

Xu, M., Xu, G., \& Yang, Y. (2016). Neural systems underlying emotional and non-emotional interference processing: An ALE meta-analysis of functional neuroimaging studies. Frontiers in Behavioral Neuroscience, 10, 220. https://doi.org/10.3389/fnbeh.2016.00220.

Yantis, S., Schwarzbach, J., Serences, J. T., Carlson, R. L., Steinmetz, M. A., Pekar, J. J., \& Courtney, S. M. (2002). Transient neural activity in human parietal cortex during spatial attention shifts. Nature Neuroscience, 5(10), 995-1002. https://doi.org/10.1038/nn921.

Zhang, R., Geng, X., \& Lee, T. (2017). Large-scale functional neural network correlates of response inhibition: An fMRI meta-analysis. 
Brain Structure and Function, 222(9), 3973-3990. https://doi.org/ 10.1007/s00429-017-1443-x.

Zorzi, M., \& Umiltà, C. (1995). A computational model of the Simon effect. Psychological Research, 58(3), 193-205. https://doi.org/10. 1007/BF00419634

Zurrón, M., Lindín, M., Cespón, J., Cid-Fernández, S., Galdo-Álvarez, S., Ramos-Goicoa, M., \& Díaz, F. (2018). Effects of mild cognitive impairment on the event-related brain potential components elicited in executive control tasks. Frontiers in Psychology, 9, 842. https:// doi.org/10.3389/fpsyg.2018.00842.

Publisher's note Springer Nature remains neutral with regard to jurisdictional claims in published maps and institutional affiliations. 